\title{
TASI Lectures on Early Universe Cosmology: Inflation, Baryogenesis and Dark Matter
}

\author{
James M. Cline* \\ Dept. of Physics, McGill University, Montréal, Québec, Canada \\ E-mail: jcline@physics.mcgill.ca
}

\begin{abstract}
These lectures, presented at TASI 2018, provide a concise introduction to inflation, baryogenesis, and aspects of dark matter not covered by the other lectures. The emphasis for inflation is an intuitive understanding and techniques for constraining inflationary models. For baryogenesis we focus on two examples, leptogenesis and electroweak baryogenesis, with attention to singletassisted two-step phase transitions. Concerning dark matter, we review different classes of models distinguished by their mechanisms for obtaining the observed relic density, including thermal freeze-out, asymmetric dark matter, freeze-in, SIMP dark matter, the misalignment mechanism for ultralight scalars and axions, and production of primordial black holes during inflation. Problem sets are provided.
\end{abstract}

Theoretical Advanced Study Institute Summer School 2018 'Theory in an Era of Data'(TASI2018) 4 - 29 June, 2018

Boulder, Colorado

${ }^{*}$ Speaker. 


\section{Introduction}

For these lectures I was assigned the topic of "Early Universe Cosmology." If we could go back in time fifty years, this would seem like a more straightforward task, since the cosmological timeline was relatively uncrowded by notable events; see fig. 1. Following the big bang, there was nucleosynthesis (BBN), matter-radiation equality, recombination, and formation of galactic structure. But our current understanding intersperses many more likely or at least possible events of significance (fig. 2), replacing the big bang by inflation, introducing leptogenesis or baryogenesis, several cosmological phase transitions, and some kind of origin for the dark matter (DM) of the universe. For these lectures I have therefore chosen to discuss inflation, baryogenesis, and aspects of dark matter not covered by other lecturers. P. Fox has included a nice introduction to BBN as well as thermal freeze-out of dark matter in his lectures on SUSY WIMPs, while direct and indirect detection of DM are treated respectively by T. Lin and D. Hooper, and axions by A. Hook. Structure formation will be introduced by M. Vogelsberger. Hence I hope that the more general picture of early universe cosmology will get a comprehensive treatment through our combined lectures.

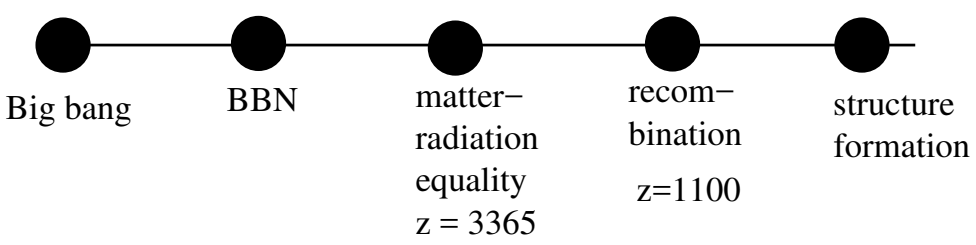

Figure 1: The cosmological timeline, ca. 1970.

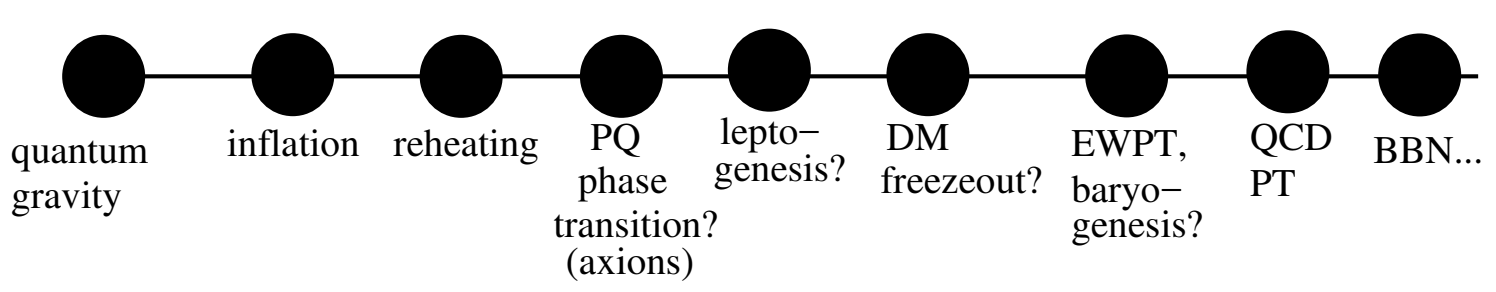

Figure 2: The cosmological timeline, ca. 2018.

\subsection{Conventions and basics of big bang cosmology}

I will be using natural units,

$$
\hbar=c=k_{B}=1, \quad G=\frac{1}{M_{p}^{2}}, \quad 8 \pi G=\frac{1}{m_{p}^{2}}
$$

The reader should be warned that my choice of upper and lower case for the unreduced Planck mass $M_{p}=1.22 \times 10^{19} \mathrm{GeV}$ and the reduced one, $m_{p}=2.43 \times 10^{18} \mathrm{GeV}$, is not a standard convention, even though it seems logical. The Einstein equations then read

$$
G_{\mu \nu}=\frac{1}{m_{p}^{2}} T_{\mu \nu} \quad \longleftrightarrow \quad R_{\mu \nu}=\frac{1}{m_{p}^{2}}\left(T_{\mu \nu}-\frac{1}{2} g_{\mu \nu} T\right)
$$


with

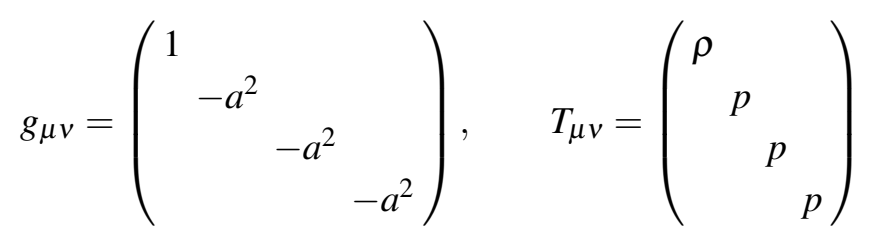

Basic elements of cosmology are summarized in the PDG reviews [1] or the textbook of Kolb and Turner [2]; I recapitulate them here for convenience. The Friedmann-Robertson-Walker (FRW) line element is

$$
d s^{2}=d t^{2}-a^{2}(t) d x^{2}
$$

where $d x^{2}$ represents a unit 3D metric that can have curvature $K=0, \pm 1$. Redshift is defined by $1+z=a_{0} / a$, where $a_{0}$ is the present value of the scale factor, while the Hubble parameter is

$$
H=\frac{\dot{a}}{a} \quad\left(\dot{H}=\frac{\ddot{a}}{a}-H^{2}\right)
$$

Then the (00) and $(i j)$ components of the Einstein equations can be written respectively as

$$
H^{2}=\frac{\rho}{3 m_{p}^{2}}-\frac{k}{a^{2}} 2 \frac{\ddot{a}}{a}+H^{2}=-\frac{p}{m_{p}^{2}}-\frac{k}{a^{2}},
$$

where $k$ has units of $1 /(\text { distance })^{2}$ if $a$ is taken to be dimensionless. The first of these is the usual Friedmann equation that together with the equation of state fixing $\rho$ as a function of $a$ determines the evolution of a homogeneous universe. Although the present universe does not look very homogeneous at first glance, the approximation starts to be valid when averaging over scales $\gtrsim 70 \mathrm{Mpc}$ [3]. More importantly for these lectures, the cosmic microwave background (CMB) shows that the universe was very homogeneous, to a part in 20,000, at $z=1100$.

To complete the Friedmann equation we need the time- or $a$-dependence of the energy density $\rho$, which is determined by the equation of state (EOS),

$$
p=w \rho, \quad w=\left\{\begin{array}{c}
\frac{1}{3}, \text { radiation } \\
0, \text { matter } \\
-1, \text { vacuum energy }
\end{array}\right.
$$

The conservation of stress-energy, $\partial_{\mu} T^{\mu v}=0$, implies that $\dot{\rho}=-3 H(\rho+p)=-3 H \rho(1+w)$, and combining this with (1.8) gives

$$
\rho \sim\left\{\begin{array}{l}
1 / a^{4}, \text { radiation } \\
1 / a^{3}, \text { matter } \\
\text { const., vacuum energy }
\end{array}\right.
$$

Taking $a_{0}=1$, we can integrate the Friedmann equation,

$$
\int d t= \pm \sqrt{3} m_{p} \int d a\left(\frac{\rho_{r, 0}}{a^{2}}+\frac{\rho_{m, 0}}{a}+\rho_{\Lambda} a^{2}-\rho_{k}\right)^{-1 / 2}
$$

where $\rho_{k}$ is a fictitious energy density going as $K m_{p}^{2} / R_{0}^{2}$, with $K=0, \pm 1$ and $R_{0}$ being a physical length scale for the $3 \mathrm{D}$ curvature. $\rho_{x, 0}$ denotes the present density of matter $(x=m)$ or radiation 
$(x=r)$. Although eq. (1.10) cannot be usefully integrated in closed form in the general case, it is easy to do so when any of the individual terms dominate, in particular

$$
a \sim\left\{\begin{array}{c}
t^{1 / 2}, \text { radiation } \\
t^{2 / 3}, \text { matter } \\
\exp \left(t \sqrt{\rho_{\Lambda} / 3} / m_{p}\right), \text { vacuum energy }
\end{array}\right.
$$

Introducing the critical density $\rho_{c}=3 m_{p}^{2} H_{0}^{2} \cong(2.47 \mathrm{meV})^{4}$, we can define the fractional contributions of the various components to the total energy density of the universe,

$$
\Omega_{i}=\frac{\rho_{i}}{\rho_{c}} \cong\left\{\begin{array}{c}
5 \times 10^{-5}, \gamma \\
0.05, \text { baryons } \\
0.26, \mathrm{CDM} \\
0.69, \Lambda \\
<0.015, k \text { (curvature) }
\end{array}\right.
$$

which identically satisfy $\sum_{i} \Omega_{i}=1$ when including $\Omega_{k}$ in the sum. CDM stands for cold dark matter, and the upper limit on $\Omega_{k}$ applies (roughly) to its absolute value.

\section{Inflation}

Although the cosmological timeline looked simple in 1970, by later in the decade an awareness was building that all was not well with the big bang picture; see for example the essay by Dicke and Peebles in ref. [4]. This was due to the now famous horizon and flatness problems.

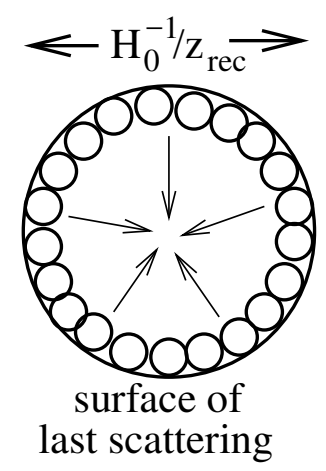

Figure 3: The horizon problem; we are at the center.

\subsection{Horizon problem}

CMB photons have been free-streaming from the surface of last scattering, representing the epoch when the universe became transparent, around the time of electron-proton recombination, fig. 3. To understand this picture we need the idea of the particle horizon $d_{H}(t)$, which is the distance that a photon could have traveled by a given time $t$. Since photons follow null worldlines, $d t=a d x$, we have

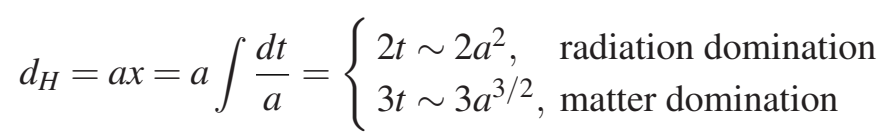


$d_{H}(t)$ is therefore the maximum size of a causally connected region at time $t$, within which one could expect any degree of uniformity due to thermal equilibration. Our current horizon is $d_{H}\left(t_{0}\right) \sim$ $H_{0}^{-1} \sim 10^{26} \mathrm{~m}$, and the CMB radiation contained within it is extremely uniform, to better than $10^{-4}$, suggesting that the region containing the whole presently observable universe was already in causal contact at the time of recombination $t_{\text {rec }}$. Yet if we consider how large this region was at that time, $\sim d_{H}\left(t_{0}\right) / z_{\text {rec }}$, by rescaling it according to the Hubble expansion, it is much larger than $d_{H}\left(t_{\text {rec }}\right)$. This is the conundrum illustrated in fig. 3. The small circles represent the largest regions that should have uniform temperature. We can estimate the number of them around the big circle as

$$
\frac{2 \pi d_{H}\left(t_{0}\right) / z_{\mathrm{rec}}}{2 d_{H}\left(t_{\mathrm{rec}}\right.}=\pi \sqrt{z_{\mathrm{rec}}} \cong 100
$$

so that each one subtends an angle of $\Delta \theta \cong 3.5^{\circ}$. How did the temperature come to be so uniform across all of these regions?

\subsection{Flatness problem}

The inverse curvature radius $R^{-1}$ must be tuned to an extremely small value in order for the $\rho_{k}$ term in (1.10) to avoid dominating the current expansion of the universe. Recalling the relation $\left|\rho_{k}\right|=3 m_{p}^{2} / R_{0}^{2}$, we see that

$$
\left|\Omega_{k}\right|=\left|\frac{\rho_{k}}{\rho_{c}}\right|=\frac{1}{H_{0}^{2} R_{0}^{2}} \quad \Rightarrow \quad R_{0} \gtrsim \frac{10}{H_{0}} \sim 10^{27} \mathrm{~m} \sim 30,000 \mathrm{Mpc}
$$

The curvature radius scales simply with the Hubble expansion. Scaling back to the Planck time $t_{p} \sim 1 / m_{p}$, we get

$$
R_{p}=R_{0} a_{p} \sim R_{0} \frac{T_{\gamma, 0}}{m_{p}} \sim \frac{10}{H_{0}} \frac{T_{\gamma}}{m_{p}} \sim \frac{10 T_{\gamma}}{\rho_{c}^{1 / 2}}=\frac{2.4 \times 10^{-3} \mathrm{eV}}{\left(2.5 \times 10^{-3} \mathrm{eV}\right)^{2}} \sim \frac{1}{3 \times 10^{-3} \mathrm{eV}}
$$

This is to be compared to the natural value $R_{p} \sim 1 / m_{p}$, since $m_{p}$ is the only relevant dimensionful parameter. Thus we see that a tuning of one part in $10^{31}$ is required for the initial curvature radius, if we are allowed to extrapolate back as far as $m_{p}$. Limiting the earliest time to larger values only gives a modest improvement unless we push that time all the way into the present.

\subsection{A little history}

In 1979, the hot topic was grand unified theories (GUTs). In the previous year, Zeldovich and Khlopov [5] had estimated that if the universe ever went through the GUT symmetry breaking transition, then pointlike topological defects, magnetic mononpoles, with masses of $M_{\mathrm{GUT}} \sim 10^{16} \mathrm{GeV}$ would have been so copiously produced that the universe should have recollapsed shortly thereafter. J. Preskill, at that time a graduate student at Harvard, corrected an important overestimate in their calculation but nevertheless confirmed their conclusion [6]. ${ }^{1}$ At that time A. Guth was a postdoc at Cornell, and with $\mathrm{H}$. Tye proposed some ideas for suppressing the density of monopoles [7]. He then moved to SLAC, taking up his fourth postdoctoral position, still thinking about the

\footnotetext{
${ }^{1}$ It required some conviction on his part, since his supervisor S. Weinberg reportedly told him he was "crazy" to work on that problem.
} 
monopole problem. It led him to propose inflation [8], which he recognized did much more than solve the monopole problem; it also solved the horizon and flatness problems. Soon thereafter, people realized that it additionally explained the origin of density perturbations leading to large scale structure.

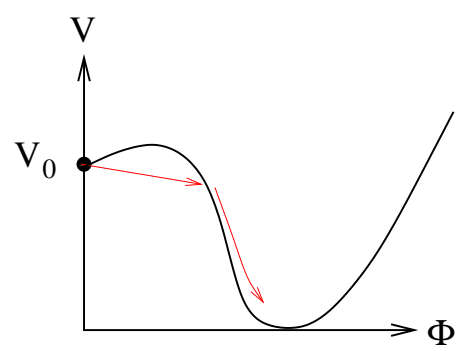

Figure 4: The SU(5) GUT potential near the critical temperature, leading to a first order phase transition.

Guth's original idea, now called "old inflation," was inspired by the SU(5) GUT, which requires a Higgs field $\varphi$ to break SU(5) down to the standard model gauge group. At high temperatures, thermal effects make $m_{\varphi}^{2}>0$ at the origin $\varphi=0$, but as $T$ decreases, a local minimum develops at $\varphi \neq 0$ as shown in fig. 4. Initially $\varphi$ is trapped in the false minimum, whose vacuum energy causes the universe to expand as

$$
a \sim \exp \left(t \sqrt{V_{0} / 3} / m_{p}\right)
$$

Inflation ends when $\varphi$ tunnels through the barrier and rolls down to the true minimum. But as Guth realized even in the seminal paper, this picture is flawed because the tunneling leads to nucleation of bubbles of true vacuum that are cold and empty. These universes could be heated up by the energy released by collisions of walls from neighboring bubbles, but first of all these collisions are exceedingly rare - the phase transition never completes-and second, the resulting radiation would be quite inhomogeneous, undoing all the smoothing of initial inhomogeneities that occurred during inflation. A further problem, seemingly not noticed at the time, is that bubbles nucleated in this way have very large negative curvature [9], undoing the solution of the flatness problem afforded by the initial inflationary expansion. However it was soon realized that the potential in the post-nucleation phase could be made sufficiently flat so that inflation would still take place, with no need for the prior false-vacuum phase [10,11], except perhaps to justify the initial condition by tunneling. This was dubbed "new inflation."

It is sometimes noted that the first model of inflation was published by Starobinsky [12] before all of these developments, based upon an $R^{2}$ addition to the Einstein-Hilbert gravitational action. This is particularly interesting now because of the preference given to this model for fitting current CMB data as observed by Planck [13]. However Starobinsky was not aware at that time that inflation was a general mechanism that could solve the problems of big bang cosmology as emphasized by Guth.

\subsection{Inflation in brief}

We now recapitulate the main features of inflation, settling for a heuristic rather than a rigorous approach. I will fill in some details in the following subsection. Inflation can be driven by any scalar 
field $\varphi$ whose potential is sufficiently flat, as measured by the potential slow roll parameters

$$
\varepsilon=\frac{m_{p}^{2}}{2}\left(\frac{V^{\prime}}{V}\right)^{2}, \quad \eta=m_{p}^{2} \frac{V^{\prime \prime}}{V}
$$

This parametrization is perhaps getting outdated since many practitioners prefer the Hubble flow functions

$$
\begin{aligned}
& \varepsilon_{1}=-\frac{\dot{H}}{H^{2}} \cong \varepsilon \\
& \varepsilon_{2}=\frac{\dot{\varepsilon}_{1}}{H \varepsilon_{1}} \cong 4 \varepsilon-2 \eta \\
& \varepsilon_{3}=\frac{\dot{\varepsilon}_{2}}{H \varepsilon_{2}} \ldots
\end{aligned}
$$

but for leading-order calculations (involving only $\varepsilon$ and $\eta$ ) either is sufficient. When $\varepsilon, \eta \ll 1$, we can ignore the $\ddot{\varphi}$ term in the equation of motion (EOM) for the homogeneous mode of $\varphi$, approximating it by the slow-roll EOM,

$$
3 H \dot{\varphi} \cong-V^{\prime}(\varphi)
$$

The Hubble damping term comes from varying the scalar field action in the presence of the background metric, $S=\int d^{4} x a^{3}\left(a^{-2} \dot{\varphi}^{2} / 2-V\right)$. The kinetic energy of $\varphi$ is then much smaller than $V$, and the Friedmann equation can be integrated to find

$$
a \sim \exp \left(\int H d t\right) \equiv e^{N}
$$

with $H=\sqrt{V / 3 m_{p}^{2}}$ and $N$ being the number of e-foldings. Eventually, as $\varphi$ reaches the minimum of its potential, either $\varepsilon$ or $\eta$ will exceed unity, and $\ddot{\varphi}$ can no longer be ignored; instead $H \dot{\varphi}$ becomes negligible,

$$
\ddot{\varphi} \cong-V^{\prime}(\varphi)
$$

and $\varphi$ oscillates around the minimum of $V$. These oscillations lead to particle production and reheating of the universe.

A naive estimate of the reheating temperature is

$$
T_{\mathrm{rh}} \sim \sqrt{m_{p} \Gamma_{\varphi}}
$$

where $\Gamma_{\varphi}$ is the decay rate of the inflaton. One can easily derive (2.11) from the usual relation between time and temperature,

$$
t \sim \frac{1}{\Gamma_{\varphi}} \sim \frac{1}{H} \sim \frac{m_{p}}{\sqrt{\rho}} \sim \frac{m_{p}}{T_{\mathrm{rh}}^{2}}
$$

which is valid as long as the computed $T_{\text {rh }}$ does not exceed the available energy scale from inflation, $V_{i}^{1 / 4}$ (where $V_{i}$ is the magnitude of $V$ during inflation). It may seem counterintuitive that $T_{\mathrm{rh}}$ would be independent of $V_{i}$, but this occurs because the particles produced by the early decays are diluted 
by continuing quasi-exponential expansion of the universe; only those produced near the end of the reheating phase dominate the final density. See problem 2 of the inflation exercises.

Let's review how inflation solves the problems of big bang cosmology. The flatness problem is solved by the stretching of space by the expansion, which increases an initial curvature radius $R_{i}$ as

$$
\frac{1}{R_{i}^{2}} \rightarrow \frac{e^{-2 N}}{R_{i}^{2}}
$$

To reduce $1 / R$ by a factor of $10^{31}$, as we motivated in our example, would require $N=70$ e-foldings of inflation. This is actually an overestimate since $1 / R_{i}$ must be somewhat smaller than $m_{p}$ in order for inflation to get started (at least if the curvature is positive): $H^{2}=V(\varphi) / 3 m_{p}^{2}-1 /\left(a^{2} R_{i}^{2}\right)$ must be positive. Then if $V_{i} \equiv \Lambda_{i}^{4}$, we require $1 / R_{i} \lesssim \Lambda_{i}^{2} / m_{p}$. Its value today would be

$$
\frac{1}{R_{0}} \sim \frac{e^{-N}}{R_{i}}\left(\frac{T_{0}}{T_{\mathrm{rh}}}\right)
$$

which requires

$$
N>\ln \frac{R_{0} T_{0}}{R_{i} T_{\mathrm{rh}}}>\ln \frac{10 H_{0}^{-1} T_{0} \Lambda_{i}^{2}}{m_{p} T_{\mathrm{rh}}} \sim \ln \frac{10 T_{0} \Lambda_{i}^{2}}{\rho_{c}^{1 / 2} T_{\mathrm{rh}}}
$$

Taking for example $\Lambda_{i}=10^{-3} m_{p}$ (we will see that CMB data provide this as an upper limit) and $T_{\text {rh }} \sim \Lambda_{i}$, we find

$$
N \gtrsim \ln \frac{10^{-3} \mathrm{eV} \times 10^{22} \mathrm{eV}}{10^{-6} \mathrm{eV}^{2}} \cong 58
$$

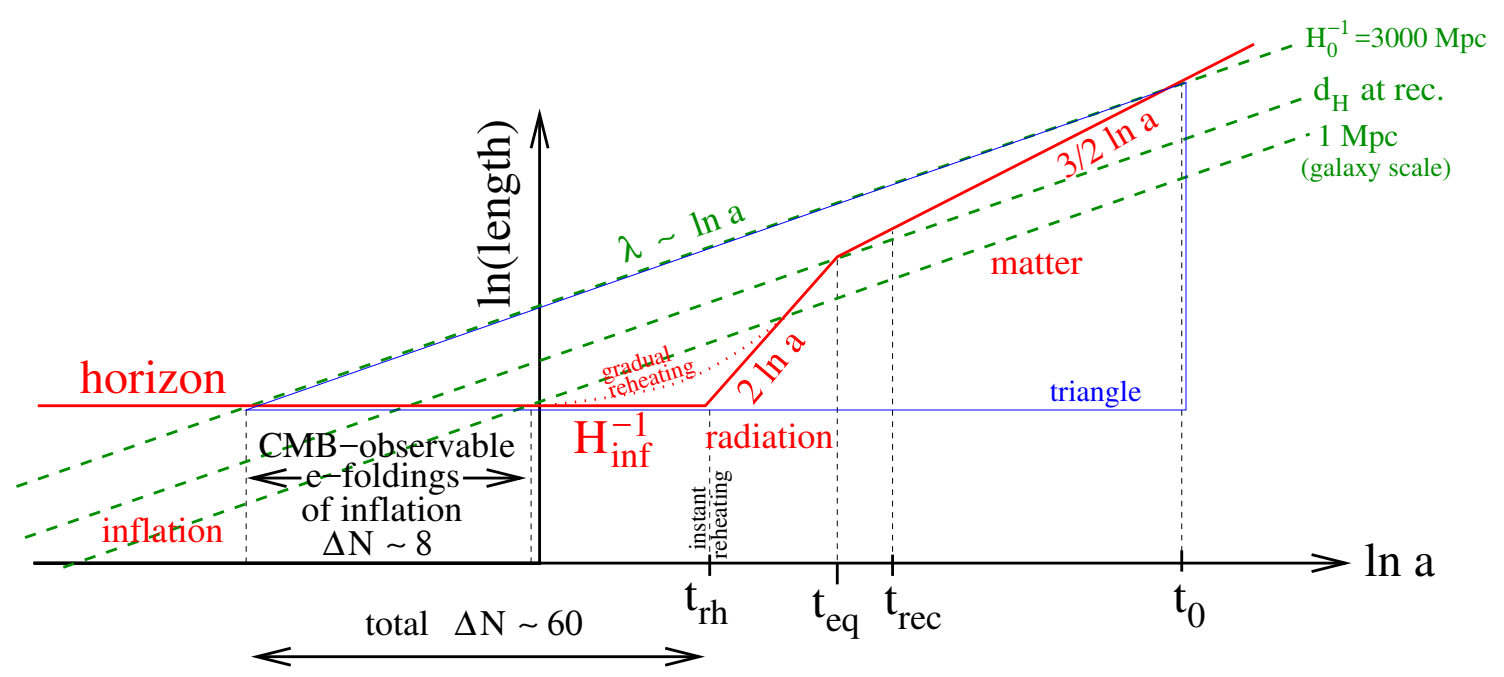

Figure 5: Evolution of comoving scales (green, dashed) and the particle horizon (red, solid) with scale factor as a proxy for time.

For the horizon problem, fig. 5 (inspired by fig. 8.4 of ref. [2]) is helpful. The dashed line labeled " $H_{0}^{-1}=3000 \mathrm{Mpc}$ " is relevant for the horizon problem: it shows how the current horizon scale shrinks with the universe projected back in time, being much larger than the maximum causally connected scale $d_{H}\left(t_{\text {rec }}\right)$ at the time of recombination. In standard big bang cosmology, 
the $H_{0}^{-1}$ scale was always outside the horizon at earlier times, signifying that its enclosed particles could not have achieved significant causal equilibrium. But with inflation, provided it lasted long enough, there is an intersection such that at early times it was inside the horizon and causal processes would have been able to make it homogeneous.

We can estimate the minimum number of $e$-foldings needed to solve the horizon problem using this figure and a bit of trigonometry. Consider the right triangle (blue, labeled "triangle") whose base extends along the inflationary horizon, from where the $H_{0}^{-1}$ scale acting as hypotenuse intersects it, until the present time. The vertical leg of the triangle has length $\ln \left(H_{\text {inf }} / H_{0}\right)$, while the horizontal one has height $\Delta N+\ln \left(T_{\mathrm{rh}} / T_{0}\right)$. The slope is unity since scales grow linearly with $a$. This gives the minimum number of $e$-foldings of inflation as

$$
\Delta N=\ln \frac{H_{\text {inf }} T_{0}}{H_{0} T_{\mathrm{rh}}}
$$

This is a rather crude approximation, since we have assumed in the picture that reheating happens instantaneously, which would imply a very efficient mechanism of reheating such that $T_{\mathrm{rh}} \sim \Lambda_{\text {inf }}$ (the energy scale of inflation). Nevertheless, we estimate $\Delta N \sim \ln \left(\Lambda_{i} T_{0} / \rho_{c}^{1 / 2}\right)$, parametrically the same as for the flatness problem, eq. (2.15). In fig. 5 we show by the dotted curve the more realistic evolution of the horizon when reheating is more gradual. It is clear that $\Delta N$ is reduced in this case since inflation ends earlier. A careful derivation [14] shows that the number of $e$-foldings until the end of inflation, at the time when a scale of comoving wave number $k_{*}$ crosses outside the horizon, is given by

$$
N_{*} \cong 67-\ln \frac{k_{*}}{a_{0} H_{0}}+\frac{1}{4} \ln \frac{V_{*}^{2}}{m_{p}^{4} \rho_{\mathrm{end}}}+\frac{1}{12} \ln \frac{T_{\mathrm{rh}}^{4}}{\rho_{\mathrm{end}}}
$$

where $V_{*}$ is the value of $V(\varphi)$ at the time of horizon crossing (see eq. (2.23) below for the definition), and $\rho_{\text {end }}$ is the value of $V$ when inflation ends. Even this formula is simplified to the case where the equation of state during reheating is that of radiation, $w=1 / 3$. The more general result can be found in $[14,13]$.

Soon after Guth's introduction of the inflationary universe, it was realized that the quantum fluctuations of $\varphi$ during inflation can account for the density perturbations necessary for growth of structure in the universe $[15,16,17,18] .{ }^{2}$ We will later show that the quantum fluctuation of the Fourier mode of the inflaton during inflation is

$$
\delta \varphi_{k}=\int d^{3} x e^{i k \cdot x} \delta \varphi(x) \sim \frac{H}{2 \pi}
$$

for any $k$, implying that the fluctuations are nearly scale invariant, which is phenomenologically important for avoiding too large fluctuations on small scales (that would produce too many black holes) or on long ones (leading to inhomogeneity at large scales).

\footnotetext{
${ }^{2}$ Of these references, I find the last one to be the most understandable and still worth reading; it forms the basis for the presentation given in the textbook [2]. There is incidentally an interesting story about it; it originally appeared as a preprint by Steinhardt and Turner, who were finding that inflation could not produce sufficiently large fluctuations to explain the observed structure. The corrected version came out with J.M. Bardeen, an expert on cosmological perturbations, as a coauthor. He recognized that the curvature invariant that is conserved while perturbations are outside the horizon is given by $\zeta \sim \delta \rho /(\rho+p)$ during inflation, rather than $\delta \rho / \rho$ as had been assumed in the preprint version. Since $\rho+p$ is very close to zero during slow-roll inflation, this provides a huge enhancement, needed to get the observed level of density perturbations. Some copies of the original preprint still exist.
} 


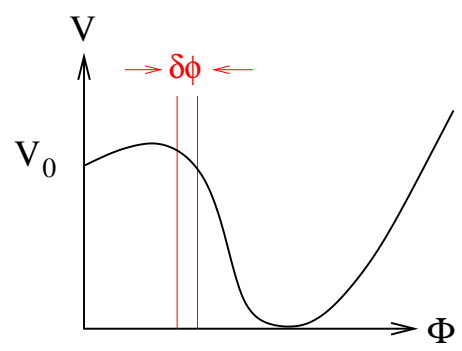

Figure 6: Quantum fluctuations of the inflaton that cause reheating to occur at different times for different places in the universe.

The inflaton fluctuations lead to density fluctuations, that can be quickly understood at a heuristic level, referring to fig. 6. Consider two regions of the universe whose value of $\varphi$ differs by $\delta \varphi$. Inflation will end slightly later in one region, with the time difference

$$
\delta t \sim \frac{\delta \varphi}{\dot{\varphi}}
$$

The differing amounts of inflation cause local perturbations in the 3D curvature of a surface at fixed time, that we can estimate as

$$
\mathscr{R}_{k} \sim \delta\left(\frac{1}{a^{2}}\right) \sim \frac{\delta a}{a} \sim H \delta t \sim \frac{H^{2}}{\dot{\varphi}}
$$

again with an approximately scale-invariant spectrum since both $H$ and $\dot{\varphi}$ change very slowly during inflation. We can relate this quantity to the potential $V$ using the slow-roll equation of motion,

$$
\frac{H^{2}}{\dot{\varphi}}=\frac{H^{3}}{H \dot{\varphi}} \sim \frac{V^{3 / 2}(\varphi)}{V^{\prime}(\varphi)}
$$

evaluated at the moment when the scale $k$ exits the horizon,

$$
\frac{k}{a} \cong k e^{-H t}=H=k e^{-N}
$$

This is the important horizon crossing condition, showing the origin of the relation $N \sim \ln k / H$ that we observe in eq. (2.18). As always, $k$ is the comoving wave number that does not change with the expansion, and whose value refers to the present time, while $k / a$ is the physical, time-dependent wave number.

An important observable quantity is the correlation function of the 3D curvature, giving rise to the scalar power spectrum $P_{s}$,

$$
P_{s}=\int d^{3} x e^{i k \cdot x}\langle\mathscr{R}(0) \mathscr{R}(x)\rangle=\left|\mathscr{R}_{k}\right|^{2} \sim \frac{H^{4}}{\dot{\varphi}^{2}} \equiv A_{s}\left(\frac{k}{k_{*}}\right)^{n_{s}-1}
$$

For historical reasons, $n_{s}=1$ is the definition of scale-invariance, known as the Harrison-Zeldovich spectrum, and from (2.24) we see that

$$
n_{s}-1=\frac{d \ln P_{s}}{d \ln k} \cong \frac{d \ln P_{s}}{d N}
$$


where we used $N=\ln k / H$ from the horizon-crossing condition (2.23) and approximated $H$ as being constant during inflation. The deviation of $n_{s}$ from 1 is important since it impacts the correlations of CMB temperature fluctuations

$$
\left\langle\frac{\delta T}{T}(0) \frac{\delta T}{T}(x)\right\rangle
$$

which leads to constraints on models of inflation.
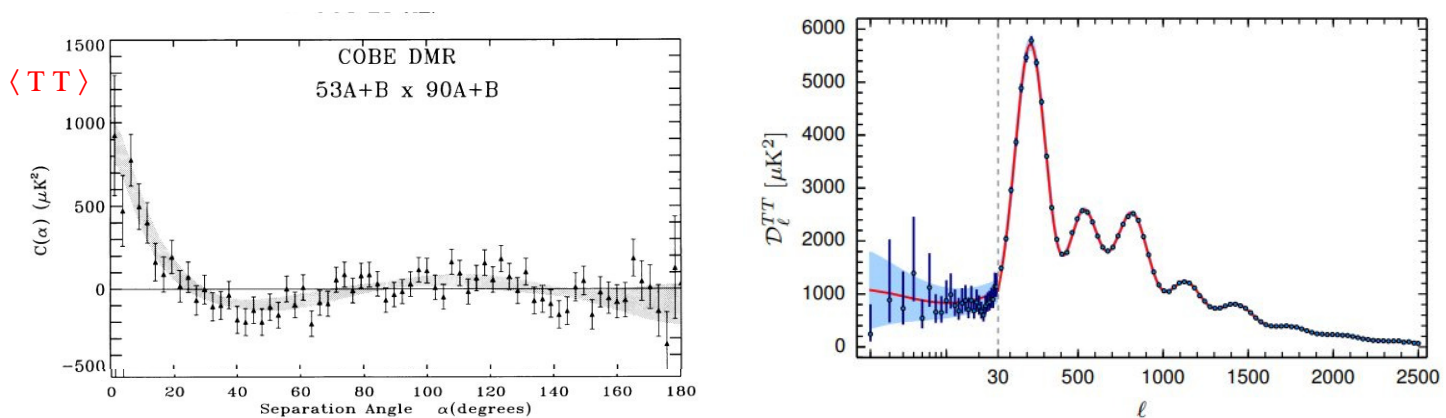

Figure 7: Left: angular correlations of the CMB temperature fluctuations, as measured by COBE [19]. Right: $\mathscr{D}_{\ell}=\ell(\ell+1) C_{\ell} / 2 \pi$ versus $\ell$ as measured by Planck [13].

In 1992 the NASA experiment COBE first observed the CMB temperature fluctuations at the level of $\delta T / T=5 \times 10^{-5}$ [19], close to the value that was already understood to be needed for consistency with structure formation. COBE measured angular correlations obtaining an oscillatory pattern as reproduced in fig. 7(left). These oscillations are better visualized in $\ell$-space by expanding in spherical harmonics,

$$
\delta T=\sum_{\ell, m} a_{\ell m} Y_{\ell m}(\theta, \varphi)
$$

and plotting

$$
C_{\ell}=\frac{1}{2 \ell+1} \sum_{m}\left|a_{\ell m}\right|^{2}
$$

versus $\ell$. This reveals the famous acoustic peaks, shown in fig. 7(right). ${ }^{3}$ They represent sound waves in the coupled photon-baryon plasma, at the time of recombination. During the tightlycoupled epoch, density perturbations $(\delta \rho / \rho)_{k}$ at a scale $k$ undergo acoustic oscillations because of the plasma pressure. But these oscillations do not begin until that scale has crossed back inside the particle horizon, which happens at different times for different scales, leading to the sound waves at different scales being out of phase with each other at the "moment" of recombination, when they start to become visible in the CMB. This process is illustrated in fig. 8.

The temperature fluctuations arise from the CMB photons climbing out of gravitational potential wells created by the density perturbations. If the perturbations were static, the net gravitational redshift would vanish, but they are evolving while the photons traverse them, leading to a net change, known as the Sachs-Wolfe effect. The detailed shape of the peaks is hard to approximate analytically, and requires solving Boltzmann equations that take into account the evolution of

\footnotetext{
${ }^{3}$ One might wonder why the COBE correlation rises at small angles while that of Planck becomes small at large $\ell$. The angular resolution of COBE was much lower than that of Planck, probing only $\ell \lesssim 25$ [20].
} 


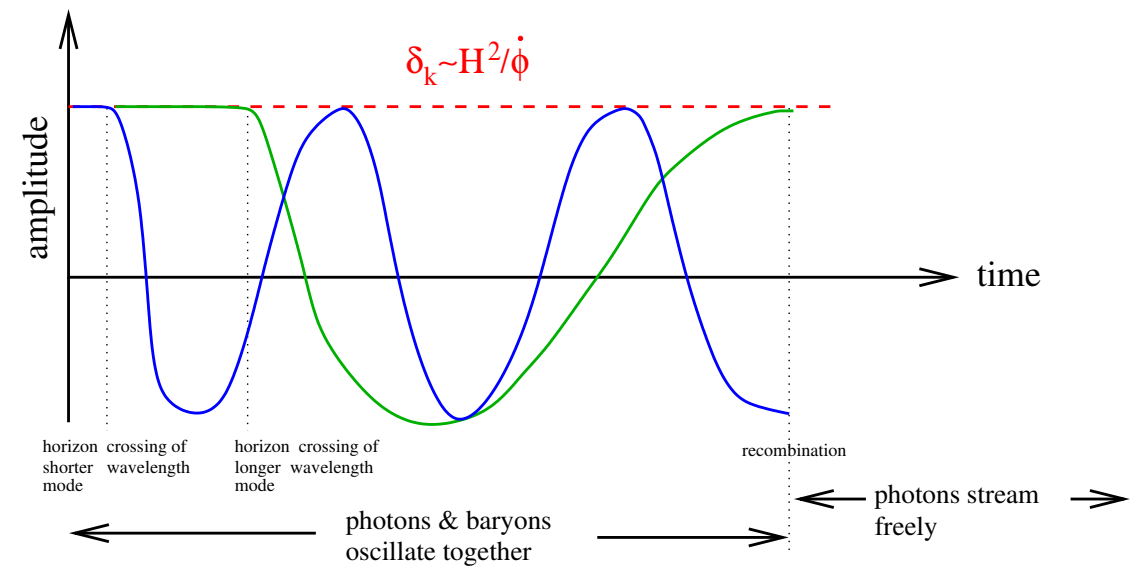

Figure 8: Illustration of the origin of CMB Doppler peaks. Sound waves of different wavelengths start oscillating with nearly the same amplitude, but at different times, when they cross back inside the horizon, $k / a=H$. Thus they are out of phase with each other at $t_{\text {rec }}$, when the universe becomes transparent and the photons start freely streaming.

the density perturbations. This is all done in publicly available codes such as CAMB, part of the CosmoMC package [21]. Clearly, the shape will be affected by the spectrum of the scalar power since it determines the slope of the line bounding the oscillations, shown as horizontal in fig. 8 . Comparison with Planck data determines the spectral index as [13]

$$
n_{s}=0.968 \pm 0.006
$$

Let's now consider the prediction for $n_{s}$ from slow roll inflation. From eq. (2.25) we find

$$
n_{s}-1=\frac{d}{d N}\left(3 \ln V-2 \ln V^{\prime}\right)=\left(3 \frac{V^{\prime}}{V}-2 \frac{V^{\prime \prime}}{V}\right) \frac{d \varphi}{d N} .
$$

Since $d N=H d t$, it follows that $d \varphi / d N=\dot{\varphi} / H$. Then using the slow roll equation (2.8) we get

$$
\frac{d \varphi}{d N}=-\frac{V^{\prime}}{3 H^{2}}=-m_{p}^{2} \frac{V^{\prime}}{V}
$$

and

$$
n_{s}-1=-6 \varepsilon+2 \eta+O\left(\varepsilon^{2}, \eta^{2}, \varepsilon \eta, \ldots\right)
$$

with higher-order slow roll parameters included in the dots.

The amplitude of the scalar power is constrained by the magnitude of the $C_{\ell}$ 's:

$$
A_{s}=\frac{H^{4}}{(2 \pi \dot{\varphi})^{2}}=\frac{V}{24 \pi^{2} m_{p}^{4} \varepsilon}=e^{3.1} \times 10^{-10}
$$

where the manner of expressing the experimental value is Planck's convention [13]. Implicit in this equation is the choice of a reference scale

$$
k_{*}=\frac{1}{20 \mathrm{Mpc}}
$$


at which all quantities are evaluated (using the horizon crossing condition to associate $k_{*}$ with a field value $\varphi_{*}$ ). One can infer from (2.33) the constraint

$$
\left|\frac{V^{3 / 2}}{m_{p}^{3} V^{\prime}}\right|=5.1 \times 10^{-4}
$$

(again at $k_{*}$ ), which fixes the overall magnitude of $V$ in a given model of inflation.

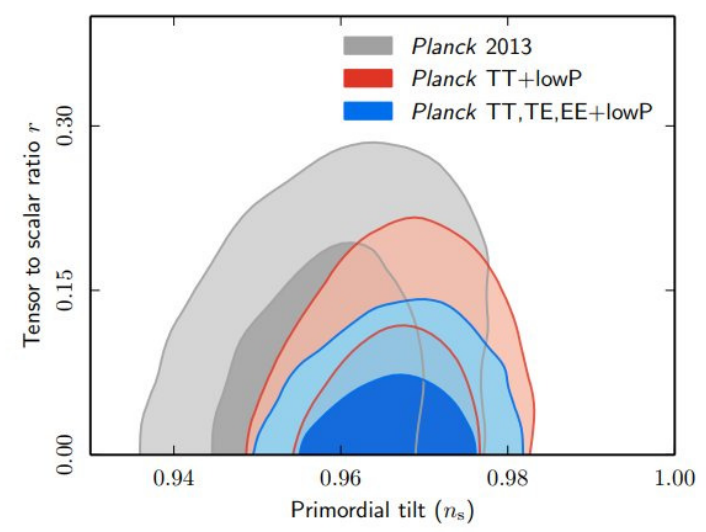

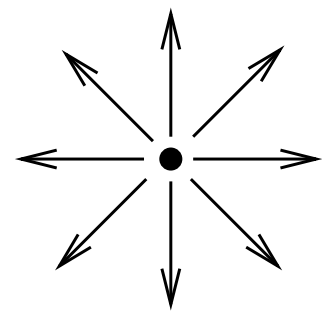

E-mode

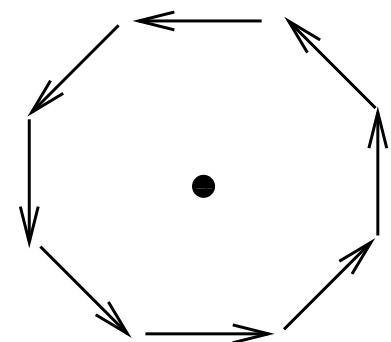

$\mathrm{B}-$ mode

Figure 9: Left: constraints on $r$ versus $n_{s}$ from ref. [13]. Right: $E$ - and $B$-mode polarizations.

In addition to the scalar perturbation, gravity waves get quantum fluctuations during inflation with the same amplitude as the inflaton field,

$$
\delta\left(h_{\mu v}\right)_{k} \sim \frac{H}{2 \pi}
$$

but with no further modulation by factors like $H / \dot{\varphi}$ since there is no potential for the graviton. The gravitational potential wells produced by these fluctuations give a separate contribution to the Sachs-Wolfe effect, beyond those coming from the density perturbations. However, they are only effective at large scales, $\ell \ll 100$ (corresponding to $k^{-1} \gg 100 \mathrm{Mpc}$ ), because at smaller scales, these tensor fluctuations re-enter the horizon before $t_{\text {rec }}$ and get Hubble damped, just like any other kind of radiation. The tensor modes that are not Hubble-damped can boost the CMB power in the blue-shaded region of fig. 7(right). The power spectrum associated with them is denoted by

$$
P_{t}=\frac{2}{m_{p}^{2}}\left(\frac{H}{2 \pi}\right)^{2} \equiv A_{t}\left(\frac{k}{k_{*}}\right)^{n_{t}-1}
$$

The tensor-to-scalar ratio is

$$
r=\frac{A_{t}}{A_{s}}=16 \varepsilon
$$

which roughly quantifies the relative contributions from tensors and scalars to the $C_{\ell}$ 's at low $\ell$. The lack of evidence for any such contribution results in the upper bound [13]

$$
r<0.1
$$


where the reference scale is smaller, $k=(500 \mathrm{Mpc})^{-1}$, appropriate to the region of $\ell$-space where the tensor contribution can be appreciable. The limit on $r$ can be translated into an upper bound on the energy scale of inflation, since eq. (2.37) depends only on $H^{2}=V / 3 m_{p}^{2}$ and not $V^{\prime}$ :

$$
V_{*}^{1 / 4} \lesssim 2 \times 10^{16} \mathrm{GeV}
$$

The combined constraints on $n_{s}$ and $r$ are shown in fig. 9(left) taken from ref. [13]. These results illustrate the importance of the separate measurements of the CMB polarization that Planck has carried out. The polarization is conventionally separated into two independent components, the $E$-mode which is curl-free and the $B$-mode which is divergenceless, illustrated in fig. 9(right). $E$-modes are produced by scalar $\delta \rho / \rho$ fluctuations, while $B$-modes are only produced by tensor perturbations (or foregrounds dominated by thermally emitting dust in the galaxy). Planck has observed $E$-modes through their cross-correlations with temperature fluctuations, $\langle\delta T E\rangle$, and their autocorrelations, $\langle E E\rangle$, and inclusion of these data strengthen the constraints. The $\langle B B\rangle$ correlations remain a holy grail of CMB detection, since they would provide more direct evidence of primordial inflationary tensor fluctuations than $r$. There was excitement when BICEP2 claimed such a detection [22], but this turned out to be dust [23].

\subsection{Example: chaotic inflation}

We now work through a specific example to show what is needed to test a model against the data, namely chaotic inflation [24]. (The name originates from a picture wherein the universe starts in a disordered state, far from the minimum of the potential, with energy density near the Planck scale. Inflation can get started in any region of size of several Planck lengths if it fluctuates into a somewhat homogeneous state. Once inflation starts, inhomogeneities are quickly damped, and the inflating regions becomes much larger than those where inflation has not yet begun.) For simplicity, the potential is taken to be monomial,

$$
V(\varphi)=\lambda m_{p}^{4}\left(\varphi / m_{p}\right)^{p}
$$

with $p>0$. The Hubble parameter is then

$$
H=\sqrt{\frac{\lambda}{3}} m_{p}\left(\frac{\varphi}{m_{p}}\right)^{p / 2}
$$

and the slow-roll equation is

$$
3 H \dot{\varphi}=3 H^{2} \frac{d \varphi}{d N}=-V^{\prime}=-\lambda p m_{p}^{3}\left(\varphi / m_{p}\right)^{p-1}
$$

We can define $N$ to be the number of $e$-foldings until the end of inflation, assuming $\varphi=\varphi_{e}$ at this time. Then eq. (2.43) can be integrated,

$$
N=-\int_{\varphi}^{\phi_{e}} d \varphi \frac{3 H^{2}}{V^{\prime}}=\frac{1}{2 p m_{p}^{2}}\left(\varphi^{2}-\varphi_{e}^{2}\right)
$$

Since $V^{\prime} / V=p / \varphi$ and $V^{\prime \prime} / V=p(p-1) / \varphi^{2}$, the slow-roll parameters are

$$
\varepsilon=\frac{p^{2}}{2}\left(\frac{m_{p}}{\varphi}\right)^{2}, \quad \eta=p(p-1)\left(\frac{m_{p}}{\varphi}\right)^{2}
$$


We see that superPlanckian field values are necessary to justify slow roll. The spectral index is then

$$
n_{s}-1=-p(p+2)\left(\frac{m_{p}}{\varphi}\right)^{2}
$$

which is always negative, called a red-tilted spectrum.

For most interesting values of $p$, inflation will end when $\varepsilon=1$ (occurring before $\eta=1$ ), giving

$$
\varphi_{e} \cong \frac{p}{\sqrt{2}} m_{p}
$$

We can then use (2.44) to reexpress $n_{s}$ as

$$
n_{s}-1=-\frac{p+2}{(2 N+p / 2)} \cong-\frac{(1+p / 2)}{N}
$$

The approximation is valid since we will be evaluating (2.48) at horizon crossing of the reference scale $k_{*}$, when $N \gg 1$.

To compare to Planck data, we must evaluate $n_{s}-1$ using the value $N_{*}$ corresponding to horizon crossing of the relevant mode $k_{*}$, eq. (2.18). The second term on the right-hand-side is $\ln k_{*} / a_{0} H_{0}=5.4$, but the third term depends upon $\lambda$, which we have not yet determined, and the last one depends upon the reheat temperature $T_{\mathrm{rh}}$, which is not known until we specify the theory more completely to determine how reheating takes place. To find $\lambda$ we need to use the normalization of the scalar power amplitude (sometimes called the COBE normalization), eq. (2.35),

$$
\frac{V^{3 / 2}}{m_{p}^{3} V^{\prime}}=\frac{\sqrt{\lambda}}{p}\left(\frac{\varphi_{*}}{m_{p}}\right)^{1+p / 2}=5 \times 10^{-4}
$$

which fixes

$$
\lambda \cong \frac{25 \times 10^{-8} p^{2}}{\left(p\left(2 N_{*}+p / 2\right)\right)^{1+p / 2}}
$$

We see that the equation for $N_{*}$ has become transcendental, but with only a weak log dependence of $N_{*}$ on the r.h.s. which can be solved by iteration. Knowing $\lambda$, we have information about the energy scale of inflation,

$$
V_{*}=\left(0.022 m_{p}\right)^{4} \frac{p}{2 N_{*}}
$$

which we see is generically not far below the Planck scale, and in danger of conflicting with the tensor bound (2.40) unless $p$ is small. This can also be seen in terms of $r$,

$$
r=16 \varepsilon=\frac{4 p}{N_{*}}
$$

which is $0.07 p$ at $N_{*}=55$, for example.

We still don't know what to take for $T_{r h}$, so the Planck collaboration and many practitioners take it to be a free parameter, letting $N_{*}$ vary between 50 and 60 to allow for the uncertainty. Given the nominal dependence $N_{*} \sim(1 / 3) \ln T_{\mathrm{rh}}$ this might seem excessively generous and you are free to make more restrictive assumptions about $T_{\mathrm{rh}}$. In any case, the resulting predictions for chaotic inflation are shown as the diagonal line segments bounded by heavy dots in fig. 10. Of the cases shown, only $p=2 / 3$ even lies in the $2 \sigma$ allowed region. Such fractional powers may seem peculiar 


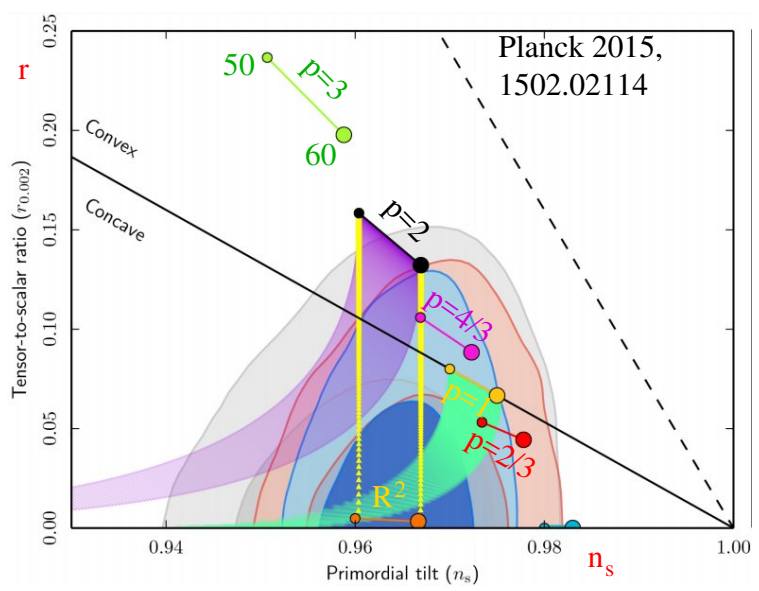

Figure 10: Predictions of chaotic inflation for $r$ versus $n_{s}$, overlaid on the Planck allowed regions.

from the point of view of renormalizable field theories, but can arise as an effective description for large values of $\varphi$ in the context of string-motivated axion monodromy models [25], for example.

The best-fitting model indicated on fig. 10 is Starobinsky's $R^{2}$ inflation [12], that can be mapped onto a scalar field inflation model with potential of the form

$$
V(\varphi)=\Lambda^{4}\left(1-\exp \left(-\sqrt{2 / 3} \varphi / m_{p}\right)\right)^{2}
$$

(we will explain how, below; see eq. (2.89)). More generally, current Planck data prefer models with a convex potential. These include Hilltop models [26]

$$
V=\Lambda^{4}\left(1-(\varphi / \mu)^{p}+\ldots\right)
$$

and Higgs inflation, where the total action is

$$
S=\int d^{4} x \sqrt{-g}\left(\mathscr{L}_{\text {grav }}+\mathscr{L}_{\mathrm{SM}}+\xi R|H|^{2}\right)
$$

As we will show at the end of this chapter, this can be transformed to scalar potential similar to that of $R^{2}$ gravity by going to the Einstein frame.

Sometimes one prefers to solve the coupled inflaton/Friedmann equations numerically instead of using the slow roll approximation (see [27]) for details), particularly if the inflaton has a noncanonical kinetic term $\frac{1}{2} f(\varphi) \dot{\varphi}^{2}$, or if there are several fields. This can be done efficiently in terms of the canonical field momentum

$$
\pi=\frac{\delta \mathscr{L}}{\delta \dot{\varphi}}=f(\varphi) \dot{\varphi}
$$

Then

$$
H=\frac{1}{\sqrt{3} m_{p}}\left(\mathscr{L}_{\text {kin }}+V\right)^{1 / 2}
$$

and the equations of motion can be written in first-order form

$$
\begin{aligned}
& \frac{d \pi}{d N} \equiv \pi^{\prime}=-3 \pi+\frac{1}{H} \frac{\partial}{\partial \varphi}\left(\mathscr{L}_{\text {kin }}-V\right) \\
& \frac{d \varphi}{d N} \equiv \varphi^{\prime}=\frac{\pi}{f H}
\end{aligned}
$$




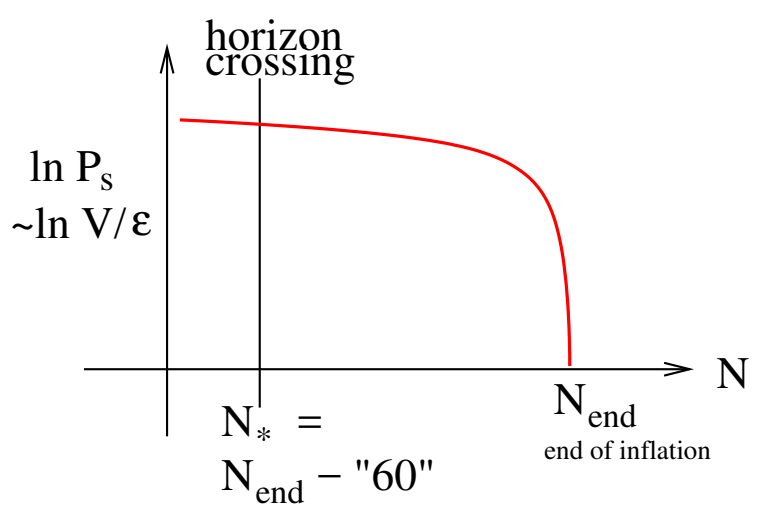

Figure 11: Typical dependence of the scalar power on the number of $e$-foldings in a numerical solution.

The end of inflation is easy to recognize since the fields start oscillating around the minimum of the potential; this behavior can typically be followed for numerous periods of oscillations before the Runge-Kutta step size shrinks to zero and creates an exception in the code. The exact expression for the spectral index is

$$
n_{s}-1=\frac{d \ln P_{s}}{d \ln k}=\frac{d \ln P_{s}}{d N}\left(1+\frac{d \ln H}{d N}\right)^{-1}
$$

where the correction term $(\ldots)^{-1}$ is usually very close to unity. The quantity $\ln P_{s}$ as a function of $N$ will resemble fig. 11. By evaluating it at $N_{*}$ (using eq. (2.18)) one can match to the observed spectrum.

\subsection{Filling in some details}

The previous section was meant to give an intuitive understanding of inflation, and to allow you to start confronting your favorite model against data. Here we would like to sketch some of the details that would be needed for a deeper or more rigorous understanding. Some useful references for this material include $[28,29,30,31]$ and others we will cite below.

\subsubsection{Quantum fluctuations of the inflaton}

First we want to explain the origin of the quantum fluctuation $\delta \varphi=H / 2 \pi$. This is straightforward to derive, by canonically quantizing a free scalar field in an FRW background, that we can approximate as de Sitter space. The expansion of $\varphi$ in Fourier modes looks the same as in flat space,

$$
\varphi(x)=\int \frac{d^{3} k}{(2 \pi)^{3 / 2}}\left(a_{k} \psi_{k}(x)+a_{k}^{\dagger} \psi_{k}^{*}(x)\right)
$$

except that the mode functions now have a different time dependence,

$$
\psi_{k}=e^{i \vec{k} \cdot \vec{x}} f_{k}(t)
$$

where (using $a=e^{H t}$ for a pure dS background),

$$
\ddot{f}_{k}+3 H \dot{f}_{k}+\left(m^{2}+k^{2} e^{-2 H t}\right) f_{k}=0
$$


If $\varphi$ is the inflaton then necessarily $m^{2} \ll H^{2}$ during inflation, to have slow roll, and $k / a=k e^{-H t}>$ $H$ before horizon crossing. Hence we can ignore the mass and solve (2.62) in the $m=0$ limit. Defining $z=k e^{-H t} / H$, the solution can be written

$$
\begin{aligned}
f_{k} & =C z^{3 / 2}\left(J_{3 / 2}(z)+i Y_{3 / 2}(z)\right) \\
& =-C \sqrt{\frac{2}{\pi}} z(i+z) e^{i z}
\end{aligned}
$$

with a normalization constant $C$ to be determined. The particular combination of the independent solutions $J_{3 / 2}$ and $Y_{3 / 2}$ was chosen with hindsight, since we want this to agree with the usual Minkowski space solution in the limit $H \rightarrow 0$, where up to an irrelevant phase

$$
f_{k} \rightarrow C \sqrt{\frac{2}{\pi}} \frac{k}{H} e^{-i k t}
$$

This shows that we took the right linear combination, and it fixes the normalization constant to be

$$
C=\frac{H}{k} \sqrt{\frac{\pi}{4 k}}
$$

Now we can compute the r.m.s. fluctuations of $\varphi$ using the usual property of the creation and annihilation operators, $\left\langle 0\left|a_{k} a_{k^{\prime}}^{\dagger}\right| 0\right\rangle=\delta^{(3)}\left(\vec{k}-\vec{k}^{\prime}\right)$ :

$$
\left\langle 0\left|\varphi^{2}(x)\right| 0\right\rangle=\int \frac{d^{3} k}{(2 \pi)^{3}}\left|f_{k}\right|^{2}=\int \frac{d^{3} k}{(2 \pi)^{3}} \frac{H^{2}}{2 k^{3}}\left(1+\frac{k^{2}}{H^{2}} e^{-2 H t}\right)
$$

The second term is just the usual UV-divergent contribution already present in Minkowski space, as can be seen from the fact that the factors of $H$ cancel out, and $e^{-2 H t}$ can be absorbed by rescaling $k \rightarrow k_{\text {phys }} e^{H t}$. We don't care about this term because it can be removed by renormalization, and in any case its contributions are only important at distance scales that are much too small to be cosmologically relevant. This first term however is new, and is associated with being in the dS background. The structure $d^{3} k / k^{3}$ shows that equal power is present in the fluctuations from every logarithmic interval of $k$, and this corresponds to a scale-invariant spectrum.
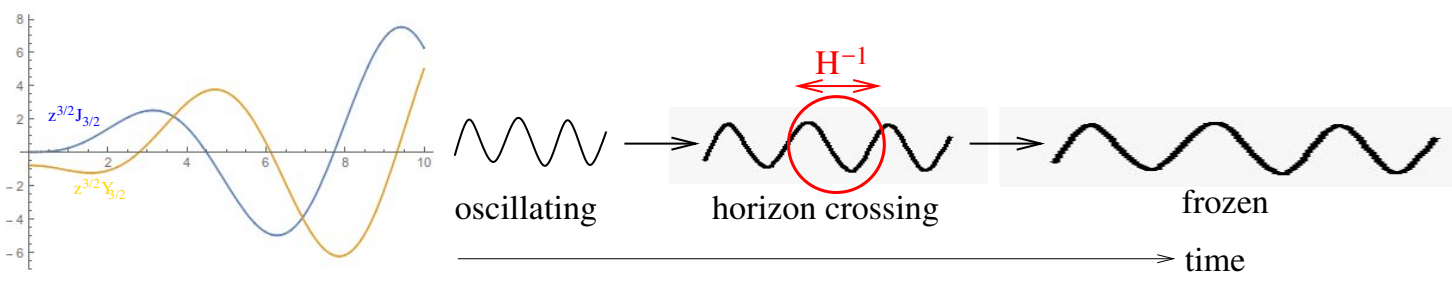

Figure 12: Left: time-dependence of the inflation fluctuation modes, as a function of $z=k e^{-H t} / H$. Right: illustration of the freezing of the mode functions following horizon crossing.

The shape of the solutions (2.63) can give some insight into the significance of horizon crossing. Fig. 12(left) shows that the mode functions stop oscillating at lates times (small z), starting around $z=1$, which is when the corresponding wavelength goes outside the horizon. Thereafter 
they remain "frozen" until later reentry into the horizon. This behavior is illustrated more qualitatively in fig. 12(right). It is argued [32] that horizon crossing corresponds to the transition between quantum and classical behavior of the fluctuations. We can express the classical field as

$$
\varphi(x)=\int \frac{d^{3} k}{(2 \pi)^{3}} \varphi_{k} e^{i k \cdot x}
$$

having a volume-averaged fluctuation

$$
\left\langle\varphi^{2}\right\rangle=\frac{1}{V} \int d^{3} x \varphi^{2}(x)=\frac{1}{V} \int \frac{d^{3} k}{(2 \pi)^{3}}\left|\varphi_{k}\right|^{2}
$$

Comparison with (2.66) shows that therefore $\varphi_{k}=H \sqrt{V / 2 k^{3}}$, which leads to a scale-invariant power spectrum,

$$
P_{\varphi} \propto k^{3}\left|\varphi_{k}\right|^{2} \quad \Longleftrightarrow \quad\left\langle\varphi^{2}\right\rangle \sim \int \frac{d k}{k} P_{\varphi}
$$

The equal power in each logarithmic interval of scales implies that every $e$-folding of inflation also contributes equal power.

\subsubsection{Cosmological perturbation theory}

Our description of fluctuations is still over-simplified, since $\delta \varphi$ induces perturbations in the metric that cannot be ignored. To properly understand the interplay requires cosmological perturbation theory, which is reviewed in the references by Liddle and Lyth, as well as [33, 34, 35]. The general perturbation to the metric can be written as

$$
\delta g_{\mu \nu}=\left(\begin{array}{c|c}
2 \phi & -2 a^{2} B_{, i} \\
\hline-2 a^{2} B_{, i} & 2\left(\psi \delta_{i j}+E_{, i j}\right) a^{2}
\end{array}\right)
$$

These perturbations can be decomposed in Lorentz scalars, vectors, and tensors (gravity waves). Vector perturbations always decay in expanding space and we therefore neglect them.

By gauge transformations (diffeomorphisms),

$$
x^{\mu} \rightarrow x^{\mu}+\xi^{\mu}(x)
$$

some of the functions in (2.70) can be set to zero. For example in the conformal Newtonian, a.k.a. longitudinal gauge,

$$
E,=B_{, i}=0
$$

and furthermore the perturbed Einstein equations imply $\psi=\phi$ as long as there is no anisotropic stress, $T_{i j}=T_{j i}$. Another popular choice of gauge is the comoving one, in which the inflaton fluctuation is defined to vanish (by demanding the surfaces of constant time have uniform energy density),

$$
\delta \phi=0, \quad B_{, i}=0
$$

An important quantity is the curvature perturbation $\mathscr{R}$, defined by generalizing the Friedmann equation to include the effects of inhomogeneity:

$$
H^{2}(x, t)=\frac{\rho(x, t)}{3 m_{p}^{2}}+\frac{2}{3} \frac{\nabla^{2}}{a^{2}} \mathscr{R}
$$


It is related to the $3 \mathrm{D}$ curvature of the spatial surfaces on comoving foliations as

$$
R^{(3)}=\frac{4 k^{2}}{a^{2}} \mathscr{R}
$$

in Fourier space, and it coincides with Bardeen's $\zeta$ variable mentioned previously. The utility of $\mathscr{R}$ is that it does not evolve for superhorizon modes in single-field inflation (where entropy and anisotropic stress perturbations vanish). Therefore if one can compute it at horizon exit, then the same value applies at the moment of horizon reentry, at which time the equations of classical linear perturbation evolution take over (to predict large scale structure or temperature fluctuations).

The expression for $\mathscr{R}$ in terms of metric perturbations generally depends upon the choice of gauge, but it can be written in way that is independent of the gauge [36]

$$
\mathscr{R}=-\left(\Psi+\frac{H}{\dot{\phi}} \Delta \varphi\right)
$$

where

$$
\begin{aligned}
\Psi & \equiv \phi+\frac{1}{a}\left[\left(B-E^{\prime}\right) a\right]^{\prime} \\
\Delta \varphi & =\delta \varphi+\left(B-E^{\prime}\right) \varphi^{\prime}
\end{aligned}
$$

and prime denotes the derivative with respect to conformal time $\eta$, with $d t=a d \eta$. To rigorously compute the quantum fluctuations of the inflaton, one must quantize $\mathscr{R}$ since the canonically normalized field is a linear combination of metric and inflaton perturbations (see also [37]). However the magnitude of this fluctuation turns out to be the same as in the simplified approach, giving a scalar power spectrum that is nearly scale invariant with amplitude going as $H^{4} / \dot{\phi}^{2}$.

\subsection{Variations on the simplest inflation models}

Thus far we have assumed inflation is driven by a single scalar field with nothing too exotic about its Lagrangian, and perturbative decays of the inflaton after inflation. To finish this lecture on inflation I would like to mention some slightly more complicated scenarios that have been widely studied.

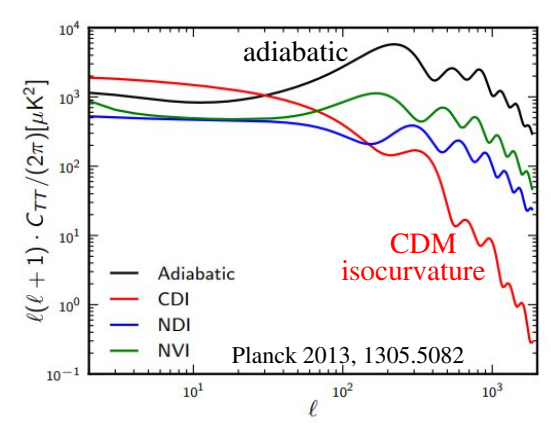

Figure 13: Comparison of CMB Doppler peaks predicted by pure isocurvature versus adiabatic perturbations. Adapted from ref. [38]. 


\subsubsection{Isocurvature fluctuations}

The density perturbations we have considered so far are called "adiabatic," such the perturbations in separate components (baryons, cold dark matter, photons, neutrinos) are related by

$$
\frac{\delta \rho_{b}}{\rho_{b}}=\frac{\delta \rho_{\mathrm{CDM}}}{\rho_{\mathrm{CKM}}}=\frac{3}{4} \frac{\delta \rho_{\gamma}}{\rho_{\gamma}}=\frac{3}{4} \frac{\delta \rho_{v}}{\rho_{v}}
$$

The factor of $3 / 4$ can be understood from the fact that there is a single temperature fluctuation controlling the densities:

$$
\begin{gathered}
\rho_{\gamma} \sim T^{4}, \quad \Longrightarrow \frac{\delta \rho_{\gamma}}{\rho_{\gamma}}=4 \frac{\delta T}{T}, \\
\rho_{\mathrm{CDM}} \sim m T^{3}, \quad \Longrightarrow \frac{\delta \rho_{\mathrm{CDM}}}{\rho_{\mathrm{CDM}}}=3 \frac{\delta T}{T}
\end{gathered}
$$

and this is sourced by the curvature fluctuation $\mathscr{R}$.

In multifield inflation models, it is possible to have fluctuations of different particle species that violate the adiabatic condition. An orthogonal possibility is that particle number fluctuates between different species in a way that keeps the total $\delta \rho$ and $\mathscr{R}$ equal to zero. In such a case, the entropy is perturbed, hence these are known as entropy or isocurvature fluctuations.

In multifield inflation, at least two particles are approximately massless, $m_{i}^{2} \ll H^{2}$, but isocurvature perturbations can arise even if only one field controls inflation, while a second light field eventually contributes significantly to the energy density of the universe. An example is the axion. Its isocurvature fluctuation can be quantified in terms of its number density, compared to the total entropy density $s$, through the parameter

$$
S_{a}=\frac{\delta\left(n_{a} / s\right)}{n_{a} / s}=\frac{\delta n_{a}}{n_{a}}-3 \frac{\delta T}{T} \cong \frac{\delta n_{a}}{n_{a}}
$$

which would vanish for an adiabatic perturbation. The neglect of $\delta T / T$ follows from the fact that $\delta \rho \sim m_{a} \delta n_{a}+\delta T n_{\gamma}=0$ for an entropy fluctuation [2], and $\rho_{a} \ll \rho$. A very small temperature fluctuation can compensate for $\delta n_{a}$ in the total energy density to keep $\delta \rho=0$, since only one degree of freedom is being compensated by many.

Running the CMB Boltzmann codes with isocurvature rather than adiabatic fluctuations gives a pattern of acoustic peaks that look very different, in strong disagreement with observations, as illustrated in fig. 13. Analysis shows that isocurvature modes can contribute no more than about $7 \%$ of the total perturbation. Such considerations rule out low values of the axion decay constant, depending upon the assumed Hubble rate during inflation [13].

An interesting variation can occur if the isocurvature fluctuation is able to decay later on and convert to adiabatic form, for example by decay into radiation. This opens the possibility that the inflaton fluctuation could much smaller than normally assumed, and the perturbations all arise from a second field, known as the curvaton [39].

\subsubsection{Nongaussianity}

The adiabatic fluctuations in standard inflation behave as a Gaussian random variable, to a good approximation, with higher-point correlation functions being slow-roll suppressed:

$$
\left\langle(\delta \phi)^{3}\right\rangle \sim V^{\prime \prime \prime}
$$


The nonlinearity of gravity also induces nongaussian correlations, but these are Planck-suppressed. The corresponding CMB temperature correlations $\left\langle(\delta T)^{3}\right\rangle$ are thus expected to be very small. Any nongaussian correlations should ultimately arise from their counterparts in the curvature perturbation, the bispectrum

$$
\left\langle\zeta_{k_{1}} \zeta_{k_{2}} \zeta_{k_{3}}\right\rangle=f\left(\vec{k}_{1}, \vec{k}_{2}\right)
$$

which depends upon two independent wave vectors since translational invariance implies $\sum \vec{k}_{i}=0$.

In multifield models of inflation, or single field models with complicated kinetic terms, significant nongaussianity can arise. A simple way of parametrizing it is to define a nongaussian curvature perturbation contructed from the Gaussian one via a nonlinearity parameter $f_{\mathrm{NL}}$ [40]

$$
\zeta_{\mathrm{NG}}=\zeta+f_{\mathrm{NL}}\left(\zeta^{2}-\left\langle\zeta^{2}\right\rangle\right)
$$

so that

$$
\langle\zeta \zeta \zeta\rangle \sim f_{\mathrm{NL}}\langle\zeta \zeta\rangle^{2}
$$

This gives a phenomenological way of characterizing the level of nongaussianity that typical models like multi-field inflation might predict, although the $k_{i}$-dependence ("shape") of the bispectrum may not match the simple form predicted by (2.84) in a given model. Planck currently constraints $\left|f_{\mathrm{NL}}\right| \lesssim 10$, depending upon the shape. Theorists continue to explore the possible implications of a future detection of nongaussianity; see for example [41].

\subsubsection{Preheating}

Perturbative decay of the inflaton is a relatively inefficient reheating mechanism, in the sense that typically the reheat temperature is suppressed, $T_{\mathrm{rh}} \ll \Lambda_{i}$. More efficient means of particle production can occur in the background of the oscillating inflation, namely parametric resonance, which can occur much faster than perturbative decay [42, 43, 44, 45]. This mechanism can work even if the inflaton is a stable particle, allowing for the possibility that the inflaton could be the dark matter [46]. For certain kinds of inflaton potentials (e.g., $\varphi^{n}$ with $n>4$ during the reheating stage), conventional reheating may be particularly inefficient, and superseded by gravitational particle production, a mechanism that only relies upon the change in the time-dependence of the scale factor between inflation and conventional FRW expansion [47].

\subsection{Current outlook}

The inflationary models that currently give the best fit to data seem to be ones having a nonminimal coupling of the inflaton to gravity:

$$
\mathscr{L}_{J}=\sqrt{-g_{J}}\left(\frac{1}{2} R_{J}\left(m_{p}^{2}+\xi \varphi^{2}\right)+\frac{1}{2}(\partial \varphi)^{2}-V(\varphi)\right)
$$

where the subscript $J$ denotes that we are in the Jordan frame, in which the gravitational constant varies with time due to the evolution of $\varphi$. Cosmology is best understood by going to the Einstein frame via a Weyl rescaling of the metric,

$$
g_{E}^{\mu \nu}=\frac{g_{J}^{\mu \nu}}{\Omega^{2}}, \quad \Omega^{2}=1+\xi \varphi^{2} / m_{p}^{2}
$$


This induces a complicated kinetic term for $\varphi$, and the canonically normalized inflaton $\chi$ is related to $\varphi$ by

$$
\frac{d \chi}{d \varphi}=\left(\frac{\Omega^{2}+6 \xi^{2} \varphi^{2} / m_{p}^{2}}{\Omega^{4}}\right)^{1 / 2}
$$

In the Einstein frame, the Lagrangian reads

$$
\mathscr{L}_{E}=\sqrt{-g_{E}}\left(\frac{1}{2} m_{p}^{2} R_{E}+\frac{1}{2}(\partial \chi)^{2}-\frac{V}{\Omega^{4}}\right)
$$

The nice thing about this is that it makes any renormalizable potential $V$ convex, favored by Planck data. In particular if $V \sim \varphi^{4}$ at large field values, $V / \Omega^{4}$ becomes flat. This feature enables allows one to identify the standard model Higgs as the inflaton [48], or to rescue models of chaotic inflation with large values of the exponent [49].

Even the best fitting model, Starobinsky's $R^{2}$ inflation, can be understood in this way, by introducing a dimensionless auxiliary scalar field to the Einstein-Hilbert action, with the Lagrangian [50]

$$
\mathscr{L}=\frac{1}{2} m_{p}^{2}(1+\varphi) R-\frac{3}{4} M^{2} m_{p}^{2}(\varphi-1)
$$

Integrating out $\varphi$ trivially leads to the $R^{2}$ term in the gravitational action,

$$
\mathscr{L} \rightarrow \frac{1}{2} m_{p}^{2}\left(R+\frac{R^{2}}{6 M^{2}}\right)
$$

But instead of integrating it out, one can transform to the Einstein frame, which generates a kinetic term for $\varphi$ and gives the canonically normalized inflaton a potential of the form

$$
V(\chi) \sim m_{p}^{2} M^{2}\left(1-e^{-\sqrt{2 / 3} \chi / m_{p}}\right)^{2}
$$

with the predicted spectral index and tensor ratio

$$
n_{s}-1 \cong-\frac{2}{N_{*}}, \quad r \cong \frac{12}{N_{*}^{2}} \approx 10^{-4}
$$

\subsection{Exercises}

\section{Scalar field in FRW background.}

(a) Write the Lagrangian for a scalar field in a FRW gravitational background and show that its equation of motion is

$$
\ddot{\varphi}+3 H \dot{\varphi}-\frac{1}{a^{2}} \nabla^{2} \varphi=-\frac{\partial V}{\partial \varphi}
$$

where $H=\dot{a} / a$.

(b) Now consider a free massive scalar, with frequency $\omega \gg H$. Using the ansatz

$$
\varphi=\varphi_{0} \exp \left(-i\left[\int^{t} d t \omega-\vec{k} \cdot \vec{x}\right]-f(t)\right)+\text { c.c. }
$$


show that $\omega= \pm \sqrt{(k / a)^{2}+m^{2}}$ gives a solution, with $f \sim \ln a$ if $m \ll H \ll k / a$ and $f \sim(3 / 2) \ln a$ if $m \gg H$.

(c) The stress-energy tensor is

$$
T_{\mu v}=\frac{\delta S}{\delta g^{\mu \nu}}=\partial_{\mu} \varphi \partial_{v} \varphi-\eta_{\mu v} \mathscr{L}
$$

Using the solution from (b), find $\left\langle T_{\mu v}\right\rangle$, averaging over oscillations. Average over directions of $\vec{k}$ to verify that $p=\rho / 3$ if $m \ll k / a$ and $p \cong 0$ if $m \gg k / a$. Also show that $\rho \sim 1 / a^{4}$ or $1 / a^{3}$ respectively, for these two cases.

(d) A gas of relativistic scalar particles has $\rho=\pi^{2} T^{4} / 30$. If the classical solution (b) gets thermalized into particles, how is $T$ related to the parameters of that solution? Repeat for a nonrelativistic gas with $\rho \approx(m T / 2 \pi)^{3 / 2}$.

2. Perturbative reheating. At the end of inflation, the Hubble parameter is

$$
H=\frac{1}{\sqrt{3} m_{p}}\left(\rho_{\varphi}+\rho_{r}\right)^{1 / 2}
$$

where the oscillating and decaying inflaton has energy density

$$
\rho_{\varphi}=\frac{V_{e}}{a^{3}} e^{-\Gamma t}
$$

if $V_{e}$ was the scalar field energy density at the end of inflation and $\Gamma$ is the decay rate. (For convenience we take $a=1$ and $t \cong 0$ at the end of inflation.) $\rho_{r}$ is the energy density of the radiation that is produced by the decays. It satisfies the Boltzmann equation

$$
\frac{d}{d t}\left(a^{4} \rho_{r}\right)=a^{4} \Gamma \rho_{\varphi}
$$

where the factor $a^{4}$ accounts for the redshifting and dilution of the radiation in a comoving volume $a^{3}$. At times before $1 / \Gamma$, it is a good approximation to ignore $\rho_{r}$ compared to $\rho_{\varphi}$ in the Friedmann equation, because of the redshifting, and also to approximate $e^{-\Gamma t / 2} \cong 1$. Use these approximations to simultaneously solve the two equations and show that $\rho_{r} \sim(T / a)^{4}$ at late times $\gtrsim 1 / \Gamma$, with the dependence on $V_{e}$ cancelling out.

3. Hilltop inflation. Analyze the hilltop inflation model, $V=\Lambda^{4}\left(1-(\varphi / \mu)^{2}\right)$ in the slow-roll approximation, assuming that horizon crossing of the relevant mode occurs at $N_{*}=60$. Hint: show that $\eta$ dominates the spectral index, but $\varepsilon$ controls the end of inflation. Find $\mu$ and $\Lambda$ from the best fit to $n_{s}$ and $A_{s}$, as well as $\varphi_{*}$ (the value of $\varphi$ at horizon crossing) and the predicted tensor-to-scalar ratio $r$.

4. Slow roll as attractor. The scalar field equation of motion is second order and has two independent solutions, while the slow-roll approximation is first order and has only one. Find the two independent solutions for the case where the initial condition is far from the slow-roll solution, and show that they quickly decay, leaving only the slow-roll solution. Hint: expand the EOM around $\varphi=\varphi_{s r}+\delta \varphi$ to linear order in $\delta \varphi$ and show that it satisfies

$$
\ddot{\delta} \varphi+(3+\varepsilon) H \dot{\delta} \varphi-3(\varepsilon-\eta) H^{2} \delta \varphi=\frac{1}{3}(\varepsilon-\eta) V^{\prime}
$$


where $H$ and $V^{\prime}$ are evaluated at $\varphi_{s r}$. Then solve it using problem 1, treating $H$ as approximately constant, and neglecting the small inhomogeneous term.

5. A step potential. Suppose $V$ has a sudden step when $\varphi$ crosses $\varphi_{0}$,

$$
V=\left\{\begin{array}{l}
a \varphi+V_{2}, \varphi>\varphi_{0} \\
b \varphi+V_{1}, \varphi \leq \varphi_{0}
\end{array}\right.
$$

(a) From the scalar equation of motion, derive an equation for the discontinuity in $\dot{\varphi}$ when $\varphi$ crosses $\varphi_{0}$.

(b) Solve the slow-roll EOM on both sides of the step, and note that $\dot{\varphi}$ does not have the right discontinuity. However a linear combination of the transient solutions you found in problem 4 can fix this problem; find it. Note that it should be applied only after crossing the step, since one can presume the field was slowly rolling before it reached the step.

(c) The step leads to a modification of the power of the curvature or density fluctuations at the scale corresponding to horizon crossing when $\varphi$ passes the step. What does it look like?

\section{Baryogenesis}

According to our timeline, fig. 2, the next likely important event of early cosmology may have been leptogenesis, or baryogenesis, depending upon when the dark matter was produced. In principle, the reheat temperature could have been too low for leptogenesis. BBN tells us that $T_{\mathrm{rh}} \gtrsim 1 \mathrm{MeV}$ at the lowest, but it is hard to imagine creating the baryon asymmetry at such low temperatures. In the following highly abbreviated account, I will focus on leptogenesis and electroweak baryogenesis as two popular theories for the origin of matter.

The present universe is observed to contain essentially only matter and no antimatter, except for the rare antiparticles produced by cosmic rays. There is an asymmetry between baryons and antibaryons (called the baryon asymmetry of the universe, BAU) that can be expressed as

$$
\eta=\frac{n_{B}-n_{\bar{B}}}{n_{\gamma}}=\left\{\begin{array}{r}
{[5.8-6.6] \times 10^{-10}, \text { from BBN }} \\
(6.09 \pm 0.06) \times 10^{-10}, \text { from CMB }
\end{array}\right.
$$

or

$$
\frac{n_{B}-n_{\bar{B}}}{s}=\frac{\eta}{7.04}
$$

using the entropy density for reference. In the context of inflation it would be hard to imagine this asymmetry arising as an initial condition, since entropy was generated during reheating, but any preexisting baryon asymmetry was only diluted by the expansion of the universe, by a factor of $e^{-3 N}$. It is therefore assumed that the BAU was created after inflation.

\subsection{History}

Sakharov is acknowledged as the first to seriously consider baryogenesis, in 1967 [51], and three necessary conditions for creating the BAU are attributed to him: $B$ violation, departure from thermal equilibrium, and violation of $C$ and $C P$. In the paper, he did not spell out these conditions as being necessary, but rather put forward a rather vague model of superheavy particle decay leading 
to a baryon asymmetry, mentioning that the decays would violate thermal equilibrium, and noting the need for $B$ and $C P$ violation. There is no clear statement about the necessity of all these ingredients. It was only around 1978-79 that their status started to be clarified. Ref. [52] presented a model of $B$ - and $C P$-violating out-of-equilibrium decays of a second Higgs doublet, in which the three ingredients are clearly stated in the first paragraph, and Sakharov is cited. (In 1979 Sakharov himself wrote a paper that was similar in spirit, in that it enunciated the conditions at the outset, and cited the 1967 work, but in the context of GUTs.)

Outside of the Soviet Union, it took slightly longer to appreciate the need for going out of thermal equilibrium. Yoshimura [53], probably unaware of Sakharov's work, proposed that decay of GUT bosons could produce the asymmetry, but he overlooked the out-of-equilibrium criterion, and only by neglecting a cancelling contribution obtained a nonzero result. Its necessity was rigorously proven in ref. [54] (also unaware of Sakharov) in a proposal for baryogenesis via black hole evaporation. That reference also clearly shows the need for $C, C P$ and $B$ violation.

We will proceed by commenting on the three requirements.

\subsection{The three laws}

\subsection{1 $B$ violation}

Although baryon number is a symmetry of the SM Lagrangian, it is violated at the quantum level through the triangle anomaly. The baryon number current is

$$
J_{B}^{\mu}=\frac{1}{3} \sum_{f} \bar{q}_{f} \gamma^{\mu} q_{f}
$$

where the sum is over quark flavors, and its divergence is [55]

$$
\partial_{\mu} J_{B}^{\mu}=-\frac{g_{2}^{2}}{64 \pi^{2}} \varepsilon^{\mu \nu \rho \sigma} W_{\mu \nu}^{a} W_{\rho \sigma}^{a}
$$

in the presence of a background $\mathrm{SU}(2)_{L}$ gauge field with strength $W_{\mu \nu}^{a}$.

At zero temperature, the $B$-violating effects are through nonperturbative instanton configurations involving the Higgs field and the $W$ fields, whose action is $S \sim 8 \pi^{2} / g_{2}^{2} \sim 187$. Hence the tunneling rate per unit volume is of order

$$
\frac{\Gamma}{V} \sim v^{4} e^{-2 S} \sim 10^{-160} v^{4}
$$

where $v \sim 100 \mathrm{GeV}$ represents the weak scale. Hence the number of baryon decays in the observable universe, over its lifetime, would be

$$
H_{0}^{-4} \frac{\Gamma}{V} \sim 10^{-25}
$$

if only zero-temperature transitions were relevant. But at high temperatures $T \gtrsim v$, electroweak symmetry is restored and there is no barrier between the $N$-vacua of the $\mathrm{SU}(2)_{L}$ gauge theory. Transitions between these vacua result in the creation of quarks and leptons such that $B$ and lepton number $L$ both change by 3 units, one per generation [56]. For details, see for example [57].

At high temperatures, sphalerons are the nonperturbative static field configurations that violate $B$ and $L$. One can think of the sphaleron as the top of the energy barrier separating the $N$-vacua, 
at temperatures below the electroweak phase transition (EWPT). Above the transition, when the barrier disappears, the sphaleron is no longer a well-defined field configuration, but the same kind of $B$ - and $L$-violating transitions still occur, mediated by field configurations that have the same topological properties as the low-temperature sphaleron. These transitions are no longer exponentially suppressed since there is no tunneling; they are only suppressed by powers of the weak gauge coupling. One can visualize the sphaleron transitions by a Feynman diagram with 9 left-handed quarks and 3 left-handed leptons in the external states, even though there is no analytic expression for the corresponding amplitude, at high $T$. Instead, lattice studies have determined the rate per volume of these transitions [58],

$$
\frac{\Gamma}{V}=(1.05 \pm 0.08) \times 10^{-6} T^{4}
$$

In a thermal volume $V=1 / T^{3}$ (which contains on average one particle of each type in the early universe plasma), the corresponding rate goes as $T$, while the Hubble rate goes as $H \sim T^{2} / m_{p}$. This implies that sphalerons come into equilibrium as $T$ falls below $10^{13} \mathrm{GeV}$, and only go back out of equilibrium at the EWPT when the exponential suppression comes into effect.

Therefore one of the criteria for baryogenesis is already present in the SM, provided that $T_{\mathrm{rh}} \gtrsim 100 \mathrm{GeV}$ following inflation. We will focus on two paradigms for baryogenesis that take advantage of this, but let's mention a few other possible sources of $B$ violation from physics beyond the SM. It seems likely that such interactions should exist, since $B$ is only an accidental symmetry of the SM: the particle content and gauge symmetries of the SM forbid any $B$-violating operator of dimension $\leq 4$.

In the SU(5) GUT there are gauge bosons $X$ and $X^{\prime}$ with interactions of the form

$$
\begin{aligned}
& X_{\mu} \bar{l}_{l} \gamma^{\mu} d_{R}^{c}, \quad X_{\mu} \bar{u}_{R}^{c} \gamma^{\mu} q_{L}, \quad X_{\mu} \bar{q}_{L} \gamma^{\mu} e_{R}^{c} \\
& X_{\mu}^{\prime} \bar{L}_{l} \gamma^{\mu} u_{R}^{c}, \quad X_{\mu}^{\prime} \bar{d}_{R}^{c} \gamma^{\mu} q_{L}
\end{aligned}
$$

where the superscript $c$ denotes charge conjugation. Clearly there is no consistent way to assign baryon number to $X_{\mu}$ or $X_{\mu}^{\prime}$. This source of $B$ violation provided an early example of baryogenesis through out of equilibrium decays of the heavy bosons [59].

In supersymmetry (SUSY) it is possible to write $B$-violating operators if $R$-parity is relaxed. A superpotential term of the form

$$
\lambda^{\prime \prime} U D D
$$

gives rise to potential terms in which two of the right-handed superfields are replaced by the corresponding quark, while the third becomes a squark. If $L$-violating operators are for some reason forbidden, or sufficiently small, then proton decay will be suppressed despite the presence of the $U D D$ interactions.

\subsection{Loss of thermal equilibrium}

Departure from thermal equilibrium is needed to get a BAU, since any process in equilibrium has the same rate as its time-reversed process, by definition. The out of equilibrium decay of a heavy particle like the $X$ or $X^{\prime}$ of SU(5) GUTs is a good example. Suppose that $X$ can decay in such a way as to produce more $B$ than $\bar{B}$. The inverse decays will simply undo this if the decays are 
in equilibrium. To quantify it, we must consider the Boltzmann equation for $n_{B}$, which we define to be the difference in densities of baryons and antibaryons. For simplicity, focus on the $X^{\prime}$ decays, and assume that there are equal numbers of $X^{\prime}$ and its antiparticle $\bar{X}^{\prime}$ decaying in the plasma. There must be a $C P$ asymmetry in the decays, such that the net baryon number per decay is given by

$$
\varepsilon=\frac{\frac{1}{3} \Gamma\left(X^{\prime} \rightarrow l u\right)-\frac{2}{3} \Gamma\left(X^{\prime} \rightarrow \bar{q} \bar{d}\right)-\frac{1}{3} \Gamma\left(\bar{X}^{\prime} \rightarrow \bar{l} \bar{u}\right)+\frac{2}{3} \Gamma\left(\bar{X}^{\prime} \rightarrow q d\right)}{\Gamma\left(X^{\prime} \rightarrow \text { any }\right)+\Gamma\left(\bar{X}^{\prime} \rightarrow \text { any }\right)}
$$

The Boltzmann equation is

$$
\begin{aligned}
\frac{1}{a^{3}} \frac{d}{d t}\left(a^{3} n_{B}\right) & =\dot{n}_{B}+3 H n_{B} \\
& =\left(n_{X^{\prime}}+n_{\bar{X}^{\prime}}\right) \Gamma_{X^{\prime}} \varepsilon-\sum_{\substack{y, z \\
X^{\prime} \text { or } \bar{X}^{\prime}}} B_{y+z} n_{y} n_{z} \sigma_{\substack{y+z \rightarrow \\
X^{\prime} \circ \mathrm{r} \bar{X}^{\prime}}} v_{\mathrm{rel}}
\end{aligned}
$$

where $y, z$ denote the different possible initial states of the inverse decays. The bottom line contains the collision terms of the Boltzmann equation, and they cancel each other, by definition, when the decays (and inverse decays) are in thermal equilibrium, giving $\dot{n}_{B}=0$.

The form of (3.11) makes it mysterious that two such different looking terms could exactly cancel each other. The original form of the collision term in terms of amplitudes $\mathscr{M}$ makes it clear however. Consider the contribution from a single channel, say $X^{\prime} \rightarrow l u$. We label $X^{\prime}$ as particle $1, l$ and $u$ as 2 and 3. Defining the Lorentz-invariant phase space element as $d \Pi_{i}=d^{3} p /\left[(2 \pi)^{3}(2 E)\right]$, the contribution to the collision term is

$$
\delta \mathscr{C}=\prod_{i} \int d \Pi_{i}\left[f_{1}\left(1-f_{2}\right)\left(1-f_{3}\right)-f_{2} f_{3}\left(1+f_{1}\right)\right]|\mathscr{M}|^{2}(2 \pi)^{4} \delta^{(4)}\left(p_{1}-p_{2}-p_{3}\right)
$$

where $f_{i}$ are the Bose-Einstein or Fermi-Dirac distribution functions, $\left(e^{\beta E} \pm 1\right)^{-1}$, and we have included the effect of Pauli blocking or Bose enhancement in the final states. The term in square brackets can be written as

$$
[\ldots]=f_{1} f_{2} f_{3}\left[e^{\beta\left(E_{2}+E_{3}\right)}-e^{\beta E_{1}}\right]
$$

using $1-f=e^{\beta E} f$ for fermions and $1+f=e^{\beta E} f$ for bosons. Clearly (3.13) vanishes by energy conservation, imposed by the delta function. But of course we have assumed that the distribution functions are those corresponding to thermal equilibrium to get this result.

If $X^{\prime}$ decays out of equilibrium, its true distribution function is not Bose-Einstein, which would imply its number density is

$$
n=\frac{g}{(2 \pi)^{3}} \int \frac{d^{3} p}{e^{\beta E}-1} \cong g\left(\frac{m T}{2 \pi}\right)^{3 / 2} e^{-m / T}
$$

for $T \ll m$. ( $g=3$ is the number of degrees of freedom for a massive vector.) Instead it is much larger,

$$
n \sim \frac{g \zeta(3)}{\pi^{2}} T^{3} e^{-\Gamma t}
$$

that is, the same as a radiation degree of freedom, except for the particles that disappeared due to decays. Therefore we can, in some approximation, ignore the contribution of the inverse decays to the collision term to estimate the baryon asymmetry. 
Instead of using $a^{3} n$ as the dependent variable (which is not a bad choice since it remains constant under the Hubble expansion, in the absence of collisions), it is convenient to use the proportional quantity called the abundance

$$
Y_{i}=\frac{n_{i}}{s}
$$

where $s=(\rho+p) / T$ is the entropy density,

$$
s=\frac{2 \pi^{2}}{45} g_{*, s} T^{3}
$$

with

$$
g_{*, s}=\sum_{i} g_{i}\left(\frac{T_{i}}{T}\right)^{3} \times\left\{\begin{array}{l}
1, \text { bosons } \\
\frac{7}{8}, \text { fermions }
\end{array}\right.
$$

which allows for different species to have temperatures $T_{i}$ different from that of the photon (due to kinetic decoupling of that species).

If we ignore the inverse decay term then the Boltzmann equation for the baryon abundance simplifies to

$$
\dot{Y}_{B} \cong 2 Y_{X^{\prime}} e^{-\Gamma t} \Gamma \varepsilon
$$

which can readily be solved to yield the final abundance

$$
\left.Y_{B}\right|_{t \rightarrow \infty}=\left.2 \varepsilon Y_{X^{\prime}}\right|_{\text {initial }}
$$

This is exactly as we would expect from the fact that each $X^{\prime}$ or $\bar{X}^{\prime}$ decay leads on average to $\varepsilon$ baryons.

To make a better estimate, we can keep the inverse decay term in the equation, and quantify it using the detailed balance argument,

$$
\dot{Y}_{B}=2 \varepsilon \Gamma\left(Y_{X^{\prime}}(t)-Y_{X^{\prime}}^{\mathrm{eq}}(t)\right)
$$

which implies the collision term must vanish when $X^{\prime}$ is in equilibrium. Its actual abundance can be estimated as $Y_{X^{\prime}, i} e^{-\Gamma t}$ as before (where the initial value is $Y_{X^{\prime}, i} \cong 3 / g_{*, s} \sim 10^{-2}$, since $X^{\prime}$ is approximately three degrees of freedom out of $g_{*, s}$ total, $\sim 320$ for supersymmetric SU(5) GUT), while the equilibrium abundance is approximately

$$
Y_{X^{\prime}}^{\mathrm{eq}}(t) \cong Y_{X^{\prime}, i} \begin{cases}1, & T>m \\ \left(\frac{m}{T}\right)^{3 / 2} e^{-m / T}, & T<m\end{cases}
$$

It is convenient to define a new time variable, $x=m / T$; then

$$
t=\frac{0.301 M_{p}}{\sqrt{g_{*}} T^{2}}=\frac{0.301 M_{p}}{\sqrt{g_{*}} m^{2}} x^{2}=\frac{x^{2}}{2 H(m)}
$$

where $M_{p}=1.22 \times 10^{19} \mathrm{GeV}$ is the unreduced Planck mass, and $m$ is the mass of $X^{\prime}$. The last equality follows from the fact that $H=1 / 2 t$ during radiation domination, where $H(m)$ denotes the Hubble parameter when $T=m$. One can integrate (3.21) to find that

$$
Y_{B}(\infty)=\frac{\varepsilon Y_{X^{\prime}} \Gamma}{H(m)}\left[2 \frac{H(m)}{\Gamma}-O(1)\right]
$$

We leave this as an exercise. It shows that the washout correction is small as long as $\Gamma \ll H(m)$. This is exactly the condition for the decays to be out of equilibrium: the reaction rate must fall below the expansion rate. 


\subsection{1 $C, C P$ violation}

In the previous example the role of the asymmetry parameter $\varepsilon$ was clear. It is the quantity that vanishes if charge conjugation $C$ or its combination with parity, $C P$, is conserved. Let's recall how fields transform under these discrete symmetries. A Dirac fermion $\psi$ goes as

$$
\begin{aligned}
P: & \psi=\left(\begin{array}{c}
\psi_{L} \\
\psi_{R}
\end{array}\right) \rightarrow\left(\begin{array}{l}
\psi_{R} \\
\psi_{L}
\end{array}\right)(t,-\vec{x}) \\
C: & \psi \rightarrow\left(\begin{array}{c}
\sigma_{2} \psi_{L}^{*} \\
-\sigma_{2} \psi_{R}^{*}
\end{array}\right)(t, \vec{x}) \\
C P: & \psi \rightarrow\left(\begin{array}{c}
\sigma_{2} \psi_{R}^{*} \\
-\sigma_{2} \psi_{L}^{*}
\end{array}\right)(t,-\vec{x})
\end{aligned}
$$

while for a complex vector $X^{\mu}$,

$$
\begin{gathered}
P: \quad X^{\mu} \rightarrow\left(X^{0},-X^{i}\right)(t,-\vec{x}) \\
C: \quad X^{\mu} \rightarrow-\left(X^{\mu}\right)^{*}(t, \vec{x}) \\
C P: \quad X^{\mu} \rightarrow\left(-X^{0}, X^{i}\right)^{*}(t,-\vec{x})
\end{gathered}
$$

One can show that under $C P$, a Lagrangian allowing for $X \rightarrow \bar{\chi} \psi$ decays transforms as

$$
\lambda \bar{\psi} X^{\mu} \gamma_{\mu} \chi+\lambda^{*} \bar{\chi} \bar{X}^{\mu} \gamma_{\mu} \psi \rightarrow \lambda^{*} \bar{\psi} X^{\mu} \gamma_{\mu} \chi+\lambda \bar{\chi} \bar{X}^{\mu} \gamma_{\mu} \psi
$$

Therefore one needs a complex coupling, $\lambda \neq \lambda^{*}$, to have $C P$ violation. This is exactly how $C P$ violation comes into the standard model Lagrangian, via the CKM matrix in the $W$ boson couplings to quark currents.

While complex couplings are a necessary condition for $C P$ violation, they are not sufficient. Notice that at tree level, the decay rates $\Gamma(X \rightarrow \psi \bar{\chi})$ and $\Gamma(\bar{X} \rightarrow \bar{\psi} \chi)$ both go like $|\lambda|^{2}$, where the phase is irrelevant. In fact the phase is unphysical in the simple model (3.27) since it can be removed by a field redefinition,

$$
X^{\mu} \rightarrow e^{-i \theta} X^{\mu} \quad \text { or } \quad \chi \rightarrow e^{-i \theta} \chi \quad \text { or } \quad \psi \rightarrow e^{i \theta} \psi
$$

where $\lambda=|\lambda| e^{i \theta}$.
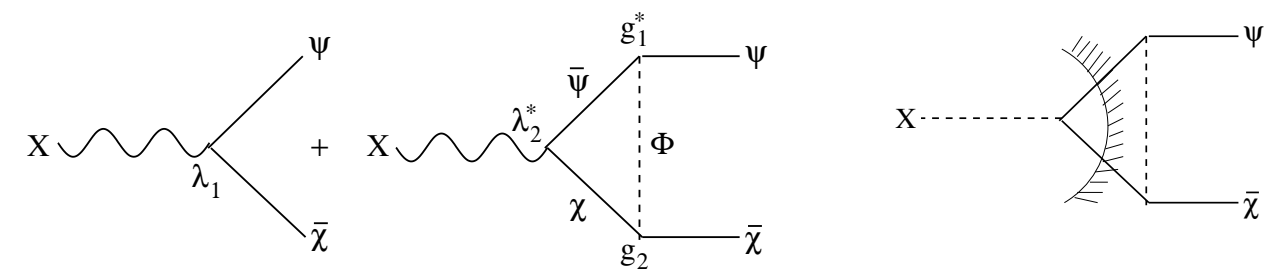

Figure 14: Left: toy model for generating $C P$ asymmetry in $X \rightarrow \psi \bar{\chi}$ versus $\bar{X} \rightarrow \bar{\psi} \chi$ decays. Right: cut version of loop, for applying the Cutkosky rule.

To make a phase physical, we need more interactions-enough so that not all phases can be removed by field redefinitions. For example

$$
\lambda_{1} \bar{\psi} X \chi+\lambda_{2} \bar{\psi} \bar{X} \chi+g_{1} \Phi \bar{\psi} \psi^{c}+g_{2} \Phi \bar{\chi} \chi^{c}+\text { h.c. }
$$


where $\Phi$ could be a real or complex scalar. Now consider the decay $X \rightarrow \psi \bar{\chi}$ including one-loop corrections, as shown in fig. 14(left). The interference between tree and loop diagrams produces the $C P$ asymmetry,

$$
\varepsilon=\frac{\left|\mathscr{M}_{X \rightarrow \psi \bar{\chi}}\right|^{2}-\left|\mathscr{M}_{\bar{X} \rightarrow \bar{\psi} \chi}\right|^{2}}{\left|\mathscr{M}_{X \rightarrow \psi \bar{\chi}}\right|^{2}+\left|\mathscr{M}_{\bar{X} \rightarrow \bar{\psi} \chi}\right|^{2}}
$$

because the loop diagram gets an imaginary part, predicted by the optical theorem or unitarity of the $S$-matrix. This tells us that the matrix element has an imaginary part

$$
2 \operatorname{Im} \mathscr{M}_{X \rightarrow \psi \bar{\chi}}=\sum_{\substack{\bar{\psi} ; \\ \text { spins }}} \int d \Pi_{\bar{\psi}} d \Pi_{\chi} \mathscr{M}_{X \rightarrow \bar{\psi} \chi} \mathscr{M}_{\bar{\psi} \chi \rightarrow \psi \bar{\chi}}
$$

The r.h.s. corresponds to the loop diagram shown in fig. 14(right) with the internal propagators "cut" (put on their mass shell) according to the Cutkosky rule [60], where the cut propagators are replaced by

$$
\frac{i}{p^{2}-m^{2}+i \varepsilon} \rightarrow 2 \pi \theta\left(p_{0}\right) \delta\left(p^{2}-m^{2}\right)
$$

This rule also applies to fermionic propagators once they have been rationalized, $i(\not p-m+i \varepsilon)^{-1}=$ $i(\not p+m) /\left(p^{2}-m^{2}+i \varepsilon\right)$, i.e., just multiply everything by $(\not p+m)$.

As a consquence, the form of the tree plus loop contributions for the respective decays is

$$
\begin{aligned}
& \mathscr{M}_{X \rightarrow \psi \bar{\chi}} \sim \lambda_{1}+\lambda_{2}^{*} g_{1}^{*} g_{2}(A+i B), \\
& \mathscr{M}_{\bar{X} \rightarrow \bar{\psi} \chi} \sim \lambda_{1}^{*}+\lambda_{2} g_{1} g_{2}^{*}(A+i B),
\end{aligned}
$$

The important point is that there is no complex conjugation of $A+i B$, which arises purely from the kinematics of the loop. We then infer

$$
\varepsilon \cong \frac{i B}{\left|\lambda_{1}\right|^{2}}\left(\lambda_{1} \lambda_{2} g_{1} g_{2}^{*}-\text { c.c. }\right)=-\frac{2 B}{\left|\lambda_{1}\right|^{2}} \operatorname{Im}\left(\lambda_{1} \lambda_{2} g_{1} g_{2}^{*}\right)
$$

In this example, the $C P$-violating phase that is invariant under field redefinitions is the argument of $\lambda_{1} \lambda_{2} g_{1} g_{2}^{*}$, so indeed we needed all four couplings nonvanishing to have $C P$ violation in this toy model.

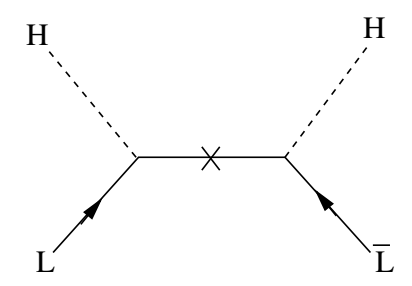

Figure 15: Neutrino mass operator induced by integrating out heavy sterile neutrinos. 


\subsection{Leptogenesis}

Leptogenesis is one of the most popular models for explaining the baryon asymmetry, because it requires hardly any new physics ingredients beyond those needed to explain neutrino masses by the seesaw mechanism. This is most simply accomplished by introducing several heavy righthanded neutrinos $N_{i}$, singlets under the SM gauge group, that couple to lepton doublets and the Higgs via

$$
y_{v, i j} \bar{L}_{i} \tilde{H} N_{R, j}+\text { h.c. }
$$

where $\tilde{H}=i \tau_{2} H^{*}=\left(H^{0 *},-H^{-}\right)^{T}$. The heavy neutrinos have Majorana masses (without loss of generality we can work in the mass eigenbasis for the $N_{i} \mathrm{~s}$ ):

$$
\frac{1}{2} M_{j} \bar{N}_{j} N_{j}^{c}
$$

Integrating out the heavy $N_{j}$ produces the dimension-5 Weinberg operator

$$
\left(y_{v} M^{-1} y_{v}^{T}\right)_{i j}\left(\bar{L}_{i} \tilde{H}\right)\left(\tilde{H}^{T} L_{j}^{c}\right)
$$

as shown in fig. 15. Electroweak symmetry breaking then gives the light neutrino mass matrix

$$
m_{v, i j}=v^{2}\left(y_{v} M^{-1} y_{v}^{T}\right)_{i j}
$$

with $v=246 \mathrm{GeV}$.

The cross in fig. 15 denotes an insertion of the heavy neutrino mass in the internal propagator, to explain the clash of arrows showing the flow of lepton number. The Majorana mass term (3.36) is the only interaction in the theory that violates lepton number (by two units), and so it must be involved. Therefore only the $M /\left(p^{2}-M^{2}\right)$ part of the propagator $(\not p+M) /\left(p^{2}-M^{2}\right)$ contributes. Of course $p^{2}$ is negligible at low energy so we can neglect it in the denominator. There are also analogous loop diagrams with no mass insertions or clashes of arrows, that correct the real part of the amplitude, but do not contribute to $C P$ violation.

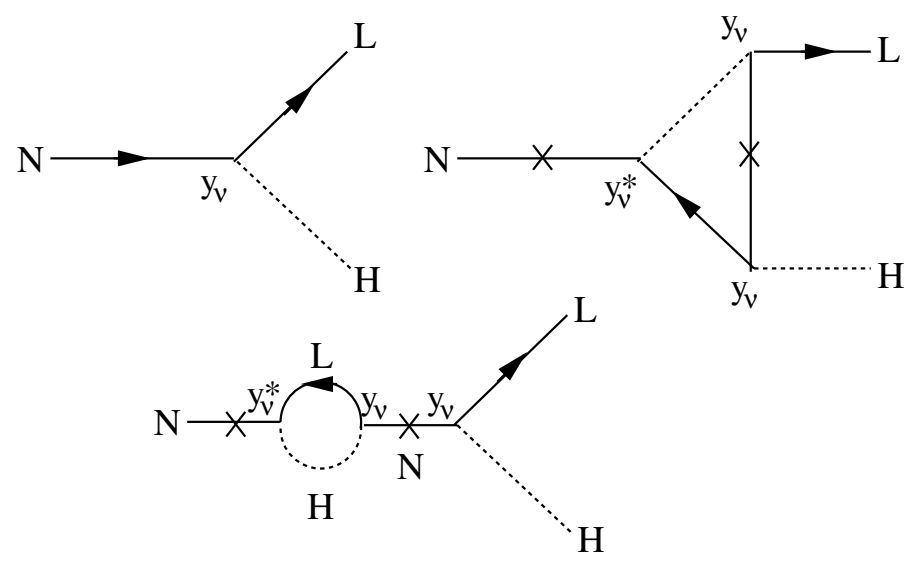

Figure 16: $C P$ asymmetric decay of heavy sterile neutrinos for leptogenesis.

The out-of-equilibrium decays of $N_{j} \rightarrow L_{i} H^{c}$ can create a lepton asymmetry by the mechanism illustrated in our toy model. The relevant diagrams are given in fig. 16. This produces a lepton 
asymmetry, which sphaleron interactions will partially convert into the baryon asymmetry, with $Y_{B} \sim-\frac{1}{3} Y_{L}$. There are many useful reviews of leptogenesis, including [61, 62]. I will give a brief synopsis of the main results here.

First, the $C P$ asymmetry for decays of $N_{j}$ can be computed from the diagrams in fig. 16 as in the toy model, by interfering the tree-level and cut loop diagrams. The result takes the form

$$
\varepsilon_{j}=-\frac{3}{8 \pi} \sum_{i \neq j} \frac{\operatorname{Im}\left[\left(y_{v}^{\dagger} y_{v}\right)_{i j}^{2}\right]}{\left(y_{v}^{\dagger} y\right)_{j j}} f\left(\frac{M_{j}}{M_{i}}\right)
$$

where $f$ is the loop function. In the case of hierarchical heavy neutrino masses, with $M_{1} \ll M_{2,3}$, $f \rightarrow M_{1} / M_{i}$. It is obvious that we need at least two families of $N_{j}$ since if $i=j=1$ there is no imaginary part. By considering all possible forms for the neutrino Dirac mass matrix, Davidson and Ibarra derived a famous bound in the hierarchical case [63],

$$
\left|\varepsilon_{1}\right| \leq \frac{3}{16 \pi} \frac{M_{1}}{v^{2}}\left(m_{3}-m_{2}\right)
$$

in terms of the light neutrino mass eigenvalues $m_{i}$. Atmospheric neutrino oscillations fix $m_{3}^{2}-m_{2}^{2} \sim$ $(0.05 \mathrm{eV})^{2}$, so if the light $v$ mass spectrum is also hierarchical, the bound (3.40) implies

$$
\left|\varepsilon_{1}\right| \lesssim \frac{M_{1}}{2 \times 10^{16} \mathrm{GeV}}
$$

If $M_{1} \ll M_{2,3}$, we expect that $N_{1}$ decays will give the dominant contribution to the lepton asymmetry, because of $\Delta L=2$ washout processes mediated by $N_{1}$ exchange, shown in fig. 15 where $N_{1}$ is the virtual state. This interaction tends to relax the asymmetry from $N_{2,3}$ decays toward zero until $T<M_{1}$, when it starts to go out of equilibrium. By this time the heavier neutrinos are long gone. But this washout process also reduces the asymmetry from the $N_{1}$ decays and must be included in the Boltzmann equations to get an accurate result. One can consider two coupled equations, one for the lepton abundance and one for that of $N_{1}$, which take the form [61]

$$
\begin{aligned}
\frac{d Y_{N_{1}}}{d x} & =(-D+S)\left(Y_{N_{1}}-Y_{N_{1}}^{\mathrm{eq}}\right) \\
\frac{d Y_{L}}{d x} & =-\varepsilon_{1} D\left(Y_{N_{1}}-Y_{N_{1}}^{\mathrm{eq}}\right)-W Y_{L}
\end{aligned}
$$

Here $D$ is the decay rate, $S$ is the rate of $\Delta L=1$ scatterings (for example $N L \rightarrow t Q_{3}$ mediated by $H$ exchange in the $s$-channel), and $W$ is the washout rate from both $\Delta L=1$ and 2 scatterings.

The out-of-equilibrium criterion for the $N_{1}$ decays can be quantified in terms of an "effective neutrino mass" $\tilde{m}_{1}$ and an "equilibrium neutrino mass" $m_{*}$,

$$
\tilde{m}_{1} \equiv \frac{v^{2}}{M_{1}}\left(y_{v}^{\dagger} y_{v}\right)_{11} \quad<\quad m_{*} \equiv \frac{16 \pi^{5 / 2}}{3 \sqrt{5}} \sqrt{g_{*}} \frac{v^{2}}{M_{p}} \cong 10^{-3} \mathrm{eV}
$$

$\tilde{m}_{1}$ is only "effective" because it has the wrong kind of indices (belonging to the heavy right-handed neutrinos) to be part of the light neutrino mass matrix. We emphasize that the inequality need not be literally satisfied; instead one will pay a penalty in the produced asymmetry going as $m_{*} / \tilde{m}_{1}$ if it is not, on which we will elaborate shortly. 
Recall our naive estimate in the toy model that

$$
Y_{L} \sim \frac{\varepsilon}{g_{*, s}}
$$

based on the idea that each $N_{1}$ decay yields on average $\varepsilon$ leptons. This ignores the effect of washout, but it can be corrected by introducing an "efficiency factor" $\kappa$,

$$
Y_{L} \sim \kappa \frac{\varepsilon}{g_{*, s}}
$$

which can be calculated in a given model by numerically solving the Boltzmann equations. One finds that $\kappa$ can be fit to an approximate formula [61]

$$
\kappa \cong 0.02\left(\frac{0.01 \mathrm{eV}}{\tilde{m}_{1}}\right)
$$

if $\tilde{m}_{1} \gtrsim 10^{-3} \mathrm{eV}$, which is known as the "strong washout regime." In this case, $N_{1}$ is decaying not far out of equilibrium, so the washout processes are very effective, and the lepton asymmetry is accordingly reduced. Why would one want to work in this inefficient regime? It has the advantage of offering the nice approximation (3.46), and moreover it is more predictive because the final lepton asymmetry is not sensitive to initial conditions, including the reheat temperature, as is the case in the weak washout regime. And besides, great efficiency is not needed because the baryon asymmetry is quite small.

Putting these results together, we find the correct magnitude of the BAU if $M_{1} \gtrsim 3 \times 10^{10} \mathrm{GeV}$ and $\left(y_{v}^{\dagger} y_{v}\right)_{11}^{1 / 2} \gtrsim 0.002$. These have the right order of magnitude to agree with the observed mass difference $m_{2}^{2}-m_{1}^{2}=(0.0086 \mathrm{eV})^{2}$. Hence leptogenesis seems to be a very natural and plausible theory of baryogenesis. Its main weakness is that it does not make any unambiguous predictions for low-energy observables. For example, there are 6 unremovable phases in $y_{v, i j}$ if there are three families of $N_{j}$, but only 3 phases in the PMNS matrix that describes light neutrino mixing.

There exist variants of standard leptogenesis that are more testable. One possibility is to use a more complicated version of the seesaw mechanism, called inverse seesaw [64] that allows a natural explanation of the light neutrino masses using a much lower scale of heavy Majorana masses. Thus the new physics needed for leptogenesis can come down to testable scales [65]. Another way is to have nearly degenerate $M_{i}$. Then the loop function $f\left(M_{1} / M_{i}\right)$ can be resonantly enhanced, to values of order $y_{v}^{-2}$, allowing $\varepsilon_{j} \sim 1$ and $M_{i}$ at the TeV scale. This is known as resonant leptogenesis [66]. I leave as an exercise to show that for quasi-degenerate $M_{i}$,

$$
\varepsilon_{i} \sim \sum_{j} \frac{\operatorname{Im}\left(y_{v}^{\dagger} y_{v}\right)_{i j}^{2}}{\left(y_{v}^{\dagger} y_{v}\right)_{i i}\left(y_{v}^{\dagger} y_{v}\right)_{j j}} \frac{\delta M_{i j}^{2} M_{i} \Gamma_{j}}{\left(\delta M_{i j}^{2}\right)^{2}+M_{i}^{2} \Gamma_{j}^{2}}
$$

where $\delta M_{i j}^{2}=M_{i}^{2}-M_{j}^{2}$ and $\Gamma_{j}$ is the decay rate of $N_{j}$. Resonant leptogenesis makes predictions for low energy lepton-violating processes like neutrinoless double beta decay, $\mu \rightarrow e \gamma, \mu \rightarrow 3 e$. The TeV-scale heavy neutrinos might be produced at a future $e^{+} e^{-}$or $\mu^{+} \mu^{-}$collider [67].

\subsection{Electroweak baryogenesis}

A more testable scenario relies on the electroweak phase transition (EWPT) being first order, to achieve the out-of-equilibrium condition. Bubbles of true vacuum, with $\langle H\rangle=v / \sqrt{2}$ in their 
interior, nucleate during such a transition, and the sphaleron rate $\Gamma_{\mathrm{sph}}$ is exponentially suppressed inside the bubbles. If $v(T) / T \gtrsim 1$ at the time of nucleation, $\Gamma_{\text {sph }}$ is small enough to avoid washout of a baryon asymmetry produced by $C P$-violating interactions at the bubble walls. In the standard model, the EWPT is a crossover transition, not first order. New physics at the weak scale is needed to make it first order.

Moreover new sources of $C P$ violation beyond the SM are needed to produce a large enough BAU. A simple argument involves the Jarlskog determinant [], which is an invariant measure of the $C P$-violating phase in the $\mathrm{SM}$, analogous to the r.h.s. of (3.34) in our toy model,

$$
J=\operatorname{det}\left[m_{u}^{2}, m_{d}^{2}\right] \sim \sin \delta \times f\left(\left|V_{C K M}\right|, m_{u_{i}}^{2}-m_{d_{j}}^{2}\right)
$$

where $m_{u}^{2}$ and $m_{d}^{2}$ are the mass matrices of the up- and down-type quarks, $\delta$ is the phase in the CKM matrix, and $f$ is a function of the magnitudes of $V_{C K M}$ matrix elements and quark mass eigenvalues. This quantity has dimension 12 , so the dimensionless measure of $C P$ violation should be $J / v^{12} \sim 10^{-20}$ since $v=246 \mathrm{GeV}$ is presumably the relevant scale. This is too small of course. A valiant attempt to lower the relevant scale was made in ref. [68, 69], but this turned out to be spoiled by quantum decoherence by finite temperature scattering [70].

\subsubsection{The $C P$ asymmetry}

For electroweak baryogenesis (EWBG), the $C P$ violation needs to lead to a $C P$ asymmetry $\delta f_{C P}=f_{L}-f_{R}$ in some species of SM fermions: for example an excess of left-handed (LH) versus right-handed top quarks, in the symmetric phase outside of the bubble where they are massless and it makes sense to talk about chirality [71, 72]. Sphalerons, which interact only with the LH particles, would like to reduce the excess in LH particles (minus their antiparticles). This necessarily converts some $O(1)$ fraction of $\delta f_{C P}$ into a baryon asymmetry. For example, suppose that $\delta f_{C P}$ is intially an excess in $t_{L}: \delta f_{t_{L}}=-\delta f_{t_{R}}=\delta f_{C P}$. Sphalerons can reduce $\delta f_{t_{L}}$ by roughly $1 / 2$ by partially converting it into $\bar{d}_{L}$ and $\bar{s}_{L}$ asymmetries, for example, that couples the sphaleron to each generation. The net result would be

$$
\delta f_{t_{L}} \rightarrow \delta f_{C P} / 2, \quad \delta f_{d_{L}}=\delta f_{s_{L}}=-\delta f_{C P} / 2
$$

This arrangement increases the entropy, and produces a baryon asymmetry since the net baryon number is now proportional to

$$
\delta f_{t_{L}}+\delta f_{d_{L}}+\delta f_{s_{L}}+\delta f_{t_{R}}=\left(\frac{1}{2}-\frac{1}{2}-\frac{1}{2}-1\right) \delta f_{C P}
$$

Charge is conserved by producing the neutrinos in this example. This argument is heuristic; we will give proper equations below.

Computing the $C P$ asymmetry $\delta f_{C P}$ is not easy in EWBG, and it is also controversial because the community is split between two formalisms. The one I prefer, because it is known to be a controlled approximation, is the semiclassical force approach [73, 74]. It usually involves a fermion whose mass varies spatially across the bubble wall (taken to be the $z$ direction), with Lagrangian

$$
\mathscr{L}=\bar{q}\left(i \not \partial-\left[m_{r}(z)+i m_{i}(z) \gamma_{5}\right]\right) q
$$




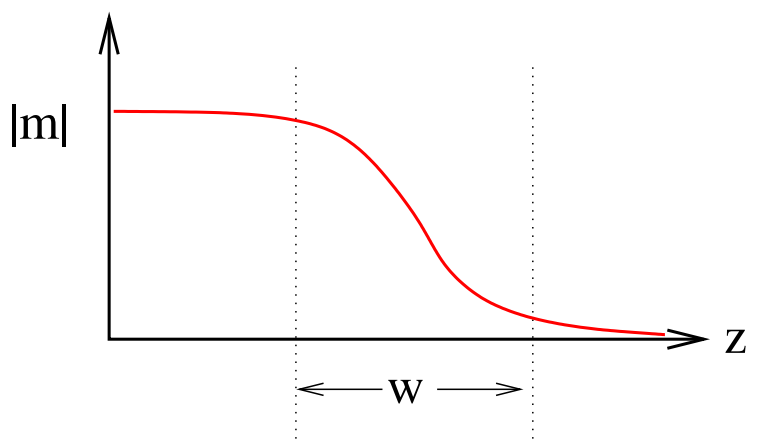

Figure 17: Spatially dependent fermion mass as a function of distance transverse to a bubble wall during a first-order EWPT.

We can rewrite the mass term in the form

$$
m(z)=|m| e^{i \theta(z) \gamma_{5}}
$$

imagining that the mass comes from the Higgs mechanism so that

$$
|m(z)|=y h(z)
$$

in the bubble wall, with some Yukawa coupling $y$. The $z$-dependence is illustrated in fig. 17. $\theta(z)$ is a $C P$-violating phase that arises from new physics.

One can solve the Dirac equation associated with (3.51), in an expansion in derivatives of $\theta$, and from that obtain the dispersion relation for the fermion in the background Higgs field. An eigenstate of energy has a spatially varying momentum: since the particle mass increases as it goes into the broken phase, its momentum must decrease to conserve energy. This gives a $C P$-conserving force on the particle, $F=\dot{p}$. But at first order in $\theta$ one finds a $C P$-violating component in the force, that acts oppositely on left- versus right-handed states and particles versus antiparticles. The total force at this order is [75]

$$
F \cong-\frac{\left(m^{2}\right)^{\prime}}{2 E} \pm \frac{\left(m^{2} \theta^{\prime}\right)^{\prime}}{2 E^{2}}+O\left(\theta^{2}\right)
$$

where the first term is $C P$-conserving and the second is $C P$-violating.

The semiclasscial force can induce the $f_{C P}(z)$ asymmetry in the distribution function for the quark, which then biases sphaleron interactions. To calculate this effect, we need to put the effect of a force back into the Boltzmann equation. It comes via the Liouville operator on the left-hand-side,

$$
\left(\frac{d}{d t}+\vec{v} \cdot \vec{\nabla}+\frac{\vec{F}}{m} \cdot \vec{\nabla}_{p}\right) f=\mathscr{C}[f]
$$

This is a partial differential-integral equation which is intractable. We usually just take the first moment of it by integrating over momenta to get an equation for the particle densities. But in the present case that is not an adequate approximation because the particles are disturbed away from kinetic as well as chemical equilibrium by the forces in the bubble wall [74].

It turns out to be sufficient to perturb the distribution function around the equilibrium one by introducing a small $z$-dependent chemical potential $\mu(z)$, to keep track of the local number density, 
plus an extra perturbation $\delta f_{\text {kin }}$ whose exact form is unknown, but which is assumed to be an odd function of the $z$ component of the particle's velocity $v_{z}$ [75]. Then we can take one additional moment, by integrating over momentum weighted by $v_{z}$. This introduces an dependent variable called the velocity perturbation,

$$
u=\left\langle\frac{p_{z}}{E} \delta f_{\text {kin }}\right\rangle
$$

Therefore each fermion interacting with the wall has associated with it four dimensionless functions, $\xi_{ \pm}=\mu_{ \pm} / T$ and $u_{ \pm}$, labeled by helicity, and four first-order coupled diffusion equations, of the general form

$$
\begin{aligned}
K_{1} v_{w} \xi_{ \pm}^{\prime}+K_{2} v_{w} u_{ \pm}+u_{ \pm}^{\prime}-\sum_{i j k} \Gamma_{i j k}\left(\xi_{i}, \xi_{j}, \xi_{k}\right)=0 \\
K_{3} \xi_{ \pm}^{\prime}+K_{4} v_{w} u_{ \pm}^{\prime}+K_{5} v_{w} u_{ \pm}+\Gamma_{ \pm} \xi_{ \pm}= \pm S
\end{aligned}
$$

where we have linearized in the wall velocity $v_{w}$, and $K_{i}$ are functions of $|m| / T$ [76] (beware that I am not following the same numbering scheme for the $K_{i}$ here). The three-particle interactions are represented by $\Gamma_{i j k}\left(\xi_{i}, \xi_{j}, \xi_{k}\right)$ which is a rate times a linear combination of the appropriate chemical potentials. The $\Gamma_{ \pm}$term represents the helicity-flipping rate inside the bubble (and also outside, for species that have a bare mass term), which damps the $C P$ asymmetry. The source term $S$ is due to the classical force, and takes the form

$$
S=v_{w} K_{6}\left(m^{2} \theta^{\prime}\right)^{\prime}
$$

The network (3.57) should be solved numerically to get an accurate estimate, since the solutions tend to change sign in the vicinity of the wall, and large cancellations can occur in their local contributions to the rate of baryon production.

Once the $C P$ asymmetries of the left-handed quarks and leptons are known, we can find the rate of baryon violation by sphalerons by integrating

$$
\dot{n}_{B}=-\frac{3}{2} \frac{\Gamma_{\mathrm{sph}}}{T} \sum_{i=1}^{3}\left(3 \mu_{u_{L}}^{i}+3 \mu_{u_{L}}^{i}+\mu_{e_{L}}^{i}+\mu_{v_{L}}^{i}\right)
$$

where the sum is over generations, and the factor $3 / 2$ is actually an approximation for a more complicated number, that is larger by a factor of 1.097 [77]. The baryon density is then

$$
n_{b}=\int d t \dot{n}_{B}=\int_{-\infty}^{\infty} \frac{d z}{v_{w}} \dot{n}_{B} \cong \int_{0}^{\infty} \frac{d z}{v_{w}} \dot{n}_{B}
$$

by changing variables from time to space via wall trajectory $z=v_{w} t$. The last approximation takes the sphaleron rate to vanish inside the bubble (the bubble is so large by this time that it is well-approximated as being planar, and we take $z<0$ to be the interior region) and the wall to be infinitesimally thin, but it is better to weight the integral by the local sphaleron rate, that smoothly interpolates to its value in the broken phase [78], and integrate over both regions.

\subsubsection{Getting a first-order EWPT}

So far we have described the means of violating $C P$, but we still need to fulfill the out-ofequilibrium condition for $\Gamma_{\text {sph }}$ inside the bubbles. One possibility that I will not discuss here is 

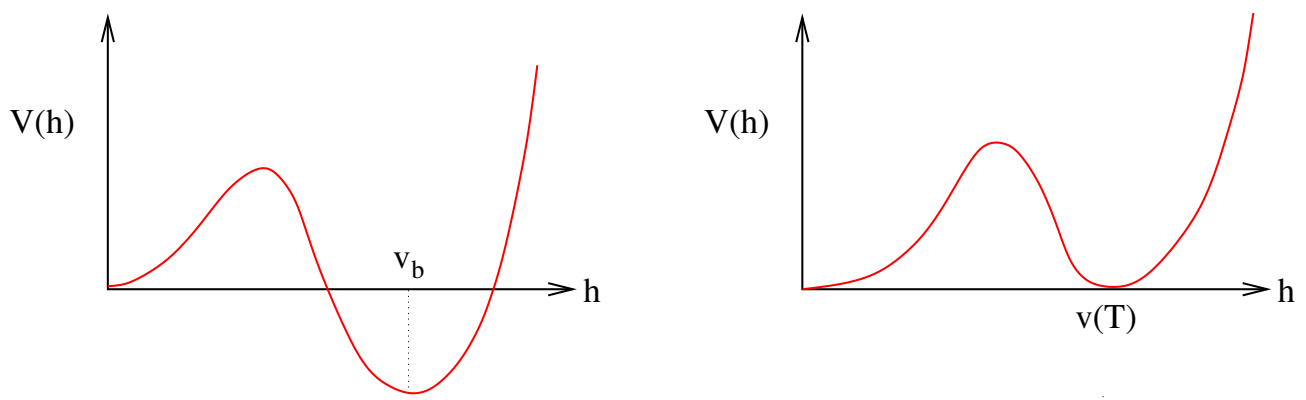

Figure 18: Left: form of the Higgs potential at the nucleation temperature $T_{n}$, consistent with geting a first-order phase transition. Right: the corresponding picture at the critical temperature $T_{c}>T_{n}$.

known as "cold electroweak baryogenesis," where the Higgs and gauge fields are far from equilibrium during preheating after inflation, and the reheat temperature is below the electroweak scale [79]. Instead I will assume a high reheat temperature. Then the out-of-equilibrium criterion relies upon the EWPT being first order. In the simplest models, the new physics should make the Higgs potential look like fig. 18 at high temperatures, with a barrier separating the $h=0$ (unbroken) and $h=v_{b}$ (broken) phases. The criterion for baryon number to not relax back too much toward zero inside the bubbles is [80]

$$
\frac{v_{b}}{T_{b}} \gtrsim 1.09
$$

where $T_{b}$ is the temperature at which baryogenesis takes place. Typically $T_{b}=T_{n}$, the nucleation temperature of the bubbles, which is lower than the critical temperature $T_{c}$ where the two vacua are degenerate. To determine $T_{n}$ one must find the rate per unit volume of bubble nucleation and equate it to $H^{4}[81,82]$.

The traditional method of generating the barrier in the potential was through finite-temperature effects from the "one-loop" thermal correction to the potential,

$$
V_{T}(h)=T \sum_{i} \pm \int \frac{d^{3} p}{(2 \pi)^{3}} \ln \left(1 \mp e^{-\beta \sqrt{p^{2}+m_{i}^{2}(h)}}\right) \quad\left(\begin{array}{c}
\text { bosons } \\
\text { fermions }
\end{array}\right)
$$

summed over all particles whose masses depend upon the Higgs field $h$ (here $\beta=1 / T$ ). This expression can be easily derived from statistical mechanics by recalling that the free energy is $F=-T \ln Z$ and the partition function is

$$
Z=\prod_{\vec{n}} \sum_{j=0}^{N}\left(e^{-\beta E(\vec{n})}\right)^{j}, \quad N=\left\{\begin{array}{c}
1, \text { fermion } \\
\infty, \text { boson }
\end{array}\right.
$$

and replacing the sum over modes that arises after taking the logarithm by $\sum_{\vec{n}} \rightarrow \int d^{3} n=L^{3} \int d^{3} p /(2 \pi)^{3}$ in a box of volume $L^{3}$.

At high temperatures, one can expand $V_{T}$ in powers of $m_{i}^{2} / T^{2}$. The contribution from bosons goes as

$$
V_{T}=\sum_{i} n_{i}\left(\frac{T^{2}}{24} m_{i}^{2}(h)-\frac{T}{12 \pi}\left(m_{i}^{2}(h)\right)^{3 / 2}+O\left(m_{i}^{4}\right)\right)
$$

where $n_{i}$ counts the number of degrees of freedom (e.g., 3 polarizations for a massive gauge boson) and the cubic term is the single nonanalytic contribution in the expansion. It plays the crucial role 
of generating the barrier if the particle gets all of its mass from the Higgs mechanism, $m_{i}^{2}=\frac{1}{4} g^{2} h^{2}$ for example, like the $\mathrm{SU}(2)_{L}$ gauge bosons. Then [83]

$$
V(h) \cong\left(\frac{n}{24} g^{2} T^{2}-\frac{1}{2} \lambda v^{2}\right) h^{2}-\frac{n g^{3}}{96 \pi} T h^{3}+\frac{\lambda}{4} h^{4}
$$

At the critical temperature $T_{c}$, pictured in fig. 18(right), this takes the form

$$
V(h)=\frac{\lambda}{4} h^{2}\left(h-v_{c}\right)^{2}
$$

where

$$
v_{c}=\frac{n g^{3}}{48 \pi \lambda} T_{c}
$$

Using the SM values $\lambda=0.13, g=0.65$ and $n=3 \times 3$ for the 3 massive vector bosons (approximating $g^{\prime}=0$ for simplicity), we find $v_{c} / T_{c}=0.13$, well below the bound (3.61), given that the ratio does not change very much between $T_{c}$ and $T_{n}$. We would need $\lambda$ and consequently $m_{h}$ to be smaller, $m_{h}<43 \mathrm{GeV}$, to satisfy the sphaleron bound. Lattice studies show that in fact for $m_{h}>80 \mathrm{GeV}$, there is no first order transition at all, but rather a smooth crossover [84].

Therefore new particles coupling to the Higgs are needed to enhance the strength of the EWPT. Notice that if $m_{i}^{2}(h)=m_{0}^{2}+g^{2} h^{2}$ with a bare mass term $m_{0}$ not coming from electroweak symmetry breaking (EWSB), then the "cubic" term is not really cubic and this reduces its effectiveness. It is difficult to add new particles with $m_{0}=0$ since they cannot be much heavier than the weak scale while remaining perturbatively coupled. Moreover, resumming the temperature corrections tends to generate $m_{0} \sim g T$ making this an even more generic problem.

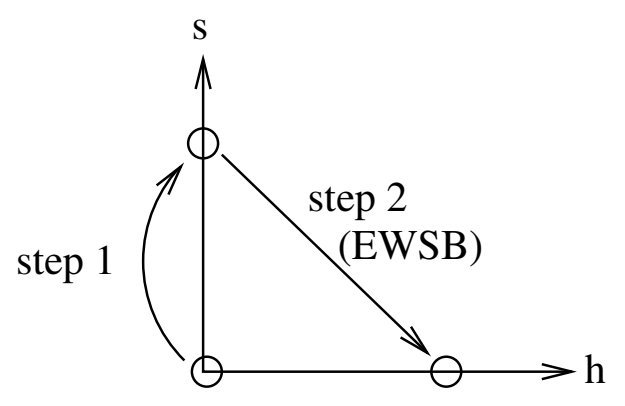

Figure 19: A two-step phase transition that can have a large tree-level barrier between the false vacuum $h=0$ and the true one where electroweak symmetry is broken.

A more robust way of strengthening the EWPT is to imagine a two-step phase transition involving a second field $S$ that is a singlet under $\mathrm{SU}(2)_{L}$. The sequence of symmetry breaking is shown in fig. 19: at very high temperature both $S=0$ and $h=0$; as $T$ decreases $S$ gets a VEV, and finally the electroweak transition occurs (during which $S$ may go to zero, but this is not strictly necessary). The tree-level potential is

$$
V=V_{S M}+\lambda_{s}\left(s^{2}-w^{2}\right)^{2}+\lambda_{h s} h^{2} s^{2}
$$

where the last term provides a barrier between the $h=0$ false minimum and the EWSB true minimum. This setup has the advantage that the barrier is already present at tree level and does not 
rely upon feeble finite-temperature effects that are suppressed by $g^{2} / 12 \pi$. One needs a moderate tuning to make the two minimum somewhat degenerate so that the finite temperature effect is just to reverse the relative heights of the two vacua. In this way one can relatively easily engineer a strong phase transition [85].

\subsubsection{The wall velocity}

I would like to finish this lecture by commenting upon an outstanding issue that is typically treated in a rough way because it is difficult to compute from first principles: the bubble wall velocity $v_{w}$. The predicted baryon asymmetry can be somewhat sensitive to its value, especially if it becomes too large. If $v_{w}>c / \sqrt{3}$, the sound velocity in the plasma, then baryogenesis essentially shuts off because there is no time for a $C P$ asymmetry to diffuse in front of the wall. The support of the integral (3.60) goes to zero. This behavior is not evident from the diffusion equations as written in (3.57) since they are linearized in $v_{w}$, and to my knowledge nobody has tried to quantify this for fast-moving walls. Such fast walls arise if the phase transition becomes very strong, with large supercooling $\left(T_{n} \ll T_{c}\right)$, which is an interesting limit because it leads to observable gravity waves [86]. Typically bubbles that give observable gravity waves are not compatible with baryogenesis, but exceptions can be found [87].

In a vacuum, an expanding bubble wall would quickly accelerate to the speed of light. To compute the wall velocity in a plasma, one must balance the outward force on the wall, due to the energy difference between the two vacua, against the force of friction from particles interacting with the wall. It requires self-consistently determining the shape of the wall at the same time, which is numerically challenging, since a stationary solution for the wall only exists if one guesses the right value of $v_{w}$. Most studies of EWBG therefore treat $v_{w}$ as a free parameter. It has been computed for the SM [88] (before $m_{h}$ was known to be too heavy for a first order EWPT) and the MSSM [89], typically giving small values $v_{w} \cong(0.05-0.1) c$.

\subsection{Exercises}

1. Out of equilibrium decay. Carry out the integration of the Boltzmann equation for the toy model of baryogenesis from out-of-equilibrium decay of an $X$ boson, with $C P$ asymmetry $\varepsilon$ between decays $X \rightarrow \bar{\Psi} \chi$ versus $X \rightarrow \Psi \bar{\chi}$, to evaluate the "washout" contribution from the inverse decays. In an exact treatment, the baryon number generated should go to zero as $H\left(m_{X}\right) / \Gamma_{X} \rightarrow 0$. Why does the present simplified approach go wrong in this respect?

2. Cut diagrams. (a) Using the Cutkosky rule for the internal propagators, compute the imaginary part of the self-energy $\Sigma_{I}$ at one loop for a heavy neutrino $N_{i}$ due to exchange of the Higgs boson and a lepton doublet $L_{j}$, considering them to be massless compared to $M_{i}$. You should find that $\Sigma_{I} \sim i \not p P_{R}$ where $p$ is the 4-momentum of the decaying $N_{i}$ (feel free to work in its rest frame) and $P_{R}=\left(1+\gamma_{5}\right) / 2$ is the chiral projection operator onto right-handed states.

(b) Compare the magnitude of $\Sigma_{I}$ to the tree-level decay rate of $N_{i}$. How does it appear in the correction to the propagator?

(c) If you never did a similar exercise before, compute the imaginary part directly from the iq prescription in the full propagators. Do the loop integral first; the imaginary part can be found from 
the Feynman parameter integral, using the fact that $\ln (z)$ has a branch cut along the negative $z$ axis, and is $\pm i \pi$ just above or below the cut.

3. $C P$ asymmetry from decays. Compute the part of the $\mathrm{CP}$ asymmetry $\varepsilon_{i}$ for leptogenesis coming from the interference between the tree and one-loop self-energy diagrams. The following steps will help.

(a) Using charge conjugation, one can show that

$$
\bar{L}_{i} \tilde{H} P_{R} N_{j}=\bar{N}_{j} \tilde{H}^{T} P_{R} L_{i}^{c}, \quad \bar{N}_{j} \tilde{H}^{\dagger} P_{L} L_{i}=\bar{L}_{i}^{c} \tilde{H}^{*} P_{L} N_{j}
$$

where $P_{L}=\left(1-\gamma_{5}\right) / 2$ and $L^{c}$ is the charge conjugated lepton doublet. We have used that $N=N^{c}$ since $N$ is a Majorana particle. This provides a convenient way of writing down the amplitudes with the lepton number flow going the "wrong" way, i.e., the tree versus loop diagrams. Write the amplitudes for the tree-level decay $N_{j} \rightarrow H^{*} L_{i}$ and from the one-loop $N$ self-energy diagram. (Why do we not care about the one-loop $L$ self-energy diagram?) As a check, your loop contribution should pick out the $M_{k} /\left(\left(\not p+\Sigma_{I}(p)\right)^{2}-M_{k}^{2}\right)$ part of the virtual $N_{k}$ propagator. Note that we included the one-loop self-energy to regulate the IR-divergence in case $p^{2} \cong M_{k}^{2}$, and used the fact that it goes like $\not p$ from problem 2 .

(b) Use the result of problem 2 to evaluate the imaginary part of the loop diagram. The $P_{R}$ in $\Sigma_{I}$ can be simplified since it is acting on a right-handed external state.

(c) Square the amplitude to find the imaginary part, and divide by the tree-level result to obtain $\varepsilon$ in terms of the Yukawa couplings, mass-squared differences, and decay rates $\Gamma_{i}$ as in our discussion of resonant leptogenesis. The $\gamma_{0}$ (or $\not p$ ) acting on the spinor for the decaying $N$ from the loop diagram can be disposed of using the Dirac equation for $N$ in its rest frame (or any frame).

4. Affleck-Dine baryogenesis [90]. ${ }^{4}$ Suppose $\phi$ is a complex field that carries baryon number 1, with Lagrangian

$$
|\partial \phi|^{2}-m^{2}|\phi|^{2}-i \lambda\left(\phi^{4}-\phi^{* 4}\right)
$$

The small quartic interaction violates baryon number, but not CP. (We can define the action of $C P$ on $\phi$ as $\phi \rightarrow e^{i \pi / 4} \phi^{*}$.) Instead, $C P$ is "spontaneously" broken by the initial condition of the field, $\phi=i \phi_{0}$ (purely imaginary) and $\dot{\phi}=0$.

(a) Write the equation of motion for $\phi$, assuming that the solution is spatially homogeneous.

(b) Let $\phi=\phi_{1}+i \phi_{2}$, and suppose that $\lambda \phi_{0}^{2} \ll m^{2}$ so that the interaction term is a small perturbation, and to zeroth order can be neglected, in particular for $\phi_{2}$ because at $t=t_{0}$ the imaginary part of the interaction term vanishes in the equation of motion. Then one can just solve the free field equation in an expanding background. Use the results you found in problem 1 of set 1 to construct the solution for $\phi_{2}$ that satisfies the initial condition at $t=t_{0}$, assuming that $m \gg H$ and the universe is radiation dominated.

(c) At $t=t_{0}$ the interaction term for $\phi_{1}$ does not vanish, so we expect the amplitude of $\phi_{1}$ to be of order $\lambda \phi_{0}^{3}$. But at late times, this inhomogeneous source term dies away due to Hubble damping, and we can again solve the noninteracting equation. Thus we can also estimate $\phi_{1}$ using problem 1 of set 1 . However there is in general a phase difference $\delta$ between $\phi_{1}$ and $\phi_{2}$ due to the early-time

\footnotetext{
${ }^{4}$ Beware of egregious typos if you read the paper.
} 
evolution, that turns out to be of order 1 (after solving numerically). Write down the appropriate estimate for $\phi_{1}$.

(d) The baryon number current is given by

$$
J_{B}^{\mu}=-i \phi^{*} \stackrel{\leftrightarrow}{\partial^{\mu}} \phi
$$

and the baryon number density is $n_{B}=J_{B}^{0}$. Show that initially $n_{B}=0$, but at late times it is nonzero. How does the out-of-equilibrium requirement come in?

\section{Dark Matter}

As mentioned in the introduction, many aspects of dark matter were treated by other lecturers at this school. My focus will be to make you aware of different classes of dark matter models, that can be distinguished by their respective production mechanisms. Thermal freezeout was covered in the lectures of P. Fox, but I will add some complementary observations, before going on to briefly discuss asymmetric dark matter, freeze-in, primordial black holes, and ultralight scalars/axions. But first, a little more history.

\subsection{Brief history of dark matter}

Often the history of science as recounted by its practitioners is oversimplified, since this results in a better story, or at least one that is easier to tell. I will do the same thing, claiming that in the case of dark matter it is really fair to credit Fritz Zwicky with originating the idea in his 1933 paper [91], where he also coined the term, in German. See ref. [92] for the unsimplified story. Oort is sometimes given precedence [93], based on studies of stellar dynamics around the Milky Way disk, but what he discovered is now known to be nonluminous baryons rather than dark matter.

Neither the term nor the concept caught on very quickly. Zwicky used it again, this time in English, in his 1937 paper [94], where he also proposed gravitational lensing as a way of mapping the dark matter distribution! However the term does not seem to appear again in the literature until 1979 in the review of Faber and Gallagher [95], which was also the most influential paper to start citing Zwicky's original 1933 work. Before that, "missing mass" or "missing matter" were in use. By this time the concept was established thanks to the galactic rotation curves measured by V. Rubin and collaborators [96] around 1970, although H. Babcock had already made a convincing such measurement for the Andromeda galaxy in 1939 [97]!

From the particle physics perspective, some highlights in the history of dark matter were:

- 1980: de Rujula and Glashow proposed massive neutrinos $\left(m_{v} \sim 24 \mathrm{eV}\right)$ as a dark matter candidate [98].

- 1983: White, Frenk and Davis showed that neutrino dark matter (with mass of order $30 \mathrm{eV}$ or less) erases cosmological structure on short distance scales, corresponding to the streaming length, predicting large voids that are not observed [99]. The idea that dark matter must be cold was thus born: DM should be nonrelativistic by the time the particle horizon contains a mass comparable to a galaxy. 
- 1984: Steigman and Turner coined the term "weakly interacting massive particle" (WIMP) [100], which became a popular paradigm for dark matter; but they had in mind decaying particles rather than dark matter. (Already in 1977, Lee and Weinberg had shown that heavy neutrinos annihilating with a weak scale cross section would overclose the universe if their masses were less than about $2 \mathrm{GeV}[101])$.

- 1985: Goodman and Witten adapted an idea of Drukier and Stodolsky (for detecting MeVscale neutrinos) [102] to the detection of weakly interacting DM particles [103]. Among the DM candidates they mentioned are axions, magnetic monopoles, sneutrinos, photinos, and exotic QCD bound states (which seems to have also influenced the list of candidates mentioned in [2]), reminding us of a saying, plus ça change, plus c'est la même chose, except for the magnetic monopoles, not currently a popular candidate.

Although hot dark matter has long been ruled out, the intermediate possibility of warm dark matter (WDM) is less clear. The canonical example is a sterile neutrino that was in thermal equilibrium, and becomes nonrelativistic at $T \sim m \sim 1 \mathrm{keV}$. This is the temperature at which $\rho H^{-3} \sim 10^{12} M_{\odot} \sim$ mass of the Milky Way. Lighter DM would erase galaxy-sized structures and be classified as hot. WDM with mass at the $\mathrm{keV}$ scale has a streaming length of $\sim 0.3 \mathrm{Mpc}$ [104].

Warm dark matter can help to address some problems of the cold dark matter scenario from $N$-body gravitational simulations, that give central density profiles of DM halos going as [105]

$$
\rho(r) \sim \frac{1}{r}
$$

This is too cuspy compared to observations of rotation curves, and is known as the cusp-core problem. A further problem is that CDM simulations predict too many dwarf satellite galaxies orbiting Milky Way-like halos (the "missing satellites" problem), and also too many highly luminous secondary galaxies (the "too big to fail" problem); for reviews see ref. [106, 107]. The origin of globular clusters is also mysterious within CDM.

But WDM does not seem to solve all problems simultaneously, giving too much suppression of satellites at a mass $\sim 2 \mathrm{keV}$ that would solve the cusp/core problem. Moreover, damping of power in the matter fluctuations at short scales would suppress Lyman- $\alpha$ absorption, leading to the recent constraint $m>3.5 \mathrm{keV}$ [108]. Thus WDM is becoming increasingly cold, and less motivated by an ability to address the small scale structure problems.

\subsection{Thermal freezeout}

The most popular mechanism for DM to attain its relic density is thermal freezeout, $\chi \chi \rightarrow f \bar{f}$ annihilations into SM particles, going out of equilibrium at a freezeout temperature quantified by $x_{f}=m_{\chi} / T_{f} \cong 16,28,30$ for $m_{\chi}=0.1 \mathrm{GeV}, 100 \mathrm{GeV}, 100 \mathrm{TeV}$. It is a fairly generic mechanism, assuming that the DM was in thermal equilibrium at early times. It works for self-conjugate DM as well as DM with a conserved charge, in which case $\chi \bar{\chi} \rightarrow f \bar{f}$ is the relevant interaction, and it is assumed there is no significant asymmetry between $\chi$ and $\bar{\chi}$ abundances (this is the subject of asymmetric dark matter, below). 
The basic result of thermal freezeout is that the relic density scales as

$$
Y_{\chi} \sim \frac{1}{\langle\sigma v\rangle m_{\chi} m_{p}}
$$

where $\langle\sigma v\rangle$ is the thermally averaged annihilation cross section times relative velocity. The corresponding annihilation rate is

$$
\Gamma=n_{\chi}\langle\sigma v\rangle
$$

and the annihilations go out of equilibrium at a temperature corresponding to

$$
\Gamma \sim H \sim \frac{T^{2}}{M_{p}}
$$

Hence

$$
Y_{\chi}=\frac{n_{\chi}}{s} \sim \frac{H}{\langle\sigma v\rangle s} \sim \frac{1}{\langle\sigma v\rangle M_{p} T}
$$

to be evaluated at the freezeout temperature $T_{f}$. To determine $T_{f}$ we recall that the particle density goes as $n_{\chi} \sim\left(m_{\chi} T\right)^{3 / 2} e^{-m_{\chi} / T}$ before it falls out of equilibrium. Then eqs. $(4.3,4.4)$ lead to the estimate

$$
x_{f} \equiv \frac{m_{\chi}}{T_{f}} \sim \ln \left(M_{p} m_{\chi}\langle\sigma v\rangle\right)-\frac{1}{2} \ln \left(x_{f}\right)
$$

which can be solved by iteration. This explains the logarithmic dependence on $m_{\chi}$ of $x_{f} \sim 16-29$ alluded to above, and our improved estimate

$$
Y_{\chi} \sim \frac{x_{f}}{\langle\sigma v\rangle M_{p} m_{\chi}}
$$

This refines the rougher estimate (4.2) by a number $x_{f} \sim 20$.

Now let's compare this prediction with observation. The Planck collaboration determines

$$
\Omega_{\mathrm{CDM}}=\frac{\rho_{\mathrm{CDM}}}{\rho_{\text {crit }}}=0.258
$$

while $\rho_{\mathrm{CDM}}=m_{\chi} Y_{\chi} s$ and $s=2891 / \mathrm{cm}^{3}$. Solving for the cross section gives

$$
\langle\sigma v\rangle \sim 10^{-9} \mathrm{GeV}^{-2} \sim 10^{-26} \mathrm{~cm}^{3} / \mathrm{s}
$$

independently of $m_{\chi}$ (except for the log dependence in $x_{f}$ ). This is considered to be a typical weak scale cross section with $\sigma \sim \alpha^{2} /(100 \mathrm{GeV})^{2}$ and $\alpha \sim 10^{-2}$. Thus one seems to get the right relic density, independent of $m_{\chi}$ just by having new physics near the weak scale. This was called a "striking coincidence" in the review article [109]. Later it came to be known as the "WIMP miracle."

To be more quantitative, we need to solve the Boltzmann equation, similarly to the case of $X^{\prime}$ decays in our toy model of baryogenesis. We define $Y_{\chi}=n_{\chi} / s$ and assume $n_{\chi}=n_{\bar{\chi}}$ in case $\chi$ is not self-conjugate. As before $x=m_{\chi} / T$ takes the place of the time variable. Then

$$
\dot{n}_{\chi}+3 H n_{\chi}-\langle\sigma v\rangle\left(n_{\chi}^{2}-n_{\chi, e q}^{2}\right)
$$

\footnotetext{
${ }^{5}$ This term was introduced by Jonathan Feng at the SLAC Summer Institute lectures in 2001. He was motivated by a sense of frustration that particle theorists did not take the striking coincidence very seriously. His strategy seems to have worked. The name first appears in a research article in 2008 [110], when it was already in common use.
} 
becomes

$$
\frac{d Y_{\chi}}{d x}=-\frac{x s\langle\sigma v\rangle}{H\left(m_{\chi}\right)}\left(Y_{\chi}^{2}-Y_{\mathrm{eq}}^{2}\right)
$$

where

$$
Y_{\mathrm{eq}}=\frac{45}{4 \pi^{4}} \frac{g_{\chi}}{g_{*, s}} x^{2} K_{2}(x) \cong \frac{45}{2 \pi^{4}}\left(\frac{\pi}{8}\right)^{1 / 2} \frac{g_{\chi}}{g_{*, s}} x^{3 / 2} e^{-x}
$$

for a nonrelativistic particle with $g_{\chi}$ degrees of freedom ( 1 for a real scalar, 2 for a complex scalar or Weyl fermion, 4 for a Dirac fermion), $g_{*, s}$ was defined in eq. (3.18), the entropy density is

$$
s=\frac{\rho+p}{T} \cong 0.44 g_{*, s} \frac{m_{\chi}^{3}}{x^{3}}
$$

and the Hubble parameter

$$
H=1.66 \sqrt{g_{*}} \frac{T^{2}}{M_{p}}
$$

is evaluated at $T=m_{\chi}$, with $g_{*}$ defined similarly to (3.18), but with $\left(T_{i} / T\right)^{4}$ instead of $\left(T_{i} / T\right)^{3}$, such that the energy density is $\rho=\left(\pi^{2} / 30\right) g_{*} T^{4}$.

The cross section is thermally averaged, and the $v$ appearing there is usually considered to be the relative velocity between the annihilating particles, but there is some subtlety in this identification that becomes relevant when the annihilations are relativistic [111], which is not the case here. Since the particles are highly nonrelativistic for thermal freezeout, it is a good approximation to use Maxwell-Boltzmann statistics so that

$$
\langle\sigma v\rangle \sim \int d^{3} p_{1} d^{3} p_{2} e^{-E_{1} / T-E_{2} / T} \sigma\left|\vec{v}_{1}-\vec{v}_{2}\right|
$$

A proper treatment of $v$ in [112] allows this to be expressed as a single integral over the Mandelstam $s=\left(p_{1}+p_{2}\right)^{2}\left(=4 E^{2}\right.$ in the center-of-mass frame $)$,

$$
\langle\sigma v\rangle=\frac{1}{8 m^{4} T K_{2}^{2}(m / T)} \int_{4 m^{2}}^{\infty} d s \sqrt{s}\left(s-4 m^{2}\right) K_{1}(\sqrt{s} / T) \sigma(s)
$$
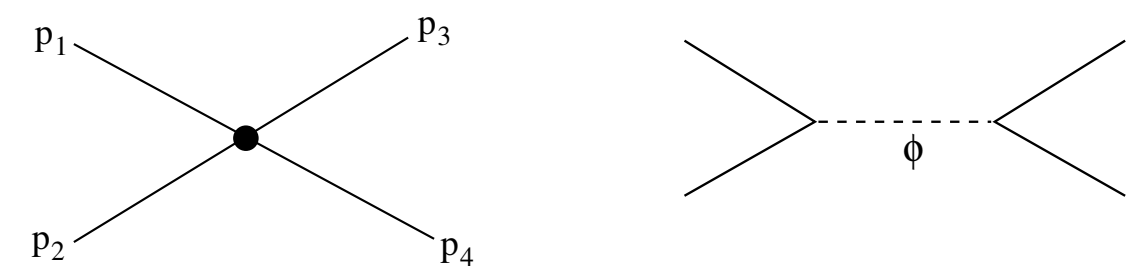

Figure 20: Left: generic annihilation of dark matter particles ( 1 and 2$)$ into standard model particles ( 3 and 4). Right: resonantly enhanced annihilation.

For purposes of estimation it is often adequate (if one does not insist on great precision) to ignore the thermal averaging and just compute $\sigma v$ in the limit $v \rightarrow 0$, since for $s$-wave annihilation this is just a constant. Then for the case of DM annihilating into equal mass particles, with particles labeled as $1+2 \rightarrow 3+4$ as in fig. 20(right),

$$
\sigma=\int_{t_{0}}^{t_{1}} d t \frac{|\mathscr{M}|^{2}}{64 \pi s p_{1, \mathrm{~cm}}^{2}} \cong \frac{|\mathscr{M}|^{2} p_{3, \mathrm{~cm}}}{16 \pi s p_{1, \mathrm{~cm}}}
$$


in terms of the matrix element $\mathscr{M}$, the center of mass momenta of particles 1 and 3 , and the integration limits

$$
t_{0,1}=-\left(p_{1, \mathrm{~cm}} \pm p_{3, \mathrm{~cm}}\right)^{2}
$$

See the kinematics review of [1]. Taking $v_{\text {rel }}=2 p_{1, \mathrm{~cm}} / E=2 \sqrt{1-4 m^{2} / s} \cong 2 p_{1, \mathrm{~cm}} / \mathrm{m}, s \cong 4 m^{2}$ and $p_{3, \mathrm{~cm}}=\sqrt{1-m_{3}^{2} / m^{2}}$, we find

$$
\sigma v_{\text {rel }} \cong \frac{|\mathscr{M}|^{2}}{32 \pi m^{2}} \sqrt{1-m_{3}^{2} / m^{2}}
$$

(times $1 / 2$ if particles 3 and 4 are identical). For simplicity we have assumed that $m_{4}=m_{3}$, which is usually the case.

The relative correction from thermally averaging should be of order $v^{2} \sim T / m \sim 1 / 20$. Of course this does not work if the cross section is $p$-wave suppressed since then $\sigma v \rightarrow 0$ in the limit of $v \rightarrow 0$. Generally one can write

$$
\langle\sigma v\rangle \cong \sigma_{0}\left(\frac{T}{m}\right)^{n}
$$

in the low-temperature limit, with $n=0$ for $s$-wave annihilation, $n=1$ for $p$-wave, etc. Another case where the simple estimate fails is when the cross section is resonantly enhanced by an intermediate particle whose mass happens to be close to twice that of the DM, as in fig. 20(right). The thermal averaging is then imporant since it allows $s$ to vary near the pole of the propagator in

$$
\mathscr{M} \sim \frac{1}{s-m_{\phi}^{2}+i m_{\phi} \Gamma_{\phi}}
$$

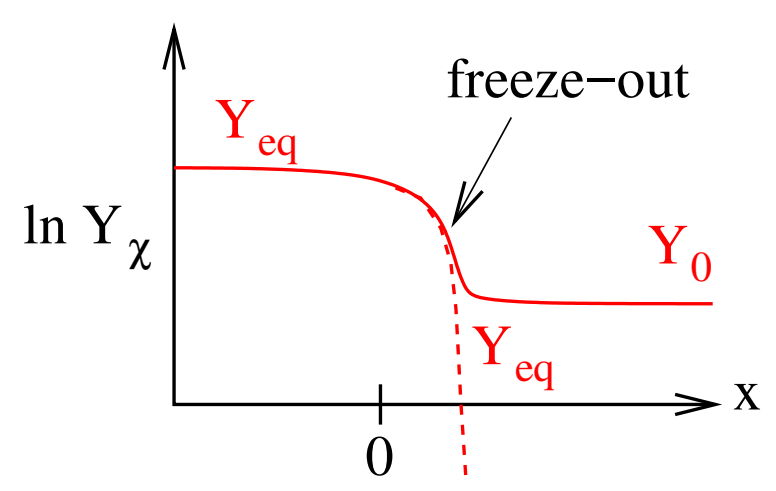

Figure 21: Solution of Boltzmann equation (solid curve), compared to the equilibrium abundance (dashed).

To find the relic density, one must integrate the Boltzmann equation (4.11), whose solution looks qualitatively like fig. 21. But it is numerically challenging to follow the whole evolution since the abundance changes very rapidly around the epoch of freezeout, so one often tries to avoid writing code from scratch. Publicly available codes like micrOmegas [114] and DarkSUSY [115] are commonly used.

For a fast estimate, in the case of $s$-wave annihilation, one can simply look up the value of the cross section for a given DM mass that yields the observed relic density, [113], reproduced in fig. 22. (An improved version of this result can be found in fig. 3 of [115].) The "canonical" value 


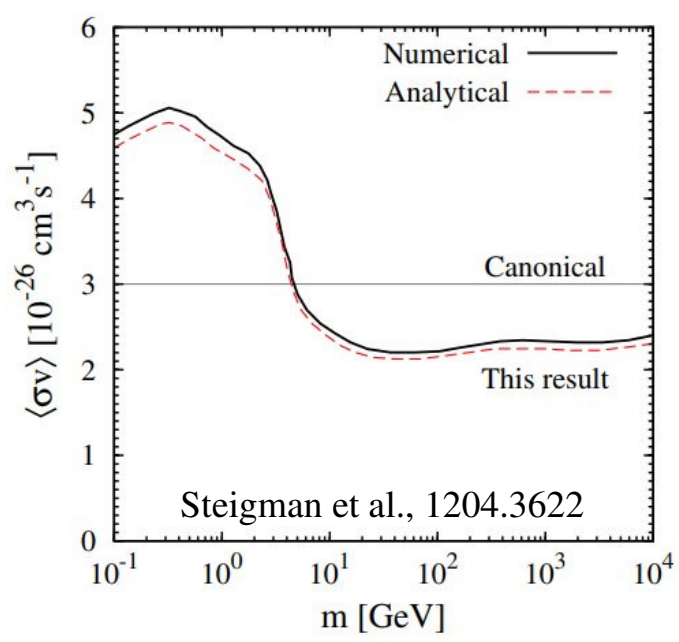

Figure 22: Value of $\sigma v$ needed to get the observed relic density, for Majorana or self-conjugate scalar DM, reproduced from ref. [113].

of $3 \times 10^{-26} \mathrm{~cm}^{3} / \mathrm{s} \cong 25 \times 10^{-10} \mathrm{GeV}^{-2}$ assumes self-conjugate DM, like a Majorana fermion or a real scalar. For Dirac or complex scalar DM, one should multiply this result by 2, as can be seen from comparing the rate of annihilation in the two cases. For self-conjugate DM, the rate of annihilation per $\chi$ particle is

$$
\Gamma=n_{\chi}\langle\sigma v\rangle
$$

leading to the density $n_{\chi} \sim H /\langle\sigma v\rangle$. But for Dirac or complex scalar DM, it is

$$
\Gamma=n_{\bar{\chi}}\langle\sigma v\rangle
$$

where now the total DM density is given by $n_{\chi}+n_{\bar{\chi}}$. The estimate $n_{\chi} \sim H /\langle\sigma v\rangle$ remains the same, but $\langle\sigma v\rangle$ must be doubled to get the same value for the total density. Notice that for Dirac DM, the total abundance is $Y_{\chi}+Y_{\bar{\chi}}$, so the same Boltzmann equation (4.11) can be used for Dirac or Majorana DM.

Finally, there is an approximate analytic solution to the Boltzmann equation [116], slightly improved upon in [117]. The present abundance can be estimated as

$$
Y_{0} \cong \sqrt{\frac{45 g_{*}}{\pi g_{* s}^{2}}} \frac{(n+1) x_{f}^{n+1}}{m_{\chi} M_{p} \sigma_{0}}
$$

with $n$ and $\sigma_{0}$ defined in eq. (4.20), and

$$
\begin{aligned}
& x_{f} \cong \ln y_{f}-\frac{1}{2} \ln \ln y_{f} \\
& y_{f}=\frac{g_{\chi}}{2 \pi^{3}} \sqrt{\frac{45}{8 g_{*}}} m_{\chi} M_{p}(n+1) \sigma_{0} ;
\end{aligned}
$$

compare with eq. (4.6). This approximation scheme is valid in principle for any DM mass, so long as freezeout occurs when $x_{f} \gg 1$ so the particle is nonrelativistic. But there is an upper limit on $m_{\chi}$ 
from partial wave unitarity [118],

$$
\langle\sigma v\rangle \lesssim \frac{4 \pi}{m_{\chi}^{2}}\left\langle\frac{1}{v_{\text {rel }}}\right\rangle
$$

assuming $s$-wave annihilation. One cannot get a large enough cross section to sufficiently suppress the relic density if $m_{\chi}$ becomes too large. Taking the modern value of $\Omega_{\mathrm{CDM}}$, this limit is $m_{\chi} \lesssim$ $140 \mathrm{TeV}$, assuming self-conjugate DM. Of course this bound does not apply if many partial waves contribute, which would be the case for composite DM made from weakly bound constituents, giving a geometrical cross section (see for example ref. [119]).

\subsection{Asymmetric dark matter (ADM)}

Although thermal freezeout seems like a generic mechanism, the fact that baryonic matter gets its abundance from the matter-antimatter asymmetry makes it quite reasonable that dark matter could have a similar origin, if it has a conserved number density analogous to baryon number. This is a large subject that cannot be done justice in the little time I have here; see ref. [120] a comprehensive review. I will not say anything about the specific mechanism for generating the dark asymmetry, but simply assume its existence.

Whereas the density of baryonic antimatter in the universe is negligible, this need not be the case in the dark sector, where there can be a significant symmetric component to the density in addition to the dominant asymmetric component. We can define the two as

$$
\begin{aligned}
n_{\text {sym }} & =n_{\chi}+n_{\bar{\chi}}-\left|n_{\chi}-n_{\bar{\chi}}\right|=\min \left(n_{\chi}, n_{\bar{\chi}}\right) \\
n_{\text {asym }} & =\left|n_{\chi}-n_{\bar{\chi}}\right| \cong \max \left(n_{\chi}, n_{\bar{\chi}}\right)
\end{aligned}
$$

For ADM, it is assumed that $n_{\text {sym }} \ll n_{\text {asym }}$. Then indirect detection signals from $\chi \bar{\chi} \rightarrow f \bar{f}$ in the galaxy will be suppressed relative to thermal DM. The question is, how much will the constraints be weakened? One has to solve the Boltzmann equation again, but now taking into account the conserved particle number in the asymmetric component.

The fact that $n_{\text {asym }}$ cannot be reduced by annihilations leads to qualitatively different results for $n_{\text {sym }}$ than in the case of thermal DM, where the annihilations turn off once the density falls below $n_{\text {eq }}$. For ADM, the density can no longer fall like $n_{\text {eq }}$ once it reaches $n_{\text {asym }}$. Therefore the annihilations continue longer than for thermal DM, and $n_{\text {sym }}$ becomes much smaller than the corresponding thermal relic density. This was worked out in ref. [121], with the result shown in fig. 23. The solid curve shows the ratio of abundances, $n_{\mathrm{sym}} / n_{\mathrm{asym}}$ as a function of the cross section, in units of the canonical value for thermal DM. The dashed line shows the resulting suppression factor in any indirect detection signal from annihilations at late times. Interestingly, for the case of $\chi \bar{\chi} \rightarrow e^{+} e^{-}$or other electromagnetically interacting final states, this leads to a lower limit on $\langle\sigma v\rangle$ in order to sufficiently suppress annihilations [122],

$$
\langle\sigma v\rangle \gtrsim \begin{cases}1 \times 10^{-25} \mathrm{~cm}^{3} / \mathrm{s}, & m_{\chi}=10 \mathrm{GeV} \\ 7 \times 10^{-25} \mathrm{~cm}^{3} / \mathrm{s}, & m_{\chi}=1 \mathrm{MeV}\end{cases}
$$

If the ADM is bosonic, there can be a different kind of indirect signal, from its accumulation in neutron stars. Bosons may achieve such a high central density that a black hole can form that will consume the neutron star. This gives a limit on the cross section for scattering on nucleons of $\sigma_{\chi N} \lesssim 10^{-47} \mathrm{~cm}^{2}$ in a range of masses $m \chi \in[5 \mathrm{MeV}-15 \mathrm{GeV}]$, where direct detection constraints are relatively weak. 


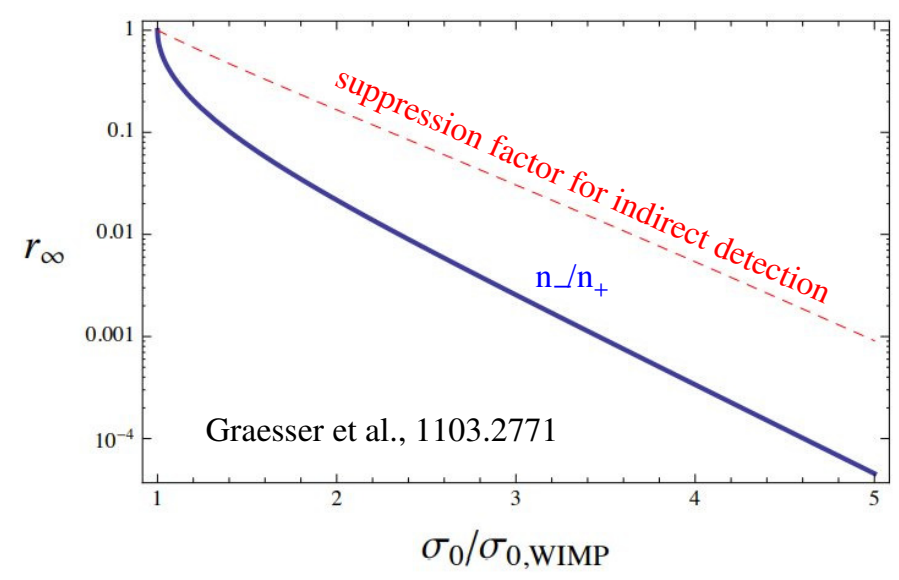

Figure 23: The suppression of the ratio of symmetric to asymmetric components of asymmetric dark matter as a function of annihilation cross section (solid curve) and the resulting suppression factor for indirect detection signals (dashed), from ref. [121].

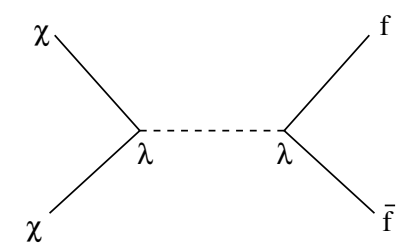

Figure 24: An annihilation process that is suppressed at high temperatures, where freeze-in may be taking place.

\subsection{Freeze-in}

It is possible that DM interacts so weakly that it never came into thermal equilibrium in the early universe. Then initially we would have $Y \cong 0$ and the Boltzmann equation would be approximately

$$
\frac{d Y_{\chi}}{d x} \cong \frac{x s\langle\sigma v\rangle}{H\left(m_{\chi}\right)} Y_{\mathrm{eq}}^{2}
$$

$Y_{\chi}$ will then slowly approach $Y_{\text {eq }}$ from below. We can estimate its present abundance by integrating eq. (4.29). Unlike for thermal freezeout, it may not be a good approximation to take $\langle\sigma v\rangle$ to be constant because the result, going as $\int d x x^{2} K_{2}^{2}(x)$, is dominated by $x \sim 0$, i.e., high temperatures, and there will generally be some temperature dependence in $\langle\sigma v\rangle$ at high $T$. For example, consider the process in fig. 24 with $s$-channel boson exchange, which gives $\langle\sigma v\rangle \sim \lambda^{4} x^{2} / m_{\chi}^{2}$. We find that

$$
m_{\chi} Y_{0} \sim 10^{-4} \lambda^{4} M_{p} \cong 4.3 \times 10^{-10} \mathrm{GeV}
$$

in order to get the observed relic density, requiring $\lambda \sim 10^{-6}$. This is a very weak coupling compared to that needed for thermal freezeout. To compare them, supposing $\langle\sigma v\rangle=\lambda^{4} / m_{\chi}^{2}$ as $T \rightarrow 0$, and the cross section needed for thermal freezeout is denoted by $\langle\sigma v\rangle_{0}$, then

$$
\frac{\langle\sigma v\rangle}{\langle\sigma v\rangle_{0}} \sim\left(\frac{0.1 \mathrm{eV}}{m_{\chi}}\right)^{2}
$$


showing that for any reasonably heavy DM, the freeze-in cross section is many orders of magnitude below that for freeze-out.

An interesting application of freeze-in is to DM candidates that have only gravitational interactions, since these must be present regardless of model-building choices [123, 124]. In this case $\langle\sigma v\rangle \sim T^{2} / M_{p}^{4}$ which leads to $\int d x K_{2}(x)^{2} \sim\left(T / m_{\chi}\right)^{3}$ : the integral is dominated by the hightemperature contribution, and is therefore sensitive to the reheat temperature after inflation. Dark matter of mass up to $\sim 10^{16} \mathrm{GeV}$ can have the right relic density. It is argued that even such heavy dark matter could have observable signatures, since quantum gravitational effects are believed to break any global symmetries [125], including those that might stabilize dark matter. Then, for example, fermionic DM could decay through the operator $\bar{\chi} H L$, just like a heavy sterile neutrino, suppressed by the action of a gravitational instanton via $e^{-S}$.

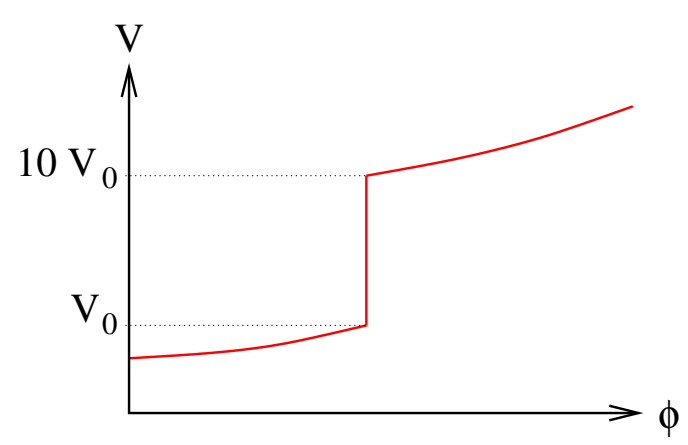

Figure 25: A step discontinuity in the inflaton potential, that enhances power in fluctuations at a specific scale. A factor of $\sim 10$ change in $V$ is required to get a factor of $\sim 10^{7}$ enhancement in the power.

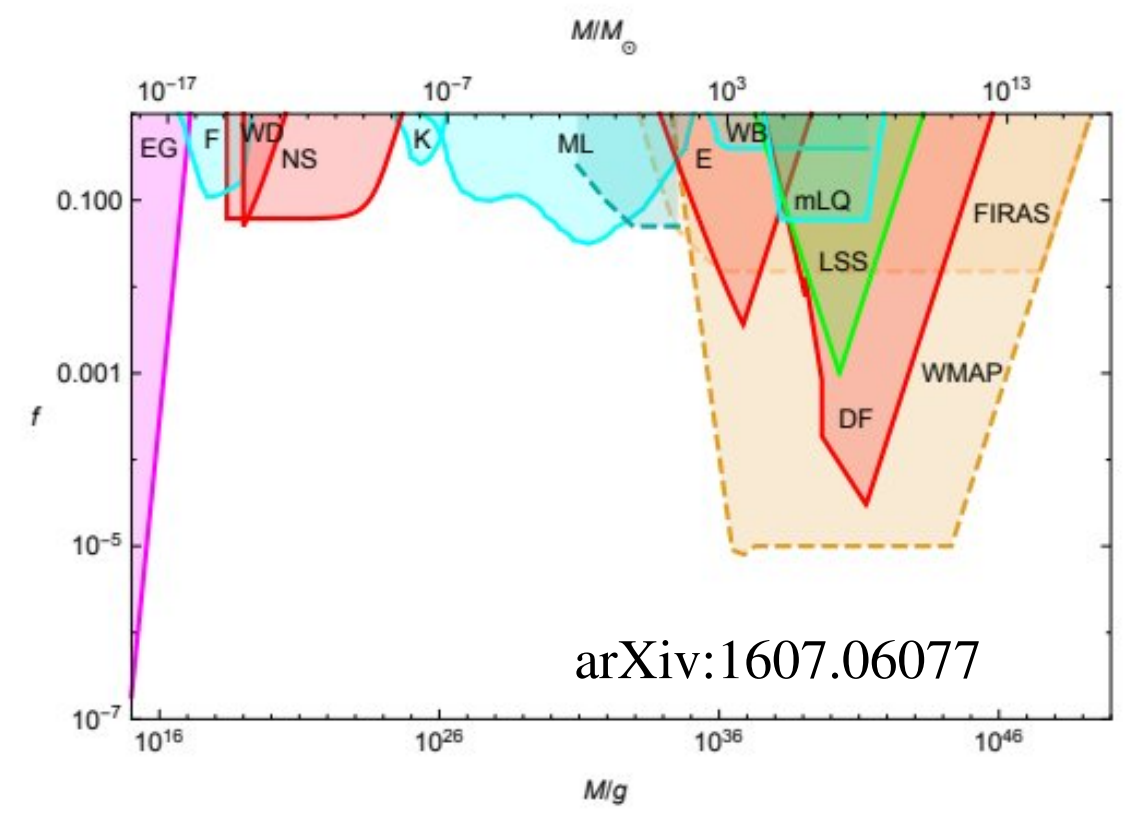

Figure 26: Upper limit on the fraction $f$ of DM in PBHs of a given mass, assuming monochromatic distribution of masses, from ref. [126]. 


\subsection{Primordial black holes}

The recent LIGO discovery of black hole mergers with masses of $\sim 30 M_{\odot}$ [127] has renewed interest in primordial black holes (PBHs) as dark matter. There are many constraints on PBH dark matter, which ostensibly rule them out as being all of the dark matter at any mass, assuming their masses are all the same (monochromatic mass function). Fig. 26 from ref. [126] shows the maximum fraction $f$ of the total dark matter that is allowed in PBHs of a given mass. Ref. [128] found $f \lesssim 0.1$ for $30 M_{\odot}$ PBHs, using FIRAS observations of the CMB spectral shape. This would have been distorted by X-rays from material accreting onto the PBHs. Since the LIGO discovery, this constraint was reconsidered and shown to be weaker in ref. [129], leaving room for PBHs below $100 M_{\odot}$. Complementary constraints from lack of disruption of a star cluster in Eridanus disfavor the $30 M_{\odot}$ mass region [130] but are subject to large uncertainties.

It is sometimes said that PBHs are a very conservative DM candidate because they require no new physics, but this statement ignores the new physics that is probably needed to produce PBHs with the observed relic density. Hybrid inflation can produce PBHs, but in a mass range far below the LIGO region [131]. A more exotic inflationary scenario seems to be needed in order to produce density fluctuations of sufficient power at wavelengths $\lambda$ associated with the desired mass scale (quantified below). A nearly scale-invariant spectrum extending to these scales (extrapolated from the COBE scale) has far too little power, since the fluctuations are presumed to be Gaussian and one needs a large amplitude $\delta \rho / \rho \sim 1$ to produce a black hole [132]. The simplest way to produce a spike in the power at a given scale is to introduce a sharp step in the inflaton potential [133] (see fig. 25), that the inflaton crosses during the late stages of inflation, at the moment when the scale $\lambda$ of interest first crosses the horizon.

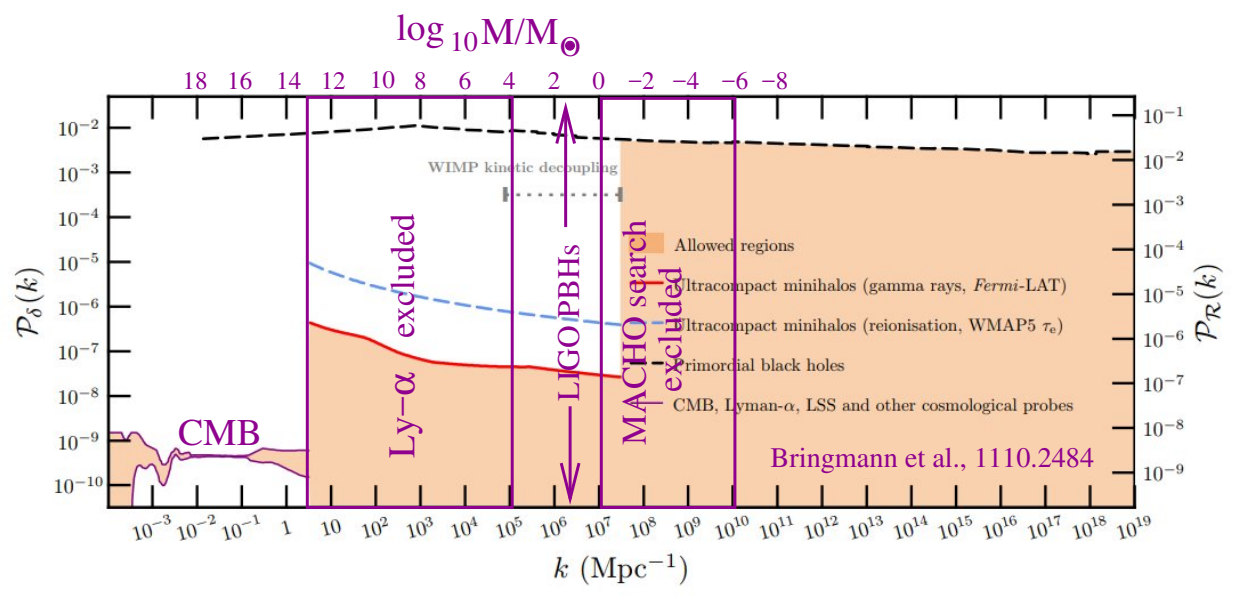

Figure 27: Constraints on power of $\delta \rho / \rho$ fluctuations versus wavenumber, adapted from ref. [134]. The corresponding black hole mass on the top scale is estimated as $M_{\mathrm{PBH}} \sim \rho_{\text {crit }} / k^{3}$. See [126] for a summary of excluded PBH mass ranges.

In particular to get PBHs to be all of the DM, we need a boost of order $10^{7}$ times in the power spectrum $\mathscr{P}_{\delta}$ of $\delta \rho / \rho$, at the scale $k \sim 10^{6} \mathrm{Mpc}^{-1}$, nearly a million times smaller than those probed by large scale structure, $\sim 3 \mathrm{Mpc}^{-1}$. The situation is illustrated in fig. 27 , based on ref. [134]. The dashed curve near the top shows the power needed to produce PBHs with the right relic density, 
concentrated at a given mass scale. (The shaded regions refer to constraints from ultracompact minihalos, which can be ignored in the present discussion.) I have overlaid on that figure a rough scale of PBH masses and a few constraints neighboring the LIGO mass region, compiled by ref. [126]).

The PBH mass corresponding to a fluctuation of physical wavelength $\lambda$ is the mass contained in a horizon volume when that scale crosses the horizon, $M_{\mathrm{PBH}} \sim \rho \lambda^{3} \sim \rho / H^{3}$. For most modes of interest, horizon crossing occurs during radiation domination, and so one must take into account the additional redshifting of density when relating this mass to comoving scales. The result is roughly [135]

$$
M_{\mathrm{PBH}} \sim 2 M_{H, \mathrm{eq}}\left(\frac{k_{\mathrm{eq}}}{k}\right)^{2} \cong 6 \times 10^{13} \frac{M_{\odot}}{(k \cdot 1 \mathrm{Mpc})^{2}}
$$

where $M_{H, \mathrm{eq}}=3.5 \times 10^{17} M_{\odot}$ is the horizon mass at equality and $k_{\mathrm{eq}}=0.01 \mathrm{Mpc}^{-1}$ is the corresponding wavenumber. The power of $1 / k$ is 2 instead of 3 because during radiation domination, $\rho$ scales as $k^{4}$ rather than $k^{3}$. Hence if the relevant scale crosses the horizon during radiation domination, there is an extra factor of $k$ to account for.

Fig. 27 demonstrates how fast the power in density fluctuations has to rise as a function of wave number, to grow from its small value well-constrained CMB region by seven orders of magnitude within six decades of $k$. A correspondingly sharp feature in the inflaton potential would be needed. Not only should the feature be in a special location, but the magnitude of the step (fig. 25) must be tuned very precisely to get the right relic density. This is because the density fluctuations are Gaussian, hence exponentially sensitive to the power. This is quantified in problem 4 below. From the theoretical viewpoint, this makes PBHs look like a peculiar dark matter candidate.

\subsection{Fuzzy or axion-like dark matter}

One of the earliest DM candidates, and still very popular, is a very light scalar field, the axion [136, 137], having a tilted wine-bottle potential (fig. 28),

$$
\mathscr{L}=\frac{1}{2} f^{2}(\partial a)^{2}-\Lambda^{4} \cos a
$$

giving it a mass

$$
m_{a}=\frac{\Lambda^{2}}{f}
$$

Notice that we have taken $a$ to be dimensionless here so it is an angular variable. The basic idea (see lectures of A. Hook, this school) is that the axion would have been a Goldstone boson of a spontaneous broken symmetry, but the symmetry is explicitly broken by nonperturbative (instanton) effects, at a scale $\Lambda$ that might be suppressed by a small tunneling probability. In the case of QCD there is no such suppression because large instantons correspond to large running couplings, with small tunneling actions, and the favored axion mass range is $m_{a} \in\left[10^{-6}, 10^{-2}\right] \mathrm{eV}$ [138].

On the other hand, string theory generically predicts many axion-like particles whose masses are exponentially suppressed [139], and could naturally be much lighter. An interesting mass scale for cosmology is $m \sim 10^{-22} \mathrm{eV}$, whose corresponding de Broglie wavelength $\lambda \sim \mathrm{kpc}$ coincides with the size of the central region of a Milky Way-like galaxy. Recall the core-cusp problem, discussed in section 4.1. Such a large $\lambda$ would prevent central cusps on this scale. The cusp is 
"fuzzed out," and the scenario is known as "fuzzy dark matter" [140]. Despite the small mass, it is another form of CDM since it is presumed to be too weakly coupled to have thermalized.

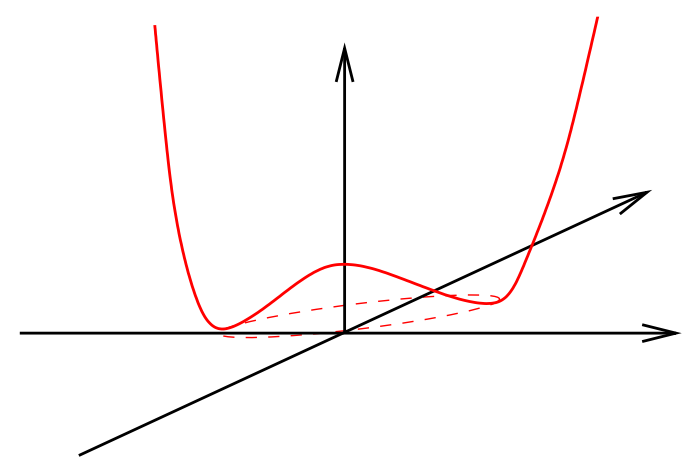

Figure 28: Potential for an axion-like DM candidate.

Axion-like particles (ALPs) can get their relic density from the misalignment mechanism, if the potential term $\Lambda^{4} \cos a$ is negligible at early times. For example for the QCD axion, this term is suppressed by powers of $T$ at high temperature [141]. Then during inflation, due to its quantum fluctuations, $a$ will take a random initial value $a_{i} \in[0,2 \pi]$, which becomes homogenous in a given causal patch during the inflationary expansion. As the universe cools, eventually the $\Lambda^{4} \cos a$ becomes important: as soon as $H$ falls below $m_{a}$, the axion starts oscillating around the minimum of the potential, with decaying amplitude

$$
a(t) \sim a_{i}\left(\frac{R_{i}}{R}\right)^{3 / 2}=a_{i}\left(\frac{T}{T_{i}}\right)^{3 / 2}
$$

Here $R$ is the scale factor, and $R_{i}$ its value when $H\left(T_{i}\right)=m_{a}$. At the corresponding temperature $T=T_{i}$, the energy density is of order $\rho_{a} \sim \Lambda^{4}$. Therefore the present density of axions is

$$
\begin{aligned}
\Omega_{a} \sim \frac{\Lambda^{4}}{\rho_{\text {crit }}}\left(\frac{T_{0}}{T_{i}}\right)^{3} & =0.28\left(\frac{m_{a}}{10^{-22} \mathrm{eV}}\right)^{1 / 2}\left(\frac{f}{4 \times 10^{16} \mathrm{GeV}}\right)^{2} \\
& =0.28\left(\frac{m_{a}}{10^{-3} \mathrm{eV}}\right)^{1 / 2}\left(\frac{f}{7 \times 10^{11} \mathrm{GeV}}\right)^{2}
\end{aligned}
$$

highlighting fiducial values corresponding to QCD-like axions and fuzzy DM, respectively.

An important caveat is that we assumed the approximate global symmetry was already broken at a scale above that of inflation. If the phase transition happens after inflation, then there can be important additional contributions to the relic density from the formation and decay of axion strings and domain walls. It is difficult to reliably quantify these extra contributions; simulations of the string network are needed. See for example ref. [142].

\subsection{Self-interacting dark matter (SIDM)}

It doesn't exactly fit into my general scheme of classifying DM models by their production mechanisms, but it is important to mention the possibility that dark matter may have strong selfinteractions [143], since this has become a popular approach to solving the small-scale structure problems of CDM that were discussed around eq. (4.1); see [144] for a review. 
SIDM can produce cored DM profiles as illustrated in fig. 29. An energetic DM particle in an elliptical orbit may scatter with a DM particle in the central region, giving it energy and allowing it to move out of the central region. Velocities are higher in the outer regions [145] so this provides a way of transferring energy to the lower-velocity particles that would otherwise be trapped near the center. This is confirmed by $N$-body simulations incorporating SIDM [146, 147]. Cross sections of order

$$
\frac{\sigma}{m} \sim 0.1 \frac{\mathrm{cm}^{2}}{\mathrm{~g}}=0.17 \frac{\mathrm{barn}}{\mathrm{GeV}}
$$

are found to solve the cusp-core and other small-scale structure problems, while being compatible with the Bullet Cluster constraint [148]. (For reference, nucleons in the real world have $\sigma / m \sim$ $20 \mathrm{~b} / \mathrm{GeV}$.) The combination $\sigma / m$ is relevant because the scattering rate is

$$
\Gamma=n \sigma v=\rho \frac{\sigma}{m} v
$$

and $\rho$ is fixed by $\Omega_{\mathrm{CDM}}$.

It is natural to expect self-interactions if the DM is part of a larger hidden sector [149]. For example, DM could be charged under a hidden U(1) gauge symmetry which could lead to strong, velocity-dependent scattering. DM could be in the form of dark atoms in such a scenario [150], for which the geometric self-interaction cross section can easily be very large [151].

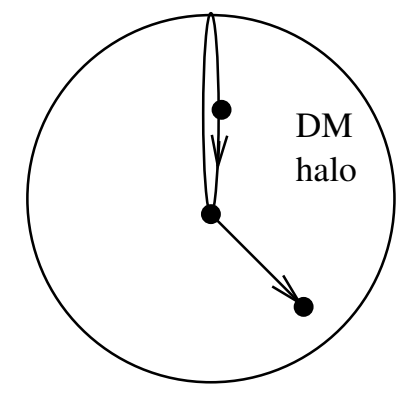

Figure 29: Coring of a cuspy halo by DM self-interactions.

In one class of models, the self-interactions can be important for determining the relic density: Strongly Interacting Massive Particle (SIMP) DM is a scenario where $\chi \chi \rightarrow f \bar{f}$ is absent or subdominant, and instead one has strong $\chi \chi \chi \rightarrow \chi \chi$ annihilations, taking the DM to be a scalar [152]. The prototypical example is pions in a dark sector, annihilating through a Wess-Zumino-Witten interaction $[153,154]$

$$
\mathscr{L} \sim \frac{1}{f^{5}} \varepsilon_{\mu v \alpha \beta} \operatorname{tr}\left(\pi \partial^{\mu} \pi \partial^{v} \pi \partial^{\alpha} \pi \partial^{\beta} \pi\right)
$$

in the notation of chiral perturbation theory [155].

We can repeat the order-of-magnitude estimate for the relic density for $3 \rightarrow 2$ annihilations. The rate of annihilation per DM particle must now depend on the density squared,

$$
\Gamma n^{2}\left\langle\sigma_{3 \rightarrow 2} v\right\rangle=H\left(m_{\chi}\right) \sim \frac{m_{\chi}^{2}}{M_{p}}
$$

leading to

$$
n \sim \frac{m_{\chi}}{\sqrt{M_{p}\left\langle\sigma_{3 \rightarrow 2} v\right\rangle}}
$$


Notice that $\sigma_{3 \rightarrow 2}$ has different dimensions than a $2 \rightarrow 2$ scattering cross section. To define its thermal average, one should examine the collision term in the Boltzmann equation; see for example [156]. If for simplicity we imagine there is only one dimensionful scale in the dark sector, so that $\sigma_{3 \rightarrow 2} \sim 1 / m_{\chi}^{5}$, we find that $m_{\chi} \sim 1 \mathrm{GeV}$ is needed to achieve the observed relic density; see problem 7 below.

\subsection{Exercises}

1. Annihilation kinematics. In the center of mass frame for two annihilating DM particles, the velocities are $\pm p / E$ along some axis. Show that the relative velocity is $2 \sqrt{1-4 m^{2} / s}$. Generalize this to the case where the annihilating particles have unequal masses, which would be relevant for coannihilating DM scenarios. (For more about coannihilating DM, see ref. [157].)

2. Thermal averaging. Suppose the DM annihilation cross section has a resonant enhancement, so that (ignoring factors of order 1)

$$
\sigma=\frac{\lambda^{4} m_{\chi}^{2}}{\sqrt{1-4 m_{\chi}^{2} / s}\left[\left(s-m_{\phi}^{2}\right)^{2}+m_{\phi}^{2} \Gamma_{\phi}^{2}\right]}
$$

where $\phi$ is the particle exchanged in the $s$ channel. In the narrow-width approximation for the resonance, you can treat the Breit-Wigner factor as a representation of the delta function. Use this approximation to evaluate the Gondolo-Gelmini thermally averaged $\sigma v$. Then take $4 m_{\chi}^{2}=m_{\phi}^{2}-\varepsilon^{2}$, where $\varepsilon \ll m_{\chi}$, and suppose that $T=m_{\chi} / 30$ (which could represent the freezeout temperature) for evaluating the $T$-dependent functions. How does the result compare to the naive procedure of evaluating $\sigma v$ at $s=4 m_{\chi}^{2}=m_{\phi}^{2}$ ?

3. Freeze-in from above. Suppose that the DM particle $\chi$ was never in thermal equilibrium, but its initial abundance $Y_{i}$ (at some initial $x_{i}$ ) was $\gg Y_{\text {eq }}$. If $Y(x)$ remains $\gg Y_{\text {eq }}$ throughout the evolution, we can solve the Boltzmann equation ignoring the $Y_{\text {eq }}$ term.

(a) Carry this out, and show that in the regime where $Y$ changes by a large factor, its final abundance is independent of $Y_{i}$.

(b) Show that the time scale for $Y$ to reach its present value is typically much shorter than the time scale governing $Y_{\mathrm{eq}}$. Use this observation to derive a bound on the relevant parameters, from the requirement that $Y \gg Y_{\mathrm{eq}}$ at all times.

(c) A similar combination of parameters determines the relic density. If this matches observations, what constraint must $m_{\chi}$ satisfy in order for this version of freeze-in to work?

4. Primordial black holes. We will try to roughly estimate the probability of producing a PBH of a given mass from inflationary density perturbations. See astro-ph/0109404 for details.

(a) The mean-squared relative mass fluctuation $\sigma^{2}=\delta M^{2} / M^{2}$ in a region of size $R$ can be obtained from the power spectrum $P_{\delta}(k)$ for the density perturbation $\delta \rho / \rho$ using the formula

$$
\sigma_{R}^{2}=\left\langle\left(\frac{\delta M^{2}}{M^{2}}\right)_{R}\right\rangle=\left\langle\left(\frac{\int d^{3} r W_{R}(r) \delta \rho(\vec{x}+\vec{r})}{\rho \int d^{3} r W_{R}(r)}\right)^{2}\right\rangle
$$


where $V$ is some large fiducial volume averaged over, $\rho$ is the mean density, and $W_{R}$ is a window function that selects a region of size $r \lesssim R$, for example the top-hat $\Theta(R-r)$ or the Gaussian $\exp \left(-r^{2} / 2 R^{2}\right)$. We will take the Gaussian for simplicity. By Fourier transforming everything and using the definition of the power spectrum

$$
\left\langle\frac{\delta \rho_{k}}{\rho} \frac{\delta \rho_{k^{\prime}}}{\rho}\right\rangle=\delta\left(\vec{k}+\vec{k}^{\prime}\right) \frac{P_{\delta}(k)}{k^{3}}
$$

(corresponding to $\delta \rho_{k}$ being a Gaussian random variable), show that

$$
\sigma_{R}^{2}=\frac{1}{2 \pi^{2}} \int \frac{d k}{k} e^{-k^{2} R^{2}} P_{\delta}(k)
$$

Here $P_{\delta}$ is normalized so that a scale-invariant spectrum would have $P_{\delta}$ constant. However remember that $P_{\delta}$ has an extra power of $k^{4}$ since $\delta \rho_{k} / \rho \sim k^{2} \mathscr{R}_{k}(\mathscr{R}$ is the 3D curvature invariant, whose power is nearly scale-invariant). For the present crude estimate, take

$$
P_{\delta}(k) \sim A_{s}\left(\frac{k}{a H}\right)^{4}
$$

where $A_{s} \sim 10^{-9}$ is the amplitude of the scalar power spectrum (in the region of $k$ measured by the $\mathrm{CMB}$ ), and $k / a H$ is the physical wave number at the moment when the comoving scale $k$ re-entered the horizon.

(b) The relative mass fluctuation $\delta_{M}=\left(\delta M^{2} / M^{2}\right)_{R}$ in a region of size $R$ is also a Gaussian random variable, whose variance we just estimated in part (a). To form a black hole, we need a rare fluctuation from the tail of the distribution such that the fluctuation is large, $\delta_{M} \sim 1$. Show that the probability to have a fluctuation in the interval $\delta \in\left[\delta_{1}, \delta_{2}\right]$ is dominated by the lower limit,

$$
P \sim \frac{\sigma_{R}}{\sqrt{2 \pi} \delta_{1}} e^{-\delta_{1}^{2} /\left(2 \sigma_{R}^{2}\right)}
$$

If $\delta_{1} \sim 1$, a black hole will form, whose mass is of order the total mass contained in the region of size $R$, namely $M \sim \rho R^{3}$. If $R$ is taken to be a comoving scale, then we can evaluate this today, taking $k \rightarrow 1 / R$ in eq. (4.32).

Assuming the scalar power is really scale-invariant, with the COBE normalization, estimate the probability to form a black hole of mass $\sim 30 M_{\odot}$.

(c) To increase the probability, one needs to assume that the scalar power spectrum is much larger at the wave numbers $k \sim 1 / R$ of interest. How large must it be to get a probability of order 1 to form a $\sim 30 M_{\odot}$ black hole?

(d) In problem 5 of set 1, you estimated the boost in power coming from a step in the inflaton potential. Translate your result from part (c) to estimate how large the step in the potential must be.

5. Misalignment mechanism. Derive the result for the relic density of an axion-like particle given in the lectures,

$$
\Omega_{a} \sim 0.3\left(\frac{m}{10^{-22} \mathrm{eV}}\right)^{1 / 2}\left(\frac{f}{4 \times 10^{16} \mathrm{GeV}}\right)^{2}
$$

6. Self-interacting dark matter. Suppose dark matter is a scalar particle with self-interaction $\lambda \phi^{4}$ and $\lambda \sim 1$. Find the mass that corresponds to a cross section such that $\sigma / m \sim 0.1 \mathrm{~cm}^{2} / \mathrm{g}$. 
7. SIMP dark matter. Consider scalar DM with mass $m$ and a $3 \rightarrow 2$ cross section of order $\sigma v \sim 1 / m^{5}$. Estimate the mass that gives the right relic density.

Acknowledgments. I thank the students of TASI for perceptive comments and questions that allowed me to improve these lectures. Thanks to the following people for helpful discussions or correspondence: H. Baer, R. Brandenberger, T. Bringmann, T. Degrand, J. Feng, W. Fischler, J. Garcia-Bellido, K. Kainulainen, R. Kolb, A. Liddle, D. Lyth, G. Moore, P. Scott, X. Tata. I especially thank M. Puel for pointing out numerous mistakes and typos. This work was supported by the Natural Sciences and Engineering Research Council (NSERC) of Canada.

\section{A. TASI-recommended road bike rides}

A useful bike map for the Boulder area can be found here.

The Boulder Creek bike path heading west is a good warm-up, though not going very far before becoming hard-packed dirt and then ending. At this writing, Fourmile Canyon Road is closed to cyclists due to reconstruction. Boulder Canyon Drive is not recommended, having heavy traffic, no shoulder, and a tunnel. A longer ride can be found by taking the sign for Settler's Park and heading toward 4th St., a designated bikeway. It ends at Linden Dr., which provides a 7.3\% climb for masochists, with little reward on the descent since much braking is required.

For a longer ride, Hygiene is a popular destination. Avoid Highway 119 even though the shoulder is wide enough; too much traffic. Boulder Creek path to Pearl, then 61st is much better. A fine loop that includes Hygiene is along US 36, turning at Hygiene Road and returning to Boulder on 65 th/63rd. The route north offers interesting possibilities for variations that require much more climbing, mainly Olde Stage Road, with a quite gratifying descent along Lefthand Canyon Drive, back to US 36 near Altona.

A more ambitious variation is Lee Hill Drive, connecting to Lefthand Canyon Drive. The latter is recommended for a longer foray into the mountains as it is quite smooth and has little traffic. (At this writing, James Canyon Dr. is still damaged by the flooding and becomes dirt at some point.) From the dorms, take Folsom/26th to Tamarack, Spotswood, 22nd, Upland, 19th, and catch the bike trail after crossing Violet; it brings you to Lee Hill Dr.

The climb up to NCAR is quite scenic, though not very long. I followed it up by continuing south out of town on the Broadway trail, which turns into Marshall Road, eventually turning into C170, a smooth highway with a good shoulder and not too much traffic. When $\mathrm{C} 170$ reaches US 36, you can cross underneath it and return via the Turnpike bike path, giving a great view of the mountains and very speedy descent. At the bottom of the descent, instead of crossing back underneath US 36 I turned right and found my way to the South Boulder Creek bike path (heading north), which was worth the trouble. It joins up with the usual Boulder Creek path heading back toward CU. Get off at Folsom for a direct path to the dorms.

\section{References}

[1] Particle Data Group collaboration, C. Patrignani et al., Review of Particle Physics, Chin. Phys. C40 (2016) 100001. 
[2] E. W. Kolb and M. S. Turner, The Early Universe, Front. Phys. 69 (1990) 1-547.

[3] M. Davis, Is the universe homogeneous on large scales?, in Critical dialogues in cosmology. Proceedings, Celebration of the 250th Anniversary of Princeton University, Princeton, USA, June 24-27, 1996, pp. 13-23, 1996, astro-ph/9610149.

[4] S. W. Hawking and E. Israel, eds., General Relativity: An Einstein Centenary Survey. Cambridge University Press, New York, NY, 1979.

[5] Ya. B. Zeldovich and M. Yu. Khlopov, On the Concentration of Relic Magnetic Monopoles in the Universe, Phys. Lett. 79B (1978) 239-241.

[6] J. Preskill, Cosmological Production of Superheavy Magnetic Monopoles, Phys. Rev. Lett. 43 (1979) 1365.

[7] A. H. Guth and S. H. H. Tye, Phase Transitions and Magnetic Monopole Production in the Very Early Universe, Phys. Rev. Lett. 44 (1980) 631.

[8] A. H. Guth, The Inflationary Universe: A Possible Solution to the Horizon and Flatness Problems, Phys. Rev. D23 (1981) 347-356.

[9] J. D. Brown and C. Teitelboim, Dynamical Neutralization of the Cosmological Constant, Phys. Lett. B195 (1987) 177-182.

[10] A. D. Linde, A New Inflationary Universe Scenario: A Possible Solution of the Horizon, Flatness, Homogeneity, Isotropy and Primordial Monopole Problems, Phys. Lett. 108B (1982) 389-393.

[11] A. Albrecht and P. J. Steinhardt, Cosmology for Grand Unified Theories with Radiatively Induced Symmetry Breaking, Phys. Rev. Lett. 48 (1982) 1220-1223.

[12] A. A. Starobinsky, A New Type of Isotropic Cosmological Models Without Singularity, Phys. Lett. B91 (1980) 99-102.

[13] Planck Collaboration collaboration, P. A. R. Ade et al., Planck 2015 results. XX. Constraints on inflation, Astron. Astrophys. 594 (2016) A20, [1502.02114].

[14] A. R. Liddle and S. M. Leach, How long before the end of inflation were observable perturbations produced?, Phys. Rev. D68 (2003) 103503, [astro-ph/ 0305263 ].

[15] S. W. Hawking, The Development of Irregularities in a Single Bubble Inflationary Universe, Phys. Lett. 115B (1982) 295.

[16] A. A. Starobinsky, Dynamics of Phase Transition in the New Inflationary Universe Scenario and Generation of Perturbations, Phys. Lett. 117B (1982) 175-178.

[17] A. H. Guth and S. Y. Pi, Fluctuations in the New Inflationary Universe, Phys. Rev. Lett. 49 (1982) 1110-1113.

[18] J. M. Bardeen, P. J. Steinhardt and M. S. Turner, Spontaneous Creation of Almost Scale - Free Density Perturbations in an Inflationary Universe, Phys. Rev. D28 (1983) 679.

[19] G. F. Smoot, C. L. Bennett, A. Kogut, E. L. Wright, J. Aymon, N. W. Boggess et al., Structure in the COBE differential microwave radiometer first-year maps, ApJ 396 (Sept., 1992) L1-L5.

[20] H. Kurki-Suonio, Lectures on Cosmological Perturbation Theory. Lecture 12: CMB; http://www.helsinki.fi/ hkurkisu /cpt/Cosmo12.pdf, .

[21] A. Lewis and S. Bridle, Cosmological parameters from CMB and other data: A Monte Carlo approach, Phys. Rev. D66 (2002) 103511, [astro-ph/ 0205436 ]. 
[22] BICEP2 collaboration, P. A. R. Ade et al., Detection of B-Mode Polarization at Degree Angular Scales by BICEP2, Phys. Rev. Lett. 112 (2014) 241101, [1403.3985].

[23] BICEP2, PLANCK collaboration, P. A. R. Ade et al., Joint Analysis of BICEP2/KeckArray and Planck Data, Phys. Rev. Lett. 114 (2015) 101301, [1502.00612].

[24] A. D. Linde, Chaotic Inflation, Phys. Lett. 129B (1983) 177-181.

[25] L. McAllister, E. Silverstein and A. Westphal, Gravity Waves and Linear Inflation from Axion Monodromy, Phys. Rev. D82 (2010) 046003, [0 808 . 0706].

[26] L. Boubekeur and D. H. Lyth, Hilltop inflation, JCAP 0507 (2005) 010, [hep-ph/ 0502047 ].

[27] J. M. Cline, String Cosmology, in Les Houches Summer School - Session 86: Particle Physics and Cosmology: The Fabric of Spacetime Les Houches, France, July 31-August 25, 2006, 2006, hep-th/ 0 612129, http://inspirehep.net/record/734399/files/arXiv:hep-th_0612129.pdf.

[28] A. R. Liddle and D. H. Lyth, The Cold dark matter density perturbation, Phys. Rept. 231 (1993) 1-105, [astro-ph/9303019].

[29] A. R. Liddle and D. H. Lyth, Cosmological inflation and large scale structure. 2000.

[30] D. H. Lyth and A. R. Liddle, The primordial density perturbation: Cosmology, inflation and the origin of structure. 2009.

[31] A. D. Linde, Inflationary Cosmology, Lect. Notes Phys. 738 (2008) 1-54, [0 705.0164$].$

[32] A. H. Guth and S.-Y. Pi, The Quantum Mechanics of the Scalar Field in the New Inflationary Universe, Phys. Rev. D32 (1985) 1899-1920.

[33] R. H. Brandenberger, Lectures on the theory of cosmological perturbations, Lect. Notes Phys. 646 (2004) 127-167, [hep-th/0306071].

[34] V. F. Mukhanov, H. A. Feldman and R. H. Brandenberger, Theory of cosmological perturbations. Part 1. Classical perturbations. Part 2. Quantum theory of perturbations. Part 3. Extensions, Phys. Rept. 215 (1992) 203-333.

[35] H. Kodama and M. Sasaki, Cosmological Perturbation Theory, Prog. Theor. Phys. Suppl. 78 (1984) $1-166$.

[36] M. Sasaki, Large Scale Quantum Fluctuations in the Inflationary Universe, Prog. Theor. Phys. 76 (1986) 1036.

[37] V. F. Mukhanov, Quantum Theory of Gauge Invariant Cosmological Perturbations, Sov. Phys. JETP 67 (1988) 1297-1302.

[38] Planck collaboration, P. A. R. Ade et al., Planck 2013 results. XXII. Constraints on inflation, Astron. Astrophys. 571 (2014) A22, [1303.5082].

[39] D. H. Lyth and D. Wands, Generating the curvature perturbation without an inflaton, Phys. Lett. B524 (2002) 5-14, [hep-ph/ 0110002$]$.

[40] E. Komatsu and D. N. Spergel, Acoustic signatures in the primary microwave background bispectrum, Phys. Rev. D63 (2001) 063002, [astro-ph / 0005036 ].

[41] N. Arkani-Hamed and J. Maldacena, Cosmological Collider Physics, 1503.08043.

[42] A. D. Dolgov and D. P. Kirilova, On particle creation by a time dependent scalar field, Sov. J. Nucl. Phys. 51 (1990) 172-177. 
[43] J. H. Traschen and R. H. Brandenberger, Particle Production During Out-of-equilibrium Phase Transitions, Phys. Rev. D42 (1990) 2491-2504.

[44] L. Kofman, A. D. Linde and A. A. Starobinsky, Reheating after inflation, Phys. Rev. Lett. 73 (1994) 3195-3198, [hep-th/9405187].

[45] L. Kofman, A. D. Linde and A. A. Starobinsky, Towards the theory of reheating after inflation, Phys. Rev. D56 (1997) 3258-3295, [hep-ph/ 9704452$].$

[46] F. Kahlhoefer and J. McDonald, WIMP Dark Matter and Unitarity-Conserving Inflation via a Gauge Singlet Scalar, JCAP 1511 (2015) 015, [1507.03600].

[47] L. H. Ford, Gravitational Particle Creation and Inflation, Phys. Rev. D35 (1987) 2955.

[48] F. L. Bezrukov and M. Shaposhnikov, The Standard Model Higgs boson as the inflaton, Phys. Lett. B659 (2008) 703-706, [0 710 . 3755].

[49] A. Linde, M. Noorbala and A. Westphal, Observational consequences of chaotic inflation with nonminimal coupling to gravity, JCAP 1103 (2011) 013, [1101.2652].

[50] A. De Felice and S. Tsujikawa, $f(R)$ theories, Living Rev. Rel. 13 (2010) 3, [1 002 . 4928 ].

[51] A. D. Sakharov, Violation of CP Invariance, $C$ asymmetry, and baryon asymmetry of the universe, Pisma Zh. Eksp. Teor. Fiz. 5 (1967) 32-35.

[52] A. Yu. Ignatiev, N. V. Krasnikov, V. A. Kuzmin and A. N. Tavkhelidze, Universal CP Noninvariant Superweak Interaction and Baryon Asymmetry of the Universe, Phys. Lett. 76B (1978) 436-438.

[53] M. Yoshimura, Unified Gauge Theories and the Baryon Number of the Universe, Phys. Rev. Lett. 41 (1978) 281-284.

[54] D. Toussaint, S. B. Treiman, F. Wilczek and A. Zee, Matter - Antimatter Accounting, Thermodynamics, and Black Hole Radiation, Phys. Rev. D19 (1979) 1036-1045.

[55] G. 't Hooft, Symmetry Breaking Through Bell-Jackiw Anomalies, Phys. Rev. Lett. 37 (1976) 8-11.

[56] V. A. Kuzmin, V. A. Rubakov and M. E. Shaposhnikov, On the Anomalous Electroweak Baryon Number Nonconservation in the Early Universe, Phys. Lett. 155B (1985) 36.

[57] J. M. Cline, Baryogenesis, in Les Houches Summer School - Session 86: Particle Physics and Cosmology: The Fabric of Spacetime Les Houches, France, July 31-August 25, 2006, 2006, hep-ph/0609145.

[58] D. Bodeker, G. D. Moore and K. Rummukainen, Chern-Simons number diffusion and hard thermal loops on the lattice, Phys. Rev. D61 (2000) 056003, [hep-ph/9907545].

[59] D. V. Nanopoulos and S. Weinberg, Mechanisms for Cosmological Baryon Production, Phys. Rev. D20 (1979) 2484.

[60] R. E. Cutkosky, Singularities and discontinuities of Feynman amplitudes, J. Math. Phys. 1 (1960) 429-433.

[61] W. Buchmuller, P. Di Bari and M. Plumacher, Leptogenesis for pedestrians, Annals Phys. 315 (2005) 305-351, [hep-ph/0401240].

[62] M.-C. Chen, TASI 2006 Lectures on Leptogenesis, in Proceedings of Theoretical Advanced Study Institute in Elementary Particle Physics : Exploring New Frontiers Using Colliders and Neutrinos (TASI 2006): Boulder, Colorado, June 4-30, 2006, pp. 123-176, 2007, hep-ph / 0703087 , http://lss.fnal.gov/cgi-bin/find_paper.pl?pub-07-059. 
[63] S. Davidson and A. Ibarra, A Lower bound on the right-handed neutrino mass from leptogenesis, Phys. Lett. B535 (2002) 25-32, [hep-ph/ 0202239$].$

[64] R. N. Mohapatra and J. W. F. Valle, Neutrino Mass and Baryon Number Nonconservation in Superstring Models, Phys. Rev. D34 (1986) 1642.

[65] S. Blanchet, P. S. B. Dev and R. N. Mohapatra, Leptogenesis with TeV Scale Inverse Seesaw in SO(10), Phys. Rev. D82 (2010) 115025, [1010.1471].

[66] A. Pilaftsis and T. E. J. Underwood, Resonant leptogenesis, Nucl. Phys. B692 (2004) 303-345, [hep-ph/0309342].

[67] A. Pilaftsis and T. E. J. Underwood, Electroweak-scale resonant leptogenesis, Phys. Rev. D72 (2005) 113001, [hep-ph/0506107].

[68] G. R. Farrar and M. E. Shaposhnikov, Baryon asymmetry of the universe in the minimal Standard Model, Phys. Rev. Lett. 70 (1993) 2833-2836, [hep-ph/9305274].

[69] G. R. Farrar and M. E. Shaposhnikov, Baryon asymmetry of the universe in the standard electroweak theory, Phys. Rev. D50 (1994) 774, [hep-ph/9305275].

[70] M. B. Gavela, P. Hernandez, J. Orloff and O. Pene, Standard model CP violation and baryon asymmetry, Mod. Phys. Lett. A9 (1994) 795-810, [hep-ph/9312215].

[71] A. G. Cohen, D. B. Kaplan and A. E. Nelson, Baryogenesis at the weak phase transition, Nucl. Phys. B349 (1991) 727-742.

[72] A. G. Cohen, D. B. Kaplan and A. E. Nelson, Weak scale baryogenesis, Phys. Lett. B245 (1990) $561-564$.

[73] M. Joyce, T. Prokopec and N. Turok, Electroweak baryogenesis from a classical force, Phys. Rev. Lett. 75 (1995) 1695-1698, [hep-ph/9408339].

[74] M. Joyce, T. Prokopec and N. Turok, Nonlocal electroweak baryogenesis. Part 2: The Classical regime, Phys. Rev. D53 (1996) 2958-2980, [hep-ph/9410282].

[75] J. M. Cline, M. Joyce and K. Kainulainen, Supersymmetric electroweak baryogenesis, JHEP 07 (2000) 018, [hep-ph/0006119].

[76] L. Fromme and S. J. Huber, Top transport in electroweak baryogenesis, JHEP 03 (2007) 049, [hep-ph/0604159].

[77] S. Yu. Khlebnikov and M. E. Shaposhnikov, The Statistical Theory of Anomalous Fermion Number Nonconservation, Nucl. Phys. B308 (1988) 885-912.

[78] G. D. Moore, A Nonperturbative measurement of the broken phase sphaleron rate, Phys. Lett. B439 (1998) 357-365, [hep-ph/9801204].

[79] J. Garcia-Bellido, D. Yu. Grigoriev, A. Kusenko and M. E. Shaposhnikov, Nonequilibrium electroweak baryogenesis from preheating after inflation, Phys. Rev. D60 (1999) 123504, [hep-ph/9902449].

[80] G. D. Moore, Measuring the broken phase sphaleron rate nonperturbatively, Phys. Rev. D59 (1999) 014503, [hep-ph/9805264].

[81] A. D. Linde, On the Vacuum Instability and the Higgs Meson Mass, Phys. Lett. 70B (1977) 306-308.

[82] A. D. Linde, Decay of the False Vacuum at Finite Temperature, Nucl. Phys. B216 (1983) 421. 
[83] G. W. Anderson and L. J. Hall, The Electroweak phase transition and baryogenesis, Phys. Rev. D45 (1992) 2685-2698.

[84] K. Rummukainen, M. Tsypin, K. Kajantie, M. Laine and M. E. Shaposhnikov, The Universality class of the electroweak theory, Nucl. Phys. B532 (1998) 283-314, [hep-lat/9805013].

[85] J. R. Espinosa, T. Konstandin and F. Riva, Strong Electroweak Phase Transitions in the Standard Model with a Singlet, Nucl. Phys. B854 (2012) 592-630, [1107. 5441].

[86] C. Caprini et al., Science with the space-based interferometer eLISA. II: Gravitational waves from cosmological phase transitions, JCAP 1604 (2016) 001, [1512. 06239 ].

[87] P. Huang, A. J. Long and L.-T. Wang, Probing the Electroweak Phase Transition with Higgs Factories and Gravitational Waves, Phys. Rev. D94 (2016) 075008, [1608. 06619 ].

[88] G. D. Moore and T. Prokopec, How fast can the wall move? A Study of the electroweak phase transition dynamics, Phys. Rev. D52 (1995) 7182-7204, [hep-ph/ 9506475$].$

[89] P. John and M. G. Schmidt, Do stops slow down electroweak bubble walls?, Nucl. Phys. B598 (2001) 291-305, [hep-ph/ 0002050$]$.

[90] I. Affleck and M. Dine, A New Mechanism for Baryogenesis, Nucl. Phys. B249 (1985) 361-380.

[91] F. Zwicky, Die Rotverschiebung von extragalaktischen Nebeln, Helv. Phys. Acta 6 (1933) 110-127.

[92] G. Bertone and D. Hooper, A History of Dark Matter, Submitted to: Rev. Mod. Phys. (2016), [1605.04909].

[93] J. H. Oort, The force exerted by the stellar system in the direction perpendicular to the galactic plane and some related problems, Bull. Astron. Inst. Netherlands 6 (Aug., 1932) 249.

[94] F. Zwicky, On the Masses of Nebulae and of Clusters of Nebulae, ApJ 86 (Oct., 1937) 217.

[95] S. M. Faber and J. S. Gallagher, Masses and mass-to-light ratios of galaxies, Ann. Rev. Astron. Astrophys. 17 (1979) 135-183.

[96] V. C. Rubin and W. K. Ford, Jr., Rotation of the Andromeda Nebula from a Spectroscopic Survey of Emission Regions, ApJ 159 (Feb., 1970) 379.

[97] H. W. Babcock, The rotation of the Andromeda Nebula, Lick Observatory Bulletin 19 (1939) 41-51.

[98] A. De Rujula and S. L. Glashow, Galactic Neutrinos and UV Astronomy, Phys. Rev. Lett. 45 (1980) 942.

[99] S. D. M. White, C. S. Frenk and M. Davis, Is the universe made of massive neutrinos?, in Formation and evolution of galaxies and large structures in the universe. Proceedings, NATO Advanced Study Institute, 3rd Moriond astrophysics meeting, La Plagne, France, March, 1983, 1984.

[100] G. Steigman and M. S. Turner, Cosmological Constraints on the Properties of Weakly Interacting Massive Particles, Nucl. Phys. B253 (1985) 375-386.

[101] B. W. Lee and S. Weinberg, Cosmological Lower Bound on Heavy Neutrino Masses, Phys. Rev. Lett. 39 (1977) 165-168.

[102] A. Drukier and L. Stodolsky, Principles and Applications of a Neutral Current Detector for Neutrino Physics and Astronomy, Phys. Rev. D30 (1984) 2295.

[103] M. W. Goodman and E. Witten, Detectability of Certain Dark Matter Candidates, Phys. Rev. D31 (1985) 3059. 
[104] M. Viel, J. Lesgourgues, M. G. Haehnelt, S. Matarrese and A. Riotto, Constraining warm dark matter candidates including sterile neutrinos and light gravitinos with WMAP and the Lyman-alpha forest, Phys. Rev. D71 (2005) 063534, [astro-ph/0501562].

[105] J. F. Navarro, C. S. Frenk and S. D. M. White, A Universal density profile from hierarchical clustering, Astrophys. J. 490 (1997) 493-508, [astro-ph/9611107].

[106] D. H. Weinberg, J. S. Bullock, F. Governato, R. Kuzio de Naray and A. H. G. Peter, Cold dark matter: controversies on small scales, Proc. Nat. Acad. Sci. 112 (2015) 12249-12255, [1306.0913].

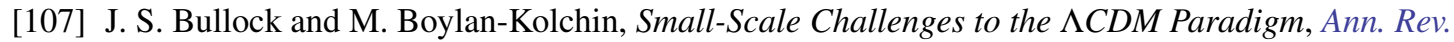
Astron. Astrophys. 55 (2017) 343-387, [1707.04256].

[108] V. Iršič et al., New Constraints on the free-streaming of warm dark matter from intermediate and small scale Lyman- $\alpha$ forest data, Phys. Rev. D96 (2017) 023522, [1702 . 01764 ].

[109] G. Jungman, M. Kamionkowski and K. Griest, Supersymmetric dark matter, Phys. Rept. 267 (1996) 195-373, [hep-ph/9506380].

[110] H. Baer and X. Tata, Dark matter and the LHC, in Physics at the Large Hadron Collider (A. Datta, B. Mukhopadhyaya, A. Raychaudhuri, A. K. Gupta, C. L. Khetrapal, T. Padmanabhan et al., eds.), pp. 179-203. 2009. 0805 .1905. DOI.

[111] M. Cannoni, Relativistic $\left\langle\sigma v_{\text {rel }}>\right.$ in the calculation of relics abundances: a closer look, Phys. Rev. D89 (2014) 103533, [1311.4494].

[112] P. Gondolo and G. Gelmini, Cosmic abundances of stable particles: Improved analysis, Nucl. Phys. B360 (1991) 145-179.

[113] G. Steigman, B. Dasgupta and J. F. Beacom, Precise Relic WIMP Abundance and its Impact on Searches for Dark Matter Annihilation, Phys. Rev. D86 (2012) 023506, [1204 . 3622].

[114] G. Belanger, F. Boudjema, A. Pukhov and A. Semenov, micrOMEGAs_3: A program for calculating dark matter observables, Comput. Phys. Commun. 185 (2014) 960-985, [1305 . 0237].

[115] T. Bringmann, J. Edsjö, P. Gondolo, P. Ullio and L. Bergström, DarkSUSY 6 : An Advanced Tool to Compute Dark Matter Properties Numerically, 1802.03399.

[116] R. J. Scherrer and M. S. Turner, On the Relic, Cosmic Abundance of Stable Weakly Interacting Massive Particles, Phys. Rev. D33 (1986) 1585.

[117] J. M. Cline, K. Kainulainen, P. Scott and C. Weniger, Update on scalar singlet dark matter, Phys. Rev. D88 (2013) 055025, [1306.4710].

[118] K. Griest and M. Kamionkowski, Unitarity Limits on the Mass and Radius of Dark Matter Particles, Phys. Rev. Lett. 64 (1990) 615.

[119] K. Harigaya, M. Ibe, K. Kaneta, W. Nakano and M. Suzuki, Thermal Relic Dark Matter Beyond the Unitarity Limit, JHEP 08 (2016) 151, [1606.00159].

[120] K. M. Zurek, Asymmetric Dark Matter: Theories, Signatures, and Constraints, Phys. Rept. 537 (2014) 91-121, [1308.0338].

[121] M. L. Graesser, I. M. Shoemaker and L. Vecchi, Asymmetric WIMP dark matter, JHEP 10 (2011) $110,[1103.2771]$.

[122] T. Lin, H.-B. Yu and K. M. Zurek, On Symmetric and Asymmetric Light Dark Matter, Phys. Rev. D85 (2012) 063503, [1111.0293]. 
[123] M. Garny, M. Sandora and M. S. Sloth, Planckian Interacting Massive Particles as Dark Matter, Phys. Rev. Lett. 116 (2016) 101302, [1511.03278].

[124] M. Garny, A. Palessandro, M. Sandora and M. S. Sloth, Theory and Phenomenology of Planckian Interacting Massive Particles as Dark Matter, JCAP 1802 (2018) 027, [1709. 09688].

[125] T. Banks and N. Seiberg, Symmetries and Strings in Field Theory and Gravity, Phys. Rev. D83 (2011) 084019, [1011.5120].

[126] B. Carr, F. Kuhnel and M. Sandstad, Primordial Black Holes as Dark Matter, Phys. Rev. D94 (2016) 083504, [1607.06077].

[127] VIRGO, LIGO SCIENTIFIC collaboration, B. P. Abbott et al., Observation of Gravitational Waves from a Binary Black Hole Merger, Phys. Rev. Lett. 116 (2016) 061102, [1602 . 03837 ].

[128] M. Ricotti, J. P. Ostriker and K. J. Mack, Effect of Primordial Black Holes on the Cosmic Microwave Background and Cosmological Parameter Estimates, Astrophys. J. 680 (2008) 829, [0 709.0524 ].

[129] Y. Ali-Haïmoud and M. Kamionkowski, Cosmic microwave background limits on accreting primordial black holes, Phys. Rev. D95 (2017) 043534, [1612. 0564 4].

[130] T. D. Brandt, Constraints on MACHO Dark Matter from Compact Stellar Systems in Ultra-Faint Dwarf Galaxies, Astrophys. J. 824 (2016) L31, [1605. 03665$].$

[131] J. Garcia-Bellido, A. D. Linde and D. Wands, Density perturbations and black hole formation in hybrid inflation, Phys. Rev. D54 (1996) 6040-6058, [astro-ph/ 9605094 ].

[132] T. Bringmann, C. Kiefer and D. Polarski, Primordial black holes from inflationary models with and without broken scale invariance, Phys. Rev. D65 (2002) 024008, [a stro-ph/ 0109404 ].

[133] A. A. Starobinsky, Spectrum of adiabatic perturbations in the universe when there are singularities in the inflation potential, JETP Lett. 55 (1992) 489-494.

[134] T. Bringmann, P. Scott and Y. Akrami, Improved constraints on the primordial power spectrum at small scales from ultracompact minihalos, Phys. Rev. D85 (2012) 125027, [1110 . 2484 ].

[135] A. M. Green, A. R. Liddle, K. A. Malik and M. Sasaki, A New calculation of the mass fraction of primordial black holes, Phys. Rev. D70 (2004) 041502, [astro-ph / 0403181 ].

[136] L. F. Abbott and P. Sikivie, A Cosmological Bound on the Invisible Axion, Phys. Lett. B120 (1983) 133-136.

[137] M. Dine and W. Fischler, The Not So Harmless Axion, Phys. Lett. B120 (1983) 137-141.

[138] P. W. Graham, I. G. Irastorza, S. K. Lamoreaux, A. Lindner and K. A. van Bibber, Experimental Searches for the Axion and Axion-Like Particles, Ann. Rev. Nucl. Part. Sci. 65 (2015) 485-514, [1602.00039].

[139] L. Hui, J. P. Ostriker, S. Tremaine and E. Witten, Ultralight scalars as cosmological dark matter, Phys. Rev. D95 (2017) 043541, [1610.08297].

[140] W. Hu, R. Barkana and A. Gruzinov, Cold and fuzzy dark matter, Phys. Rev. Lett. 85 (2000) 1158-1161, [astro-ph/0003365].

[141] J. Preskill, M. B. Wise and F. Wilczek, Cosmology of the Invisible Axion, Phys. Lett. B120 (1983) 127-132.

[142] V. B. Klaer and G. D. Moore, The dark-matter axion mass, JCAP 1711 (2017) 049, [1708 . 07521$].$ 
[143] D. N. Spergel and P. J. Steinhardt, Observational evidence for selfinteracting cold dark matter, Phys. Rev. Lett. 84 (2000) 3760-3763, [astro-ph/9909386].

[144] S. Tulin and H.-B. Yu, Dark Matter Self-interactions and Small Scale Structure, Phys. Rept. 730 (2018) 1-57, [1705.02358].

[145] E. Bertschinger, Self-similar secondary infall and accretion in an Einstein-de Sitter universe, ApJS 58 (May, 1985) 39-65.

[146] M. Vogelsberger, J. Zavala and A. Loeb, Subhaloes in Self-Interacting Galactic Dark Matter Haloes, Mon. Not. Roy. Astron. Soc. 423 (2012) 3740, [1201. 5892].

[147] M. Rocha, A. H. G. Peter, J. S. Bullock, M. Kaplinghat, S. Garrison-Kimmel, J. Onorbe et al., Cosmological Simulations with Self-Interacting Dark Matter I: Constant Density Cores and Substructure, Mon. Not. Roy. Astron. Soc. 430 (2013) 81-104, [1208. 3025].

[148] S. W. Randall, M. Markevitch, D. Clowe, A. H. Gonzalez and M. Bradac, Constraints on the Self-Interaction Cross-Section of Dark Matter from Numerical Simulations of the Merging Galaxy Cluster 1E 0657-56, Astrophys. J. 679 (2008) 1173-1180, [0 704 .0261].

[149] N. Arkani-Hamed, D. P. Finkbeiner, T. R. Slatyer and N. Weiner, A Theory of Dark Matter, Phys. Rev. D79 (2009) 015014, [0810.0713].

[150] D. E. Kaplan, G. Z. Krnjaic, K. R. Rehermann and C. M. Wells, Atomic Dark Matter, JCAP 1005 (2010) 021, [0909.0753].

[151] J. M. Cline, Z. Liu, G. Moore and W. Xue, Scattering properties of dark atoms and molecules, Phys. Rev. D89 (2014) 043514, [1311.6468].

[152] Y. Hochberg, E. Kuflik, T. Volansky and J. G. Wacker, Mechanism for Thermal Relic Dark Matter of Strongly Interacting Massive Particles, Phys. Rev. Lett. 113 (2014) 171301, [1402 . 5143].

[153] J. Wess and B. Zumino, Consequences of anomalous Ward identities, Phys. Lett. 37B (1971) 95-97.

[154] E. Witten, Global Aspects of Current Algebra, Nucl. Phys. B223 (1983) 422-432.

[155] H. Georgi, Weak Interactions and Modern Particle Theory. 1984.

[156] E. Kuflik, M. Perelstein, N. R.-L. Lorier and Y.-D. Tsai, Phenomenology of ELDER Dark Matter, JHEP 08 (2017) 078, [1706.05381].

[157] K. Griest and D. Seckel, Three exceptions in the calculation of relic abundances, Phys. Rev. D43 (1991) 3191-3203. 